\title{
Probability of Detection as a Function of Multiple Influencing Parameters
}

\section{Dissertation}

\author{
zur Erlangung des Grades \\ Doktor der Ingenieurwissenschaften (Dr.-Ing.) \\ der Naturwissenschaftlich-Technischen Fakultät III \\ Chemie, Pharmazie, Bio- und Werkstowissenschaften \\ der Universität des Saarlandes
}

vorgelegt von
Mato Pavlović

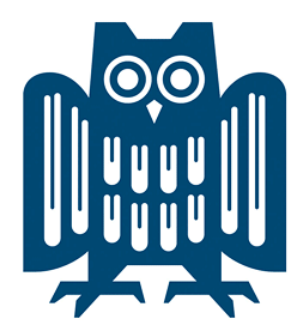

Saarbrücken 2014 
Tag des Kolloquiums:

Dekan:

Mitglieder des Prüfungsausschusses

Vorsitzender:

Gutachter:

Akademischer Mitarbeiter:
15.10.2014.

Prof. Dr. Volkhard Helms

Prof. Dr. -Ing. Christian Boller

Dir. u. Prof. Dr. -Ing. Anton Erhard

Dr. -Ing. Zhaoyu Chen 


\begin{abstract}
Non-destructive testing is subject to measurement uncertainties. In safety critical applications the reliability assessment of its capability to detect flaws is therefore necessary. In most applications, the flaw size is the single most important parameter that influences the probability of detection (POD) of the flaw. That is why the POD is typically calculated and expressed as a function of the flaw size. The capability of the inspection system to detect flaws is established by comparing the size of reliably detected flaw with the size of the flaw that is critical for the structural integrity.

Applications where several factors have an important influence on the POD are investigated in this dissertation. To devise a reliable estimation of the NDT system capability it is necessary to express the POD as a function of all these factors. A multi-parameter POD model is developed. It enables POD to be calculated and expressed as a function of several influencing parameters. The model was tested on the data from the ultrasonic inspection of copper and cast iron components with artificial flaws. Also, a technique to spatially present POD data called the volume POD is developed. The fusion of the POD data coming from multiple inspections of the same component with different sensors is performed to reach the overall POD of the inspection system.
\end{abstract}




\section{Zusammenfassung}

Zerstörungsfreie Prüfungen (ZfP) weisen Messunsicherheiten auf. Bei sicherheitsrelevanten Anwendungen der ZfP ist es notwendig, die Fähigkeit, Defekte zu entdecken, genau zu bewerten. Bei den meisten Anwendungen ist die Defektgröße der einzige Parameter, der die Entdeckungswahrscheinlichkeit (Probability of Detection - POD) des Defektes bestimmt. Deshalb wird die POD typischer Weise als eine Funktion der Defektgröße berechnet und dargestellt. Die Leistungsfähigkeit des ZfP-Systems wird ermittelt, in dem die Fehlergröße mit hinreichend großer POD verglichen wird mit der Fehlergröße, bei der die strukturelle Integrität kritisch wird.

In dieser Dissertation wurden Anwendungen untersucht, bei denen mehrere Faktoren einen wichtigen Einfluss auf die POD des Defektes haben. Um eine zuverlässige Einschätzung der Fähigkeit des ZfP-Systems zu ermöglichen ist es nötig, die POD als eine Funktion aller einflussnehmenden Parameter auszudrücken. Daher wurde das Multi-Parameter POD-Modell entwickelt. Das Modell ermöglicht die Berechnung und Darstellung der POD als eine Funktion mehrer Parameter. Das Modell wurde auf Ultraschallprüfdaten von Kupfer- und Gusseisenkomponenten angewendet. Außerdem wurde ein neuartiges Modell entwickelt, um POD-Daten darzustellen, bei denen die Position des Fehlers einen wichtigen Einfluss auf die Bewertung hat. Dieses Modell wird als Volumen-POD bezeichnet. Eine Verschmelzung der Volumen-POD-Daten wurde eingeführt, bei der die Daten von verschiedenen Prüfungen des gleichen Bauteils stammen, um eine Gesamt-POD des ZfP-Systems zu erhalten. 


\section{Acknowledgements}

The accomplishment of this thesis would not have been possible without the guidance, contribution and support from many different people. First of all, I am in great debt to the project leader Christina Müller for giving me the opportunity to work on the project and whose encouragement and guidance enabled me to develop an understanding of the subject. I am truly indebted and thankful to my colleague and friend Kazunori Takahashi for countless discussions, advices, patience, support and friendship. It is an honour for me to thank my supervisor Christian Boller. I owe sincere and earnest thankfulness to Ulf Ronneteg from SKB for his endless support and help with experiments. I would like to show my gratitude to Jorma Pitkänen from Posiva Oy for the inspiration and wisdom he extended. I would like to thank Vladimir Spokoiny from WIAS for the statistical insights and advices he shared. I am obliged to Rainer Boehm from BAM for assistance and support in simulation. I am also indebted to many of my colleagues in BAM for fruitful discussions and support, in the first place Christian Golwitzer, Daniel Kanzler and Gerd-Rüdiger Jaenisch. I would like to show my gratitude to the head of the department Uwe Ewert for his unreserved support. I am grateful to Anton Erhard for sharing his expertise in discussions that made the thesis more concise. Finally, I would like to thank my family and friends for their love and patience. 


\section{Contents}

$\begin{array}{ll}\text { List of Figures } & \mathbf{v}\end{array}$

List of Tables vii

Glossary ix

1 Introduction 1

1.1 Background of the study . . . . . . . . . . . . . 2

1.1 .1 Engineering materials $\ldots \ldots \ldots \ldots$. . . . . . . . . .

1.1.2 Structural integrity . . . . . . . . . . . . . 5

1.1.3 Non-destructive testing . . . . . . . . . . . 6 6

1.1.4 Nuclear waste management . . . . . . . . . . . . . . . . 14

1.2 Organisation of the thesis $\ldots \ldots \ldots \ldots \ldots$

2 State-of-the-Art in Reliability of Non-destructive Testing Systems 25

2.1 Definition of reliability . . . . . . . . . . . . . 26 26

2.2 Modular model . . . . . . . . . . . . . . . . . . . . . 26

2.3 Signal detection . . . . . . . . . . . . . . . . . . . . . . . . . . . 28 28

2.4 Receiver operating characteristic (ROC) . . . . . . . . . . . . . 30

2.5 Probability of detection (POD) curve . . . . . . . . . . . . . 33

2.6 Computer simulation in NDT . . . . . . . . . . . . . 41

3 Problem Statement 43

3.1 Influence of multiple parameters on the flaw severity and on the POD . 44

3.2 Ultrasonic attenuation and its influence on the POD . . . . . . . . 46

3.3 Multiple inspections of a component and an overall POD . . . . . . 48 
4 Study I 51

4.1 Influence of flaw length and flaw depth extension on the POD . . . . . 51

4.1.1 Materials and methods . . . . . . . . . . . . . . . .51

$4.1 .2 \quad$ Experimental set-up . . . . . . . . . . . . . . . . 54

4.1.3 Results and Discussion . . . . . . . . . . . . . . 56

4.2 Influence of ultrasonic attenuation on the POD . . . . . . . . . . 64

4.2.1 Materials and methods . . . . . . . . . . . . . . 64

4.2 .2 Experimental set-up . . . . . . . . . . . . . . . 66 66

4.2 .3 Results and discussion . . . . . . . . . . . . . . . 70

5 Study II

5.1 Volume POD and POD data fusion . . . . . . . . . . . . . 755

5.1 .1 Materials and methods . . . . . . . . . . . . . . 775

5.1 .2 Experimental set-up . . . . . . . . . . . . . . 76

5.1 .3 Results and discussion . . . . . . . . . . . . . . . . 79

6 Conclusions 85

6.1 Summary of contributions . . . . . . . . . . . . . . 85

6.1.1 Multi-parameter POD model . . . . . . . . . . . . . . 85

6.1.2 The POD expressed as a function of the attenuation coefficient . 87

6.1.3 Spatial representation of POD data and a POD data fusion . . . 88

6.2 Recommendations for future work . . . . . . . . . . . . 89

6.2.1 Application of the multi-parameter model on the real flaws . . . 89

6.2.2 Replacement of the maximum amplitude with a more comprehensive response signal characteristic . . . . . . . . . . . . 90 90

6.2.3 Application of the multi-parameter model on other NDT methods 91

A Copper specimens

B Cast iron specimen

References 


\section{List of Figures}

1.1 Angled dual transducer $\ldots \ldots \ldots$. . . . . . . . . . . . . . 9

1.2 Ultrasonic wave amplitude on the material boundary . . . . . . . . . . 10

1.3 Ultrasonic focused beam . . . . . . . . . . . . . . . . . . . . . . 11

1.4 Overall change of amplitude . . . . . . . . . . . . . . 13

1.5 KBS-3 method . . . . . . . . . . . . . . . . . 16

1.6 Barriers in the final repository $\ldots \ldots \ldots \ldots \ldots$

1.7 Exploded view of the canister . . . . . . . . . . . . . 18

1.8 Different versions of the insert . . . . . . . . . . . . . . . . 19

2.1 Modular model . . . . . . . . . . . . . . . . . . . 27 27

2.2 Signal detection in the ultrasonic A-scan . . . . . . . . . . . . . 29

2.3 Signal and noise probability distributions . . . . . . . . . . . 31

2.4 Receiver operating characteristics (ROC) curve . . . . . . . . . . 32

2.5 Comparison of ROC curves . . . . . . . . . . . . . . 33

$2.6 \hat{a}-a \operatorname{diagram} \ldots \ldots \ldots \ldots$

2.7 Probability of detection (POD) curve . . . . . . . . . . . 37

2.8 Comparison of $\hat{a}-a$ diagrams . . . . . . . . . . . . 39

2.9 Comparison of POD curves . . . . . . . . . . . . . . . 40

3.1 Semi-elliptical, surface breaking, crack-like flaw . . . . . . . . . 45

3.2 Location of $\max$ principal stress . . . . . . . . . . . . . . . . 45

3.3 C-scan of the whole tube . . . . . . . . . . . . . . 4 47

3.4 Preliminary plan of lid inspections . . . . . . . . . . . . . . 4 49

4.1 Installation with TRL probes . . . . . . . . . . . . . 555

$4.2 \quad$ Flaws geometry . . . . . . . . . . . . . . . . . . 55 


\section{LIST OF FIGURES}

4.3 Simulation of the TRL sound field . . . . . . . . . . . . 57

4.4 Conventional $\hat{a}$ versus $a$ diagram $\ldots \ldots \ldots \ldots \ldots$

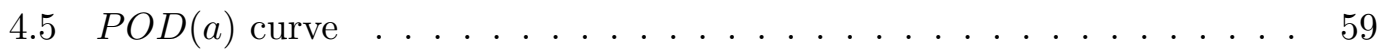

4.6 Simulated signal response . . . . . . . . . . . . . . . . 59

4.7 Multi-parameter $\hat{a}-a_{M P}$ diagram $\ldots \ldots \ldots \ldots$

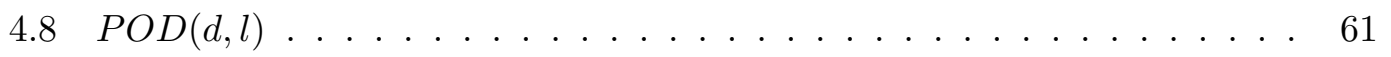

$4.9 P O D(d, l=$ const $)$ and $P O D(d=$ const,$l) \ldots \ldots \ldots 2$

4.10 Comparison of the conventional and multi-parameter POD . . . . . 6 63

4.11 Copper specimen . . . . . . . . . . . . . . . . . . 66 66

4.12 C-scan . . . . . . . . . . . . . . . . . 6 67

4.13 A-scan . . . . . . . . . . . . . . . . . 67 67

4.14 Ultrasonic wave amplitude in the specimen with plan-parallel surfaces . 68

4.15 Multiple back-wall echo amplitudes . . . . . . . . . . . . . 69

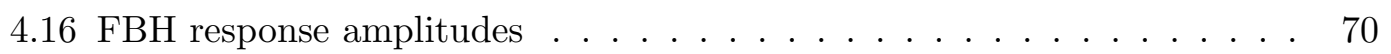

$4.17 \hat{a}-a$ diagram . . . . . . . . . . . . . . . . 71

4.18 POD curves with lower $95 \%$ confidence bounds for constant $d \ldots \ldots$

4.19 POD curves with lower $95 \%$ confidence bounds for constant $\alpha \ldots \ldots 73$

5.1 Rotating water tank . . . . . . . . . . . . 77

5.2 Transducer positioning for the lid inspection . . . . . . . . . . 78

5.3 POD as a function of depth and angle for constant diameter . . . . . . 80

5.4 POD as a function of depth and diameter for constant angle . . . . . . 81

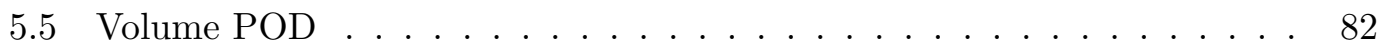

5.6 Acceptance criteria . . . . . . . . . . . . . . . . . 83

A.1 Copper specimen BAM1 . . . . . . . . . . . . . . . 94

A.2 Copper specimen BAM1B . . . . . . . . . . . . . . . 95

B.1 Cast iron specimen KLM162, section A-A . . . . . . . . . . 106

B.2 Cast iron specimen KLM162, section B-B . . . . . . . . . . . . . 107 


\section{List of Tables}

1.1 Characteristics of ultrasonic testing . . . . . . . . . . 8

1.2 Electricity production from nuclear energy . . . . . . . . . . . . . 14

1.3 Possible flaws in the cast iron insert . . . . . . . . . . . . . 21

1.4 Possible flaws in the copper tube ................ 22

2.1 Matrix of stimuli and responses in two-alternate decision task . . . . . 30

3.1 Acceptable semi-elliptical crack dimensions located in the zone of maximum principal stress for a $5 \mathrm{~cm}$ shear . . . . . . . . 46 46

A.1 Flat-bottom holes identification list . . . . . . . . . . . 96

A.2 Response signal amplitudes, phased array . . . . . . . . . . . . 100

B.1 Response signal amplitudes, TRL . . . . . . . . . . . . . . 108 
Discontinuity An intentional or unintentional interruption in the physical structure or configuration of a material or component

False Alarm Same as False positive

\section{Glossary}

A-scan A method of ultrasonic data presentation utilizing a horizontal baseline, that indicates distance, and a vertical deflection from the baseline, that indicates amplitude

Amplitude A vertical height of a signal, usually base to peak, e.g. when indicated by an A-scan presentation

Artificial flaw A flaw which has been artificially manufactured in the component, usually of very simple geometry (e.g. $\mathrm{FBH}$ or $\mathrm{SDH})$

B-scan A method of ultrasonic data presentation that displays a profile (crosssectional) view of the test specimen

C-scan A method of ultrasonic data presentation that displays a plan-type view of the test specimen

Canister A corrosion-resistant container used to enclose high-level nuclear waste during long-term storage

Correct rejection Same as True negative

Crack A clean (crystalline) fracture passing through or across the grain boundaries

Defect One or more flaws whose aggregate size, shape, orientation, location, or properties do not meet specified acceptance criteria and are rejectable
False Negative An erroneous reporting of a flaw-free part from a part of a test object which, in fact, contains flaws

False Positive An erroneous reporting of a flaw indication from a part of a test object which is, in fact, free of flaws

FBH Flat bottom hole, a kind of artificial flaw drilled in the test object with circular, flat bottom

Flaw A discontinuity that may be detectable by NDT and is not necessarily rejectable

Hit Same as True positive

HLW High-level waste; radioactive waste material, such as spent nuclear fuel initially having a high activity and thus needing constant cooling for several decades before it can be reprocessed or treated

ILW Intermedate-level waste; radioactive waste material, such as reactor and processing-plant components

Indication A response or evidence from a NDT system

KBS-3 Technology for disposal of high-level radioactive waste developed in Sweden

LLW Low-level waste; waste material contaminated by traces of radioactivity

Miss Same as False negative

NDE Non-destructive evaluation; another term for NDT

NDI Non-destructive inspection; another term for NDT 
NDT Non-destructive Testing; the development and application of technical methods to examine materials or components in ways that do not impair future usefulness and serviceability in order to detect, locate, measure and evaluate flaws; to assess integrity, properties and composition; and to measure geometrical characteristics

POD

Probability of Detection; a fraction of flaws of a nominal size that are expected to be detected

Real flaw A flaw which has developed in a component during its manufacture or in service, without any steps having been taken to deliberately encourage its development

Realistic flaw A flaw deliberately introduced into a test piece which simulates the metallurgical appearance of a real flaw. The most useful types of realistic flaw for qualification purposes are those whose NDT responses resemble, or can be related to, those of the real flaws of interest, for the inspection techniques being considered

ROC Receiver operating characteristic; a graphical plot of the $\mathrm{P}(\mathrm{TP})-\mathrm{P}(\mathrm{FP})$ at various threshold settings

SDH Side drilled hole, a kind of artificial flaw drilled in a test object

SNR Signal to noise, ratio of signal and noise

TRL Transmitter-receiver longitudinal; an ultrasonic technique using dual element, angle beam probes generating longitudinal waves

True negative Correct reporting of a flaw-free part from a part of a test object which is, indeed, free of flaws

True positive Correct reporting of a flaw indication from a part of a test object which, indeed, contains a flaw 


\section{1}

\section{Introduction}

The importance of engineering materials is best illustrated by the fact that different periods of our civilisation were named after the material that was prevalently used at the time. In a constant endeavour to create structures that are more safe and reliable, the knowledge of material properties and operating environment has constantly expanded [1]. The understanding of the relationship between the micro-structure and properties of materials enabled the design of sophisticated engineering structures. However, the properties of ideally homogeneous material are greatly degraded when discontinuities are present in a material. The existence of discontinuities in the engineering materials is tolerated if corrective actions are taken before they become critical for structural integrity. This can only function if an adequate inspection system is available - a system that is able to detect discontinuities before they become critical. Nondestructive testing is an examination of materials in ways that do not impair future usefulness [2]. Components are examined to determine presence of flaws in them, so that corrective measures can be carried out timely. Therefore, the capability of inspection systems to detect flaws needs to be quantified. Because the inspection process is subject to the inevitable uncertainty that attends all measurements, in applications where loss of structural integrity could lead to catastrophic consequences, the capability of detecting flaws is assessed in terms of reliability.

The reliability of a NDT system is defined as a degree that the NDT system is capable of achieving its purpose regarding detection, characterization and false calls where the NDT system consists of procedures, equipment and personnel that are used in performing the inspection [3]. The probability of detection (POD) curve is considered 


\section{INTRODUCTION}

to be a standard method for quantitative system capabilities assessment [4]. In a conventional, signal response analysis, the POD is expressed as a function of the flaw size. The influence of all other parameters on the POD is considered to be stochastic and normally distributed with a zero mean [5]. This model has been proven in applications where the severity of the flaw for the structural integrity is proportional to the flaw size and the flaw size has the most dominant influence on the POD [6]. An example of such application is the inspection of aircraft turbine engine components, where the size of the crack is the single most important factor that determines its detection [7].

Due to the potential hazards, the nuclear industry is a field where highest safety standards have to be followed. Not only during the electricity production, but also in the process of management and disposal of highly radioactive materials created during the operation. Deep geological disposal, using a system of engineered and natural barriers, is a favoured mean of disposal for high level and long lived radioactive waste [8]. The most important engineered barrier is a canister in which the radioactive material is sealed. In the Swedish and Finnish method of disposal, KBS-3, the canister consists of a load bearing cast iron insert and a copper shell shielding [9, 10]. To make sure that the canister will fulfil its function of long-term safe storage, every component of the canister will be inspected by means of NDT. The role of NDT is to prevent that canisters containing flaws which might jeopardise their function enter the repository. As a main inspection technique, an ultrasonic testing is selected. The reliability model developed in this thesis has been tested on data collected with two ultrasonic inspection methods, one using phased array probes and one using transmitter-receiver longitudinal probes. The reliability of the ultrasonic methods needs to be quantified to confirm the highest level of safety. The NDT reliability study is a part of the risk assessment of the whole repository.

\subsection{Background of the study}

\subsubsection{Engineering materials}

Engineering or structural materials are used in buildings structures, mechanical components and tools. Their primary mission is to carry heavy mechanical loads. They are grouped into three main categories: metals, ceramics and polymers. In addition, there are also composite materials which are a combination of two or three of those. 
Furthermore, some naturally-occurring materials are also considered to be composites for example, wood and bone. When choosing a material for a specific purpose, there are three criteria to be considered [11]. The first is in-service conditions which will dictate the properties required of the material. The second selection consideration is any deterioration of material properties during service life of the structure. Finally, there is the question of economics that also needs to be considered. A trade-off between all three criteria needs to be found when choosing a structural material for the specific purpose.

The properties required of the material are described in terms of the kind and magnitude response to a specific imposed stimulus. All important properties of solid materials may be grouped into six different categories: mechanical, electrical, thermal, magnetic, optical and deteriorative [11]. For each, there is a characteristic type of stimulus capable of provoking different responses. For example, to an applied force, a material response can be described with the modulus of elasticity which is a mechanical property. Another example of a stimulus would be an electric field to which material's response can be described with the electrical conductivity which is an electrical property.

The properties of materials, together with the material structure, processing and performance are components which define the design, production and utilisation of materials. Depending on the processing technique, materials with different microstructure will be created, which will result in different material properties.

\section{Metals}

Metals and metal alloys have the most important role as structural materials. Among the metallic structural materials, steel is the most common. Other important metallic engineering materials are based on light elements such as aluminium, titanium and magnesium. They are frequently used for components of automotive and aerial vehicles.

Two materials chosen for the construction of the canister in a Swedish and Finnish project for long-term storage of radioactive waste are nodular cast iron and copper. Primary selection criteria for the materials were the strength properties for the load bearing insert and sealing properties for the copper shell. 


\section{INTRODUCTION}

Nodular cast iron. Casting is a manufacturing method which allows manufacturing of complicated forms. Nodular cast iron, also known as ductile iron or spheroidal graphite iron is a type of cast iron alloy with carbon and silicon. The amount of carbon is from 2.06 to $4 \%$ and silicon from 1 to $3 \%$. Carbon can be present in cementite or as free graphite. The structure and resulting mechanical properties will depend on the chemical composition, cooling conditions and wall thickness. Mechanical properties of the nodular cast iron are influenced mainly by its metallic matrix. With the addition of magnesium, the growth of graphite in the plane of highest density is obstructed so that sphere-like graphite is formed. The form of the graphite inhibits growth of cracks. Material is good for casting, has a good machinability, poor weldability, high tensile strength with good toughness and good corrosion resistance [12].

The strength properties, good casting properties and good machinability are the main reasons why nodular cast iron was chosen to be the material of the load bearing insert of the canister in the KBS-3 method [13, 14].

Copper. Copper is the oldest metal in use. It is extracted from the copper ore with very small concentration (about $0,6 \%$ ) of copper. It has a high electrical and thermal conductivity which is exceeded only by silver. It is highly chemically and corrosion resistant. It has good cold- and hot-forming properties and good weldability but poor casting properties [12].

High corrosion resistance and radiation protection are the main reasons for selection of copper as a construction material for the shielding shell of the canister [14]. Very well preserved and structurally unchanged copper cannons, found on sunken Viking ships in the Swedish sea demonstrate its longevity.

\section{Material discontinuities}

Materials are built from atoms and molecules that are ideally homogeneously distributed in the volume. Homogeneity of material and uniformity of material properties is desired for most applications. However, absolute homogeneity and continuity never present in any engineering component. Rapid spatial changes of the material homogeneity and continuity inside a component are called discontinuities [15]. The origin of discontinuities and their types depend primarily on the manufacturing process and the 
usage history of the component. Presence of discontinuities is a cause of degradation of properties of ideally homogeneous materials.

\subsubsection{Structural integrity}

Engineering structures need to perform a specified function with high degree of reliability and safety. This is accomplished through good design, manufacturing, test and operational practices. During operational life, structures need to withstand loads imposed on them. These loads can be mechanical (static, dynamic), environmental (temperature, humidity) or chemical.

Loads are the reason for structural degradation and eventually creation of discontinuities in material. Discontinuities may be generated at microscopic level and gradually progress until they become observable (flaws) and eventually compromise the structural integrity (defects).

The cause of most structural failures is either due to the negligence during design, construction or operation of the structure or due to the application of a new design or material, which produces an unexpected (and undesirable) result [16].

One way in which a structure may fail is mechanical failure. This occurs when the structure, or part of it, loses its mechanical integrity to such an extent that it ceases to perform as designed. The mechanical integrity required to function as designed is called the structural integrity. There are many ways in which components can fail mechanically. They may be overloaded, wear out, be exposed to a corrosive environment outside of that for which they were designed. They may also be badly designed, or manufactured, or be operated in an abusive way. However, one of the most frequent causes of failure is the presence of crack-like defects [17].

Very first structures were designed by trial and error. The structures were built and if they failed, the design was improved. With the increased knowledge of materials and operational loads and their interaction, it could have been calculated what kind of loads and of what magnitude, the structure can withstand. Safety margins were imposed with a safety factor, which is dependent on the application and level of uncertainty.

A number of engineering accidents happened, with brittle fractures of normally ductile materials even though the loads were considerably smaller than the material could theoretically withstand. These accidents have shown the need for a better understanding of the mechanisms of fracture. A presence of cracks can modify the local 


\section{INTRODUCTION}

stresses to a such an extent that the elastic stress analysis, based on atomic bonding energies, is not valid any more. Cracks introduce high stress concentrations near their tips and therefore the tensile strength of material is exceeded earlier than when the stress is uniformly distributed in the material.

\subsubsection{Non-destructive testing}

Non-destructive testing (NDT) is an examination performed on an object without affecting its future usefulness. The main reason for the examination is to determine the presence of defects in material, but it can be performed for other purposes as well - e.g. to indirectly measure material properties [18].

NDT plays a significant role in assuring integrity and reliability of engineering structures, minimizing the possibilities of failure [19]. It cannot however guarantee that the failure will not occur. Bad design or improper application may be a cause of the failure even if NDT has been properly applied. The capability of a NDT system to detect small flaws determines the weight of the structure. The smaller the flaw that can be detected with the NDT system, the lighter and thinner the structure can be [15].

There are different NDT methods available for the inspection. Usually they are classified according to the underlying operating physical principle. The common methods are:

- Visual and optical testing (VT)

- Radiographic testing (RT)

- Eddy current testing (ET)

- Ultrasonic testing (UT)

- Liquid penetrant testing (PT)

- Magnetic particle testing (MT)

- Acoustic emission testing (AE)

- Infra-red and thermal testing (IR)

Each of these methods can be further divided into techniques. For example the UT can be used in contact or immersion technique. Choosing the best method and technique for a specific application is a complex task. To properly choose which of 
the methods to apply, an NDT design engineer has to understand and consider the following [20]:

- the physical nature of the material property or imperfection to be inspected

- the underlying physical process that govern NDT methods

- the physical nature of interaction of the probing field with the material

- the potential and limitations of available technology

- economic, environmental, regulatory and other factors

\section{Ultrasonic testing}

The use of sound to determine the integrity of solid objects is probably one of the most widely used NDT methods. The basic principle is fairly simple. Sound waves travel through a solid material and interact with the discontinuities in the object and with the object boundaries. These interactions alter the sound wave. Alterations can be measured and used to detect material discontinuities or geometry of the object or material properties. A tap on the object will set up a vibration at the natural frequency of the object. Any major discontinuity present in the object will change the natural frequency of the object, allowing the change to be heard. The problem with this simple approach is that it requires relatively large discontinuity to change an audible sound. The reason for this is that the wavelength of the audible sound in metals is relatively large and the sound wave will interact only with imperfection of the size comparable with that of the wave [21]. It was not before the discovery of piezoelectric transducers capable of generating ultrasonic waves with small wavelengths, before inspection by means of (ultra)sound entered industrial applications. Some general advantages and disadvantages of ultrasonic testing are provided in table 1.1 .

Two ultrasonic techniques used for the inspection of the Swedish canister parts are described in more details in following sections. These techniques use phased array probes and transmitter receiver longitudinal probes.

Phased array transducers. There are two general designs of the conventional ultrasonic transducers. One is with a single active element that both generates and receives ultrasonic waves, and the other is with two paired elements, one for transmitting and 


\section{INTRODUCTION}

Table 1.1: Characteristics of ultrasonic testing - Advantages and disadvantages of the ultrasonic testing 20 .

\begin{tabular}{l|l} 
Advantages & Disadvantages \\
\hline High penetration depth & Significant operator training \\
High sensitivity & Often requires contact using couplant \\
High accuracy & Cannot detect planar flaws perpendicular to the \\
& wavefront \\
Rapid testing & Intrinsically a point-by-point measurement \\
Can test complex geometries & Can be expensive \\
Can measure material properties & \\
Can test on all materials & \\
Portable & \\
Safe &
\end{tabular}

one for receiving. Phased array probes commonly consist of a transducer assembly with from 16 to as many as 256 small individual elements that can each be triggered separately [22]. These may be arranged in a strip (linear array), a ring (annular array) or a more complex shape. The same as the conventional transducers, phased array probes may be designed for direct contact use, as part of an angle beam assembly with a wedge, or for immersion use with sound coupling through a water path. The frequencies of the transducers are most commonly in the range from $2 \mathrm{MHz}$ to $10 \mathrm{MHz}$. A phased array system consists of several advanced modules. A module for triggering the multi-element probe, a module for receiving and digitizing the returning echoes, and a module for plotting echo information in various formats. Superior to the conventional transducers, phased array systems can sweep a sound beam through a range of refracted angles or along a linear path, and dynamically focus at a number of different depths. This increases both flexibility and capability of the inspection system [23].

Transmitter-receiver longitudinal transducers. In many applications, one transducer is used for both transmission and reception of the ultrasonic waves in so called pulse-echo mode [24]. One major disadvantage of this method is that the reflection from the front surface of the object will obscure any signal in the zone close to the surface. This zone is also called the "dead zone". When searching for flaws in this region, it is an advantage to use two transducers instead of one, one as a transmitter and one 


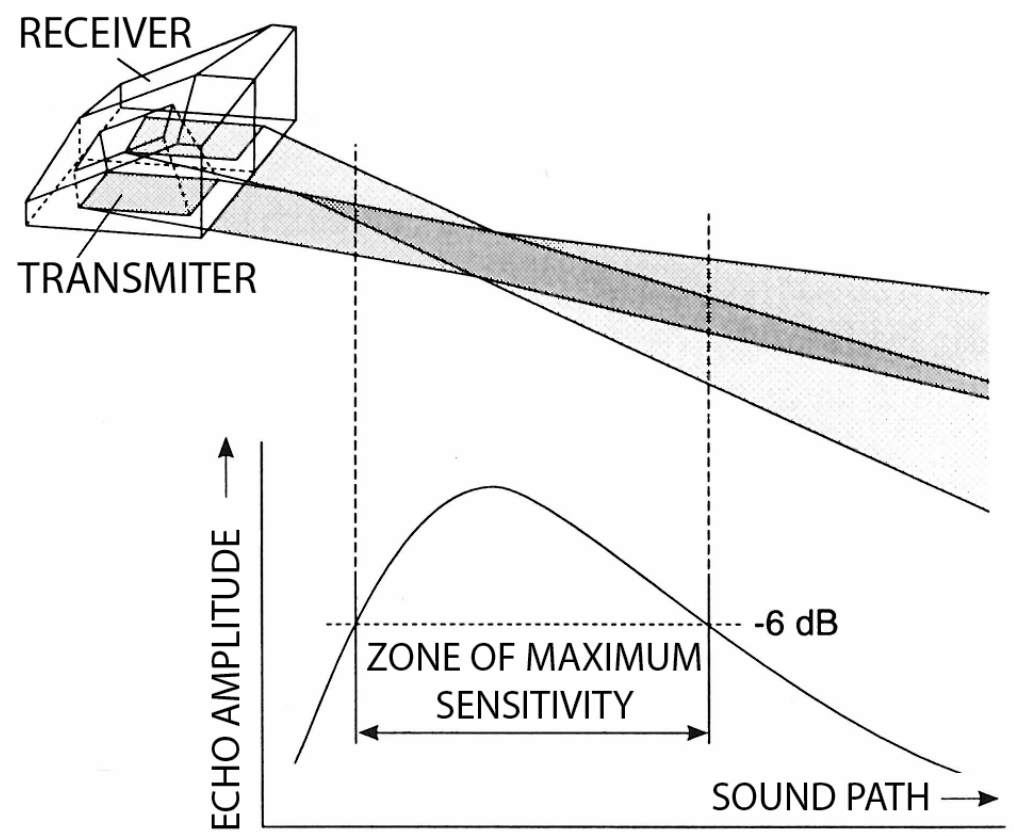

Figure 1.1: Angled dual transducer - Schematic representation of the angled dual transducer

as a receiver, coupled in one casing with the buffer [25, 26]. A schematic view of the angled dual transducer is shown in figure 1.1. By choosing the roof angle of transducers, the zone of maximum sensitivity and the dead zone can be altered [27]. Transmitterreceiver longitudinal (TRL) probes are ultrasonic twin crystal probes which generate angled longitudinal waves within the component. The separate transmitting and receiving crystals are toed-in towards each other providing pseudo focusing. The depth of focusing is determined by the angle of toe-in of the crystals. Maximum sensitivity and the minimum beam width typically occur in front of the geometrical crossing region of the beams from each crystal.

Performance of conventional ultrasonic inspection techniques for cast iron is degraded because of the coarse grain structure. The coarse structure causes attenuation losses and scattering noise and as a result to low signal-to-noise ratio (SNR). To overcome these problems, low frequency TRL ultrasonic probes are suggested for the inspection to reduce backscattered noise and therefore enhance the SNR, especially in close to surface regions [28. The TRL probes are used for the inspection of the close to surface region of the cast iron insert of the Swedish canister. 


\section{INTRODUCTION}

Change of an ultrasonic wave amplitude as the wave propagates through the material

The amplitude of the ultrasonic wave changes as the wave propagates through the material. The most abrupt changes happen on the boundaries between materials with different acoustic impedance properties [25]. Additional change of the amplitude is caused by the diffraction of the non-planar wave [29]. Finally, the amplitude will attenuate as the wave propagates because of the different absorption mechanisms and scattering on the material inhomogeneities, known as the ultrasonic attenuation [29].

Change of amplitude on material boundaries. When the longitudinal plane wave impinges normal to a flat surface, a part of the wave will be reflected and a part of it will be transmitted through the boundary. The amplitudes of the reflected and transmitted waves can be found from the condition that the displacements and stresses must be continuous on the boundary [25]:

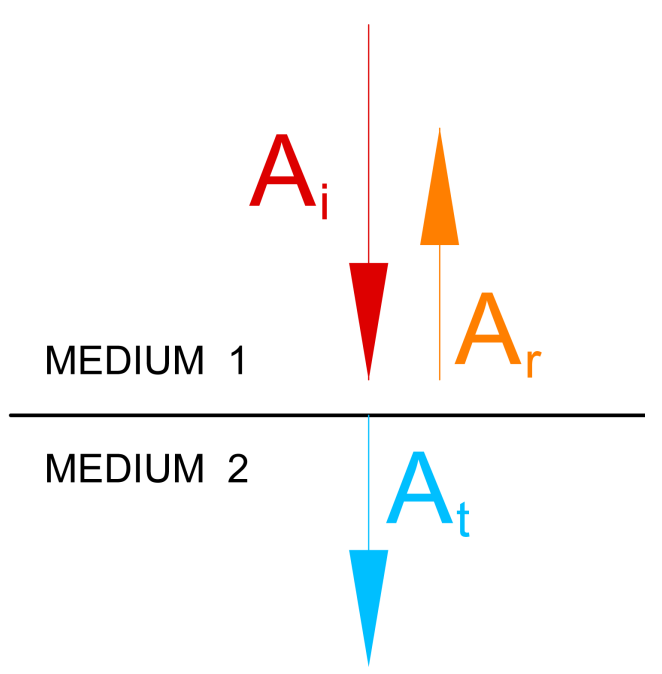

Figure 1.2: Ultrasonic wave amplitude on the material boundary - Reflection and transmission of the wave on the boundary between two materials with different acoustic impedance properties

$$
R=\frac{A_{r}}{A_{i}}=\frac{Z_{2}-Z_{1}}{Z_{2}+Z_{1}}
$$

and 


$$
T=\frac{A_{t}}{A_{i}}=\frac{2 Z_{2}}{Z_{2}+Z_{1}}
$$

where $R$ and $T$ are the reflection and transmission coefficient respectively and $A_{i}, A_{r}$ and $A_{t}$ the amplitude of the incidence, reflected and transmitted wave respectively, as illustrated in figure 1.2. $Z_{1}$ and $Z_{2}$ are the acoustic impedances of the first and second material. For example, if a longitudinal wave from water $\left(Z_{1}=1.48 \times 10^{6} \mathrm{~kg} / \mathrm{m}^{2} \mathrm{~s}\right)$ impinges on the boundary with copper $\left(Z_{2}=41.6 \times 10^{6} \mathrm{~kg} / \mathrm{m}^{2} \mathrm{~s}\right)$, equations 1.1 and 1.2 will give $R=0.93$ and $T=1.93$. The amplitude of the reflected wave will be $93 \%$ of the amplitude of the incidence wave and the amplitude of the transmitted wave will be $193 \%$ of the amplitude of the incidence wave. The higher amplitude of the transmitted wave seems like a paradox. The intensity of the wave is calculated from the product of the wave pressure and the sound impedance which in water is much smaller than in copper. If the wave that propagates in copper impinges on the boundary with water, the amplitude of the reflected wave back in copper will be $93 \%$ of the incidence wave amplitude ( $R=-0.93$ from equation 1.1 , the minus sign indicates a phase change). The amplitude of the transmitted wave into water amounts only about $7 \%$ of the amplitude of the impinging wave $(T=0.07$ from equation 1.2$)$.

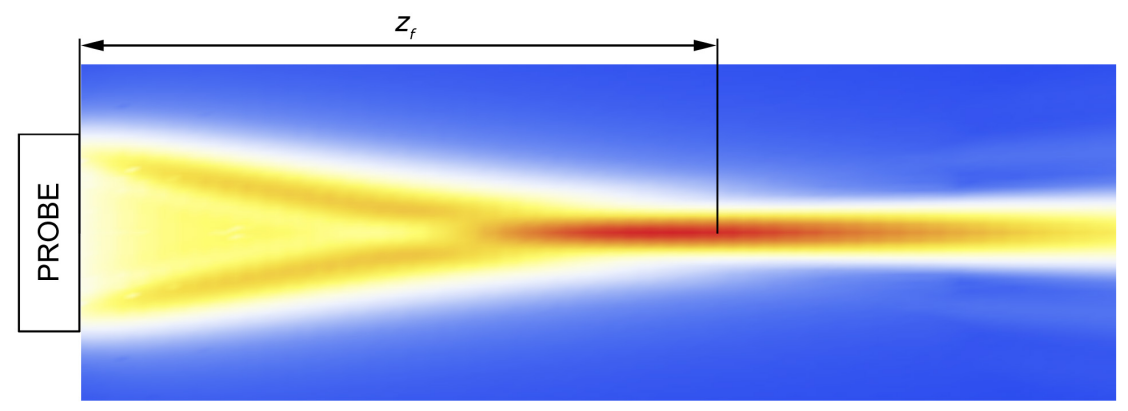

Figure 1.3: Ultrasonic focused beam - Computer simulation of the focused ultrasonic sound beam generated by the phased array probe, focused at depth $z_{f}$

Sound beam spreading The amplitude of the plane wave, propagating through the ideal material without attenuation, does not change with the distance. The wave generated with the real, finite-size transducer, will spread out into a complex and position dependant field pattern [30. This effect is known as a beam spreading. Furthermore, 


\section{INTRODUCTION}

in phased array ultrasonics, by using different excitation time of individual elements, the sound field can be formed by varying the interference conditions, i.e. the focused sound beam can be generated [23]. An example of such a focused beam is shown in figure 1.3. The spreading of the sound beam can be estimated experimentally [31] or by simulation. The change of the amplitude due to the sound beam spreading of the single frequency wave on the acoustic axes of the phased array transducer with the sound beam focusing can be described by a function in the form:

$$
A_{d}(z)=P(z) \exp \left(C z^{2}\right)
$$

where $A_{d}$ is a change of the amplitude with the distance $z$ because of the beam spreading, $P$ is a polynomial in $z$ and $C$ is a coefficient of the exponential term.

Attenuation of the sound wave The attenuation of the plane sound wave caused by the absorption and scattering in real materials, can be approximated by the exponential function [25]:

$$
A_{m}(\alpha, z)=A_{0} \exp (-\alpha z)
$$

where $A_{m}$ is the reduced amplitude after the wave has travelled a distance $z$ from the initial location, $A_{0}$ is the amplitude at the initial location and $\alpha$ is the material attenuation coefficient.

Overall Change of Amplitude The overall change of the amplitude $A_{o}$ of the sound wave propagating through the material is a product of the change of the amplitude due to the sound beam spreading and ultrasonic attenuation:

$$
A_{o}(\alpha, z)=A_{d}(z) A_{m}(\alpha, z)
$$

This is illustrated in the diagram in figure 1.4 . The dashed curve shows a change of amplitude with the depth of the focused ultrasonic beam, as a result of the beam spreading. The maximum amplitude $A_{\max }$ is achieved in the focal depth $z_{f}$. The dotdashed line shows the exponential decrease of the amplitude with the depth due to the ultrasonic attenuation. The solid curve represents the resulting, overall amplitude curve. It is important to notice that the maximum amplitude of the resulting curve 
$A_{\text {max }}^{\prime}$ is lower than it would be if the wave would propagate without attenuation. Also, the maximum amplitude is achieved in shallower depths $z_{f}^{\prime}$; the maximum is shifted towards the probe.

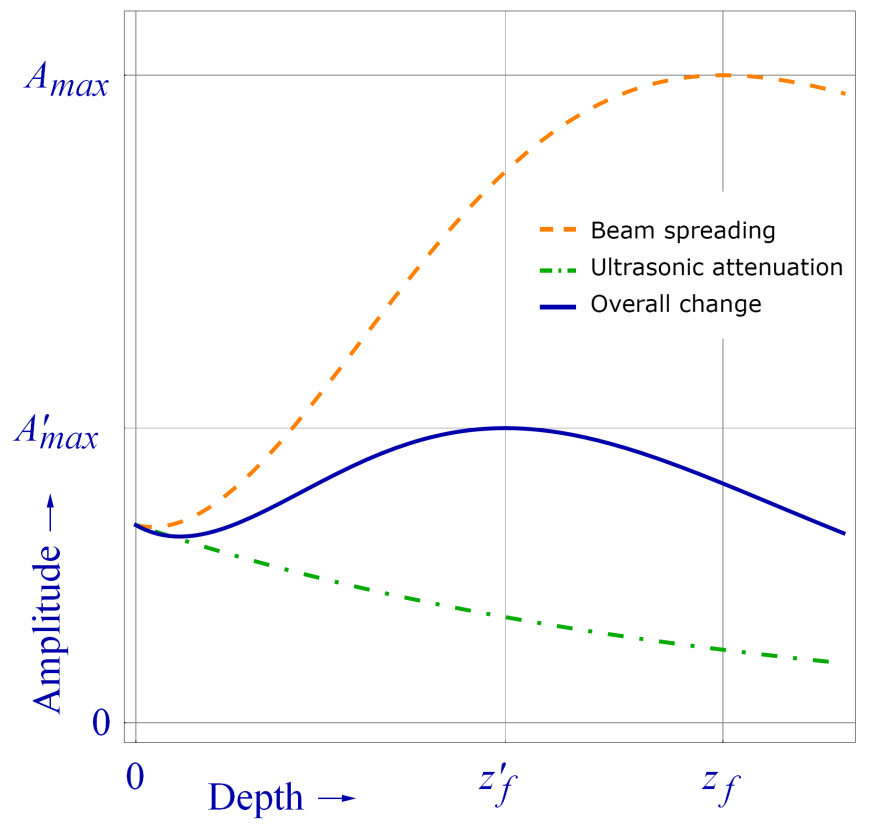

Figure 1.4: Overall change of amplitude - Overall change of amplitude as the sound wave propagates through the material

\section{From a discontinuity to a defect}

It is very important to distinguish between some important NDT terms, which are often mistakenly used interchangeably. In this thesis these terms are used as defined in ASTM E1316-07b Standard Terminology for Nondestructive Examinations [2]. As already mentioned, absolute homogeneity and continuity never exists in any engineering component. Spatially sharp departures from material homogeneity and continuity are called a discontinuity. A discontinuity that may be detected by the NDT system is called a flaw. A flaw will cause that the NDT system produces an indication. This indication needs to be evaluated to determine if the flaw meets specified acceptance criteria. If it does not, the flaw is characterized as a defect and the component can not be used further without corrective actions. 


\section{INTRODUCTION}

\subsubsection{Nuclear waste management}

Nuclear power plants provide about $11 \%$ of the world's electricity [32]. According to the Nuclear Energy Institute (NEI) as of September 2011, 30 countries worldwide are operating 433 nuclear reactors for electricity generation and 65 new nuclear plants are under construction in 14 countries [33]. Table 1.2 shows countries generating the largest percentage of their electricity from nuclear power plants. Even if it is still considered by many as clean and safe energy [34], in light of the accident in Fukushima and the accident in Chernobyl, many countries are rethinking their nuclear energy policies. Regardless of whether the nuclear power continues to be used for electricity generation or not, the greatest problem of the nuclear era - the nuclear waste problem - still needs to be solved. Even if all nuclear plants are closed today, there is still a large amount of nuclear waste that needs to be disposed of in a safe manner. The responsibility for the disposal lies on those who enjoyed the benefits of the nuclear power and must not be left for the next generations to solve [35].

Table 1.2: Electricity production from nuclear energy - Countries generating the largest percentage of their electricity from nuclear energy in 2010 [3]

\begin{tabular}{c|c} 
Country & $\begin{array}{c}\text { Percent of the total electricity } \\
\text { generated from nuclear energy }\end{array}$ \\
\hline France & 74.1 \\
Slovakia & 51.8 \\
Belgium & 51.1 \\
Ukraine & 48.1 \\
Hungary & 42.0 \\
Armenia & 39.4 \\
Sweden & 38.1 \\
Switzerland & 38.0 \\
Slovenia & 37.4 \\
Czech Rep. & 33.2 \\
Bulgaria & 33.0 \\
Korea Rep. & 32.2 \\
Japan & 29.2 \\
Finland & 27.5 \\
Germany & 27.3
\end{tabular}


Nuclear waste is defined as liquid, solid, or semi-solid waste products possessing at least some amount of radioactive elements. It can be categorized by the radioactive content. Low-level waste (LLW) contains radionuclides with low activities and short half lives and generates no heat. Intermediate-level waste (ILW) may contain radionuclides with low to intermediate activities and short to long half lives, generating no to negligible heat. High-level waste (HLW) contains radionuclides with high activities, long or short half lives or both, and generates heat [36]. By far the largest volume is LLW, a smaller volume of waste is ILW and a very small volume of waste is HLW. Although of small volume, HLW contains the major part of the radioactivity produced in the nuclear fuel cycle [37.

The most waste management specialists agree that the geological disposal, using a system of engineered and natural barriers, is the preferred mean of disposal of high level and long lived radioactive waste [8]. Justification is based on natural analogues e.g. with an uranium mine in Oklo in Gabon where the evidence of nuclear reactors which were active 2000 million years ago were found. Radionuclides, also present in HLW, remained contained over geological periods and many decayed totally within the ore body [38]. Deep burial of HLW in geological formations relies on a multiple-barrier concept. A number of engineered and natural barriers are combined to isolate wastes for sufficient time to permit radioactive decay to occur to appropriate levels [39]. The aim of the underground repository is to ensure that the potential impact of disposal on the environment and on the population's safety is within the requested limits, for a long-term period after closure. There is a series of uncertainties connected with a deep geological disposal. Among the most important causes of them are [40]:

- considerations for safety over very long time periods, typically up to thousands or even millions of years,

- large spatial areas taken into account,

- absence of exact mathematical models for the very complex repository system, and the subsequent uncertainty introduced by any conceptual and/or computer models used instead,

- lack of accuracy in the data values, being a result of either the imprecision in values or the use of implicit measurement methods,

- subjective judgement by experts regarding the validity of the models. 


\section{INTRODUCTION}

The Swedish method of the deep geological disposal is presented in the next section.

\section{KBS-3 method for deep geological disposal}

According to the Swedish law the reactor operator has to demonstrate that the spent nuclear fuel can be handled and disposed in a safe manner before the permission for operation of a nuclear power plant can be granted. After years of research, Swedish Nuclear Fuel and Waste Management Company (SKB) came up with a solution according to which the spent nuclear fuel should be encapsulated in copper and embedded in bentonite clay at a depth of 500 metres in the crystalline bedrock. The means of achieving this are elaborated in the set of reports under the name KBS-3 method [9, 41, 42, 43, 44,. KBS-3 describes how a system for safe final disposal of spent nuclear fuel can be designed based on the state-of-the-art knowledge.

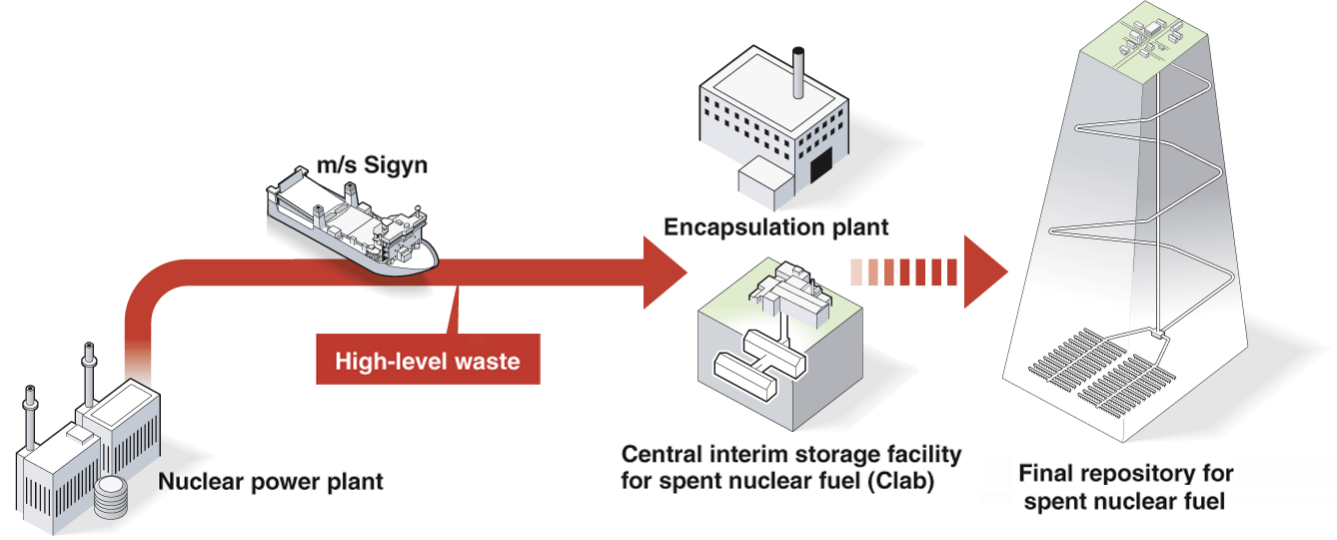

Figure 1.5: KBS-3 method - Swedish system for management and disposal of high-level radioactive waste (Illustration courtesy of SKB)

Principal components of the system for management and disposal of HLW are shown in figure 1.5. When the fuel is taken out of the reactor, it is stored in water-filled pools at the nuclear power plant for at least nine months. During this time its radioactivity declines considerably - by about 90 percent. The spent fuel is then transported to the central interim storage facility for spent nuclear fuel. Since all Swedish nuclear reactors are located on the coast, the system is based on sea transport with a specially built ship. The fuel is kept in interim storage for about 30 years during which the 
fuel's radioactivity declines by an additional 90 percent. Once the final repository is put into operation, the spent fuel will be transported from the interim storage to the encapsulation plant, where it will be encapsulated in tight, corrosion resistant and load bearing canisters. The canisters will be deposited in crystalline rock at a depth of 500 metres. Since one of the basic guiding principle is a very high level of safety, the multiple barrier principle is applied. The canisters will be surrounded by a buffer which prevents the flow of water and protects them and the cavities in the rock. After all canisters have been deposited, the final repository will be sealed by filling the tunnels and shafts with a mixture of sand and bentonite clay. The radioactive substances in the spent fuel shall be contained in the final repository until the level of the radioactivity drops to a level of the radioactivity of uranium ore which is found in nature. This is estimated to be about 1000000 years. The ways of achieving protection are based on containment of radioactive substances and dispersal at a slow rate with high dilution.

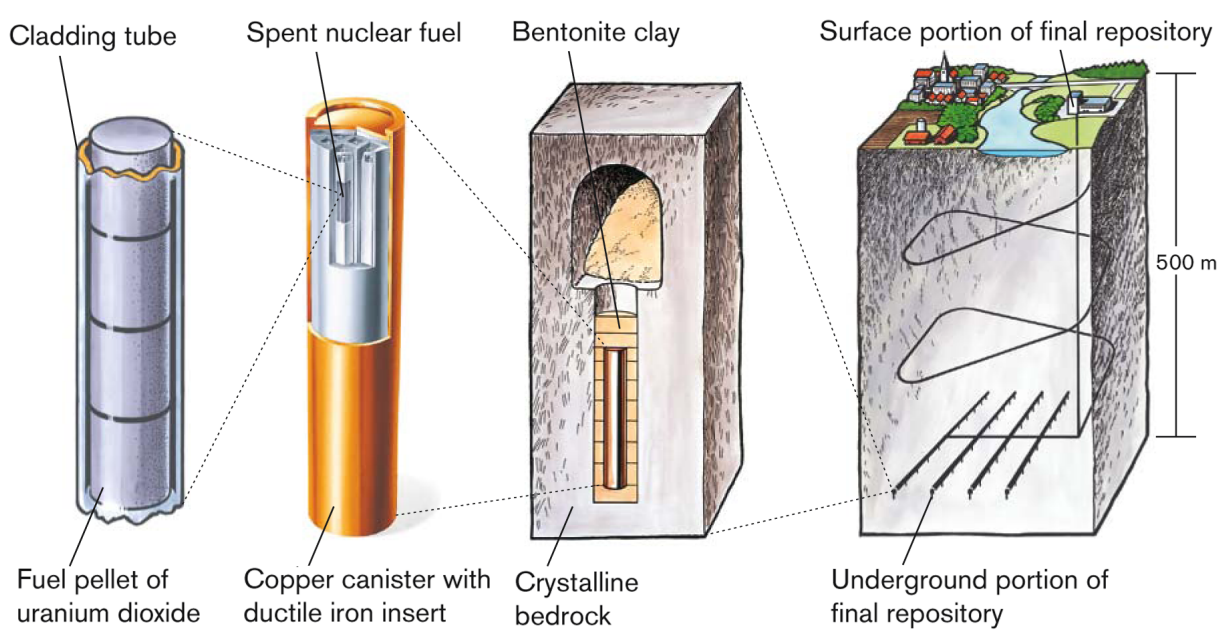

Figure 1.6: Barriers in the final repository - The role of the barriers is to prevent contact of the spent nuclear fuel with the environment (Illustration courtesy of SKB)

The barriers that prevent contact of the spent nuclear fuel with the environment are shown in figure 1.6. The function of the canister is to contain the spent nuclear fuel and prevent the release of radionuclides into the surroundings. It also shields radiation and prevents criticality. The buffer is a clay containing swelling material. The buffer surrounds the canister and fills the space between the canister and the 


\section{INTRODUCTION}

bedrock. The buffer shall prevent flow of water and protect the canister. In case the containment provided by the canister should be breached the buffer shall prevent and retard the dispersion of radioactive substances from the canister to the bedrock [45, 46, 47. 48. The primary function of the bedrock is to protect the canister and the buffer. Additionally, it also retards the transport of the radionuclides [49]. The spent nuclear fuel, an uranium oxide, can be considered as a barrier itself because due to its strong ceramic bonds, it has a very low solubility in water [50].

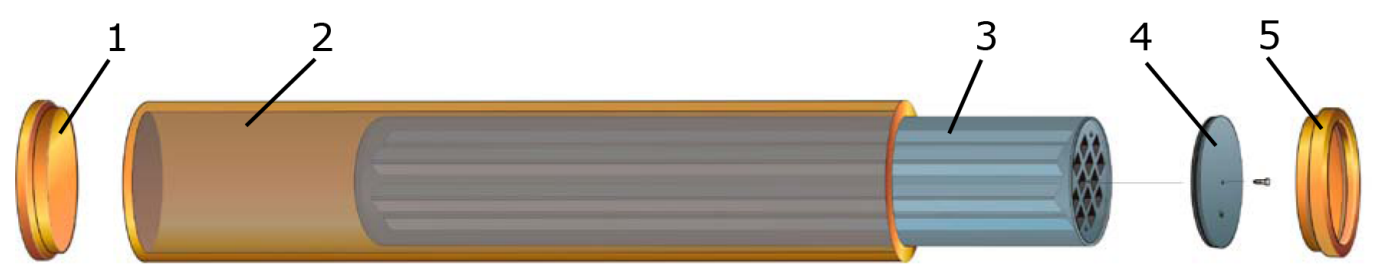

Figure 1.7: Exploded view of the canister - Canister components: 1 - copper base, 2 - copper tube, 3 - cast iron insert, 4 - steel lid, 5 - copper lid (Illustration courtesy of SKB)

Canister. The canister is the most important engineered barrier isolating the spent nuclear fuel from the surroundings. It is a container with a tight, corrosion resistant shell of copper and a load bearing insert in which the spent nuclear fuel is placed. The components of the canister, a cast iron insert with steel tube cassette, steel lid, copper tube, copper lid and copper base, are illustrated in figure 1.7. Since the canister shall contain the various types of spent nuclear fuel from the Swedish nuclear power programme, there are two versions of insert. One is adapted to the dimensions of the boiling water reactors (BWR) assemblies and one adapted to the pressurized water reactor (PWR) assemblies. The insert contains channels for the fuel assemblies, 12 in the BWR version and 4 in the PWR version, as illustrated in figure 1.8. Once the spent fuel is loaded into the channels, the lid is welded to the tube with friction stir welding (FSW) technique. The maximum total weight of the canister, including fuel, is $24700 \mathrm{~kg}$ for BWR and $26800 \mathrm{~kg}$ for PWR.

There are several design requirements related to the barrier function in the KBS-3 repository that shall be verified for the reference canister. The canister shall withstand an isostatic load of $45 \mathrm{MPa}$, being the sum of maximum swelling pressure and maximum groundwater pressure. The corrosion barrier of the canister shall remain intact 

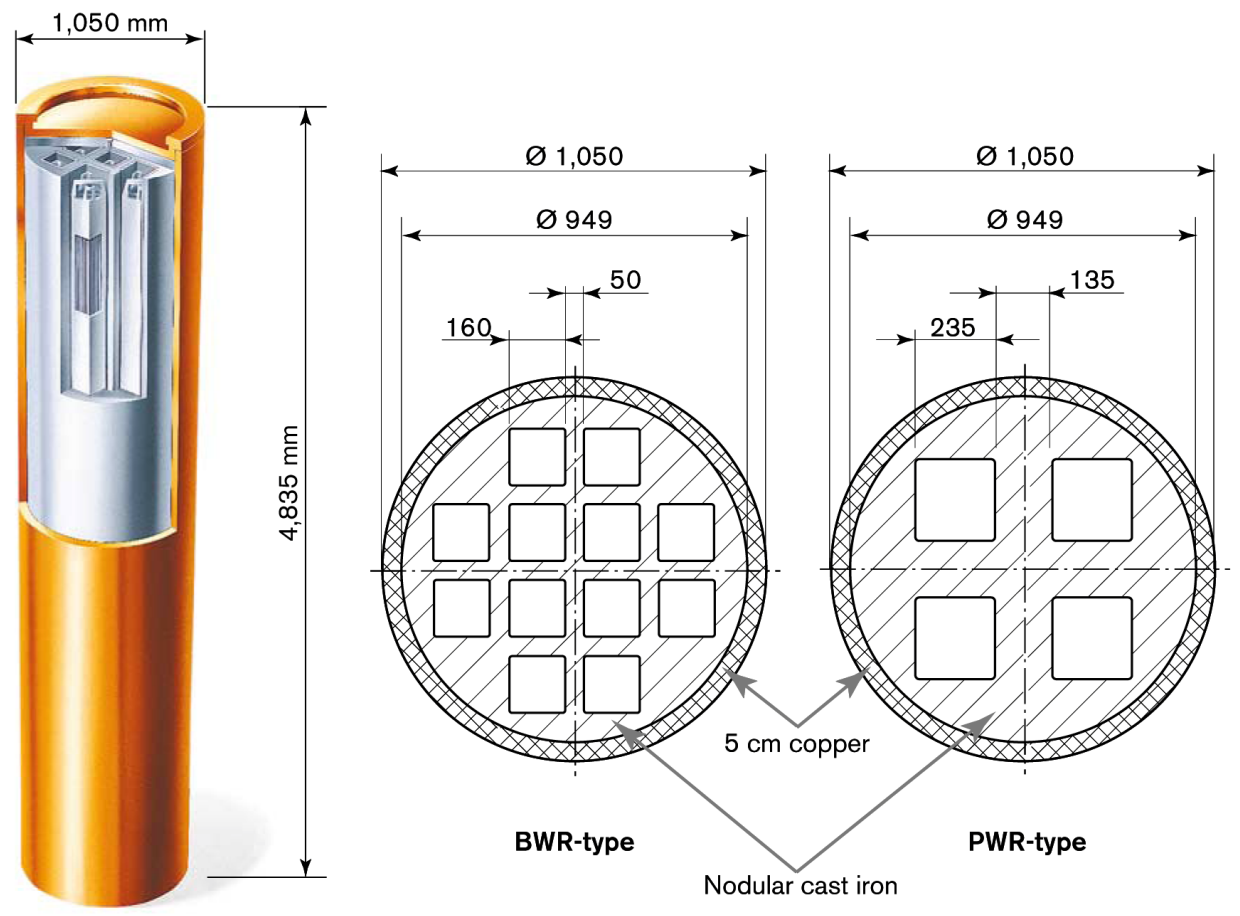

Figure 1.8: Different versions of the insert - Principle dimensions of the canister and cross section showing the difference between two inserts. BWR-type with 12 fuel channels and PWR-type with four fuel channels (Illustration courtesy of SKB) 


\section{INTRODUCTION}

after $5 \mathrm{~cm}$ shear movement at a velocity of $1 \mathrm{~m} / \mathrm{s}$ for buffer material properties of a $2050 \mathrm{~kg} / \mathrm{m}^{3}$ Ca-bentonite, for all locations and angles of the shearing fracture in a deposition hole, and for temperatures down to $0{ }^{\circ} \mathrm{C}$. The insert should maintain its pressure-bearing properties to isostatic loads. The copper shell shall have a nominal thickness of $5 \mathrm{~cm}$ to withstand the corrosion load. The spent fuel properties and geometrical arrangement in the canister are such that criticality shall be avoided even if water should enter the canister. With respect to the design requirements for the spent nuclear fuel, it shall be verified that the dimension of the insert channel tubes are sufficient to accommodate the spent fuel to be deposited and that the canister is marked with a unique identity. With respect to manufacturing and operation requirements, it shall be verified that the canister can be lifted safely by its lid even when it exerts the highest stresses during an unplanned stoppage in canister lowering.

Two large-scale pressure tests have shown that the canister remained intact under the iso-static load of $130 \mathrm{MPa}$, which is a large safety margin [51]. The experiments showed a good agreement with the finite elements predictions [52]. The post-test analysis of the defects showed that the cracks are most probably initiated at the large subsurface inclusions in the zone of the tensile stresses and grow by stable tearing. When the canister eventually fails, plastic collapse is more likely than fracture from casting defects.

Manufacturing flaws which might occur in canister components. A number of manufacturing methods are being considered for production of canister parts including two different types of welding and it is necessary to understand the effects which material, manufacturing and welding flaws will have on the durability of the product in its service environment. Manufacturing methods are the casting of the nodular iron, the casting of the copper ingot, the extrusion of the copper tube, the forging of the copper lid and base and the friction stir welding (FSW) of the copper lid and base to the tube.

Studies have been conducted to identify the flaws which might occur in canisters produced serially, to classify the possible flaws that have been identified according to their likely effect on durability of the canister in manufacturing and in the service environment, and to identify flaw characteristics essential for the non-destructive testing [53, 54, 55, 56]. The origin and types of discontinuities depend primarily on 
Table 1.3: Flaws which might occur in direct casting process of the cast iron insert 53 .

\begin{tabular}{l|l|l} 
Defect type & Comment & Method of detection \\
\hline Dross inclusions & Should float out & Ultrasonic \\
\hline Cold shuts & Should be prevented by rapid pour & Ultrasonic \\
\hline Gas porosity & Should float out & Ultrasonic \\
\hline Shrinkage cav- & $\begin{array}{l}\text { Should be very limited owing to con- } \\
\text { ity }\end{array}$ & Ultrasonic \\
\hline Segregation & $\begin{array}{l}\text { Graphite may segregate to top of } \\
\text { casting during long solidification pe- } \\
\text { riod }\end{array}$ & $\begin{array}{l}\text { Micro-structural examina- } \\
\text { tion / mechanical test }\end{array}$ \\
\hline Bad structure & $\begin{array}{l}\text { Graphite may coarsen during long so- } \\
\text { lidification and cooling periods }\end{array}$ & $\begin{array}{l}\text { Micro-structural examina- } \\
\text { tion / mechanical test }\end{array}$ \\
\hline Shrinkage & $\begin{array}{l}\text { Could occur in webs as a result of } \\
\text { rapid cooling. Choice of nodular iron } \\
\text { cracks }\end{array}$ & $\begin{array}{l}\text { No satisfactory test proce- } \\
\text { dure } \\
\text { minimize any problem }\end{array}$ \\
\hline Inclusions & $\begin{array}{l}\text { Particles from mould may detach } \\
\text { during pour and be included in the } \\
\text { casting }\end{array}$ & Ultrasonic
\end{tabular}

the manufacturing processes and the service histories of engineering components. In some cases, the operational environment may induce the growth and development of pre-existing discontinuities. Discontinuities in structures may originate at any manufacturing step and may be introduced during the component use, maintenance, and repair. An understanding of the origin of discontinuities is useful in determining the type and features of flaws (i.e. discontinuities that may be detected with NDT) that may be expected in a component. Awareness of the characteristics, locations, and orientations of flaws is most helpful and sometimes critical in their detection and evaluation. Flaws that might occur in the process of direct casting of the cast iron insert are given in table 1.3. Flaws that might occur in the copper tube during extrusion process are given in table 1.4. Studies concluded that flaw parameters that are essential to the quality of ultrasonic testing are flaw position in the object (depth), orientation, size, surface roughness, closure and tip radius. It is important to notice that there are several parameters identified as essential for the flaw detection, not only its sheer size. 


\section{INTRODUCTION}

Table 1.4: Flaws which might occur in the extrusion process of the copper tube [53]

\begin{tabular}{|c|c|c|}
\hline Defect type & Comment & Method of detection \\
\hline Inclusions & $\begin{array}{l}\text { May arise as clouds or stringers of } \\
\text { oxide or foreign material such as re- } \\
\text { fractory. May disrupt Electron Beam } \\
\text { Welding. Effect on corrosion perfor- } \\
\text { mance unknown }\end{array}$ & Ultrasonic \\
\hline Coarse grains & $\begin{array}{l}\text { Arise during piercing, should be re- } \\
\text { moved by machining. May be due } \\
\text { to friction at the die or hot short- } \\
\text { ness. May also arise during drawing } \\
\text { to increase diameter and reduce wall } \\
\text { thickness }\end{array}$ & Ultrasonic \\
\hline Hot tearing & $\begin{array}{l}\text { Cracks arising from friction at the ex- } \\
\text { trusion die and/or segregation of im- } \\
\text { purities at the grain boundaries in } \\
\text { the ingot }\end{array}$ & $\begin{array}{l}\text { Visual inspection as ex- } \\
\text { truded, die penetrant after } \\
\text { machining }\end{array}$ \\
\hline Speed cracking & $\begin{array}{l}\text { Circumferential surface cracks. Con- } \\
\text { trolled by adjustment of extrusion } \\
\text { temperature and extrusion rate }\end{array}$ & $\begin{array}{l}\text { Visual as extruded, die pen- } \\
\text { etrant after machining }\end{array}$ \\
\hline Bad shape & $\begin{array}{l}\text { Could lead to failure to achieve the } \\
\text { specified dimensions after machining }\end{array}$ & $\begin{array}{l}\text { Measurement before ma- } \\
\text { chining, visual inspection } \\
\text { after machining }\end{array}$ \\
\hline
\end{tabular}

NDT of canister components. To guarantee that the canister will fulfil its function, different kinds of tests are planned at different stages of canister manufacturing. NDT of the canister components aims to find flaws in the material, to characterize them and to determine if they satisfy acceptance criteria. If the flaws that do not satisfy acceptance criteria (i.e. defects) are found in the material, the component can not be used for the storage of the radioactive material and is therefore rejected. The requirement is to inspect $100 \%$ of material of every canister. As a primary inspection method ultrasonic testing is chosen. Several inspection techniques will be applied to fulfil the requirement. For the purpose of validation of the reliability model developed in this thesis, inspections techniques with the phased array probes and transmitter-receiver longitudinal probes are investigated. 


\subsection{Organisation of the thesis}

The thesis is organized as follows: in Chapter 2, the state of the art in reliability of NDT is presented. First, a general definition of reliability and one that defines the reliability of NDT, are given. They are followed by the modular model concept that breaks the NDT system into constituting modules. The principles of signal detections theory are explained afterwards, followed by the definition of receiver operating characteristics. The chapter is concluded with the introduction of the POD curve and the role of the computer simulation in NDT.

Chapter 3 shows limitations of the conventional, signal response reliability model when several parameters are important both for the severity of the flaw for the structural integrity and for the POD of the flaw. The problem was encountered when evaluating inspection data of the ultrasonic inspection techniques used to inspect the canister for nuclear waste storage. Also, the problem of evaluating large amount of inspection data, especially if the part is inspected with more than one sensor, is presented.

Chapter 4 presents a novel multi-parameter POD model which enables the POD to be calculated as a function of several influencing parameters. The application of the model is first demonstrated on the ultrasonic inspection data of the cast iron component, where the depth extension and width of the flaw are parameters that influence flaw severity for structural integrity and as such are taken in the multi-parameter model calculation. The advantage of the multi-parameter model over the conventional one is given at the end by directly comparing the results of the two models. In the second experiment, the results of the application of the multi-parameter model on the ultrasonic inspection of thick copper components with high and variable material attenuation are presented. The POD is calculated as a function of the flaw depth, angle and the ultrasonic attenuation coefficient.

Chapter 5 presents a way how to spatially present the POD data when the depth of the flaw is taken as an influencing parameter in the multi-parameter POD model. This representation of the data, overlaid on the component geometry is called a volume POD. Also, a way to perform a POD data fusion when a component is inspected with more than one inspection. Finally, a way how to use the overall volume POD diagram to optimize the NDT system is shown. 
In the concluding Chapter 6 the most important contributions are summarized, the limitations of the model are given, as well as pointers for the continuation of the research. 


\section{State-of-the-Art in Reliability of Non-destructive Testing Systems}

The flaws exist in every engineering structure. This is recognized and it is tolerated as long as they do not compromise the structural integrity. To meet this condition, components need to be inspected and flaws timely detected. NDT is principally used to detect flaws in a material, without affecting future usefulness of the inspected component. In order to determine if the NDT system is capable to detect flaws that could compromise the structural integrity, its capability to detect flaws needs to be quantified. The minimum size of the flaw that can be detected by the system needs to be established. This is done in the experiment, by inspecting the specimens containing flaws of known size. By trying to detect smaller and smaller flaws, one would hope to determine the minimum size of the flaw that can be detected with the evaluated system. However, the experience is showing that repeated inspections of the same flaw do not result in consistent detections. The same flaw might be detected or missed by the NDT system, depending on many parameters. The reason for this is that an inspection is subject to the inevitable uncertainty that attends all measurements [57]. This uncertainty is a key element of NDT [58] and that is why the capability of the NDT system to detect flaws is described in terms of reliability [59].

First NDT reliability studies arose at the end of the 1960s in the aerospace industry. Pretty soon, the studies spread into all other fields where a missed defect can cause fatalities, environmental damage or big financial losses. A series of European-American Workshops on NDT reliability resulted in the identification of the building blocks of an 


\section{STATE-OF-THE-ART IN RELIABILITY OF NON-DESTRUCTIVE TESTING SYSTEMS}

NDT system, described by the modular model. A way to present an NDT system's capability to detect flaws is by plotting the detection rate against the false alarm rate in a receiver operating characteristic (ROC) diagram. A probability of detection (POD) curve is considered to be a standard method to quantify the NDT capability of detecting flaws. There is also a substantial work to incorporate a computer simulation of the inspection process to improve the understanding of the phenomena and simplify the process of the POD estimation.

\subsection{Definition of reliability}

There are many different views and interpretations of reliability [60], but most will agree that a reliable product is of better quality than the one being less reliable. Reliability can be seen as a measure of performance and there is a need to quantify it. Engineering definitions of reliability like "the probability that a system, vehicle, machine, device and so on will perform its intended function under operating conditions, for a specified period of time" 61] or "characteristic of an item expressed by the probability that it will perform its required function in the desired manner under all the relevant conditions and on the occasions or during the time intervals when it is required so to perform" 62] need to be revised before transferred on NDT systems.

When defining a reliable measurement equipment a more appropriate definition is "the extent to which an experiment, test, or measuring procedure yields the same results on repeated trials" [63]. A reliable NDT system is defined as "one that, when rigorously applied by a number of teams, consistently detects all defects of concern" [64]. The most comprehensive definition of NDT reliability in the sense understood in this thesis is "the degree that a NDT system is capable of achieving its purpose regarding detection, characterization and false calls" where "the NDT system consists of the procedures, equipment and personnel that are used in performing the inspection" 3]

\subsection{Modular model}

A need to reconcile views between different industries on how to determine reliability of NDT, resulted in a series of European-American Workshops on NDT reliability. One 
concept that emerged from these workshops is a modular model. To ease the assessment of the reliability of a NDT system, its overall reliability can be expressed as a function of three constituting modules [65]. These modules are the intrinsic capability (IC), application parameters (AP) and human factors (HF). Intrinsic capability defines the technical capability of the system, generally considered as an upper bound of performance. Application parameters are an application specific set of parameters that will influence the inspection, when compared for example to the one performed in ideal, lab conditions. The human factors describe all those parameters that will be brought into the system because of the human involvement in the process. Before all, that is the influence of the inspector that performs the inspection. The human factor influence is hard to define and there are very different opinions about the importance and magnitude of it. One can read very different opinions about the significance of the human factors. They range from "the human conducting the inspection is the most significant variable in the process" [5] to "whilst human factors are important variables in NDT procedures, they are often found not to be as important as other operational and physical variables" 66

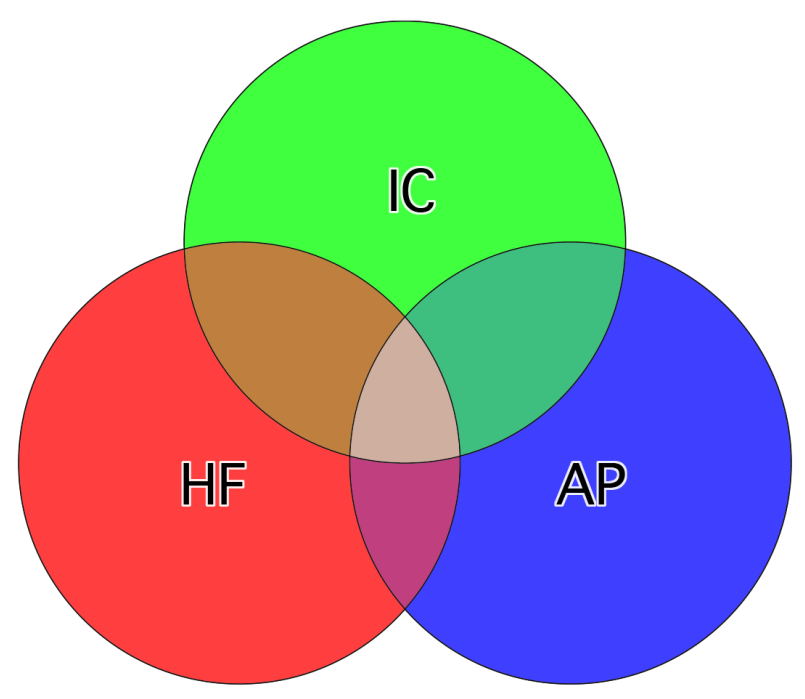

Figure 2.1: Modular model - Schematic representation of the constituting modules of the NDT system 


\section{STATE-OF-THE-ART IN RELIABILITY OF NON-DESTRUCTIVE TESTING SYSTEMS}

Original thoughts were that the IC is a maximum of the system and that AP and HF will decrease the reliability determined by the physics of the equipment. A more realistic view is that the modules are not independent and to ease the investigation of the system, one can investigate modules separately, but to have the final judgement on the NDT system, all modules have to be scrutinized together. This is illustrated schematically in figure 2.1. The formula for overall reliability can be written as

$$
\mathcal{R}=\mathcal{F}[f(I C), g(A P), h(H F)]
$$

where $\mathcal{R}$ is an overall reliability of the system and $f(I C), g(A P)$ and $h(H F)$ are a functions of intrinsic capability, application parameters and human factors, respectively.

\subsection{Signal detection}

As seen in section 1.1.3, one of the most important functions of the NDT system is to indicate the presence of flaws in the inspected object. The apparatus applies a stimulus to the inspected object and a response to the stimulus (signal) is observed. The system attempts to detect signals coming from a flaw in a background of interference or noise [67]. A decision (diagnosis) if the signal (flaw) is present or not has to be made based on the interpretation of the signal in frequently ambiguous situations [68. It is not a problem to make the decision when the alternatives are obvious and the evidence is clear. But data are usually insufficient for certainty or complete accuracy of the diagnosis. Signal-detection theory gives a general framework to describe and study decisions.

The ambiguity of the signal detection is illustrated in figure 2.2. Three ultrasonic A-scans at three different positions are shown. The signal amplitude at the position 1 is much higher than the surrounding noise and a positive decision about the presence of the signal is easy. At the position 2 the signal amplitude is smaller but still large enough to be distinguished from the surrounding noise. The most difficult situation is illustrated at position 3. The signal is still somewhat larger than the surrounding noise, but the situation is ambiguous. It depends on the inspector if this will be treated as the signal or not. To make decisions consistent and objective, a decision threshold or decision criterion needs to be set. Every amplitude larger than some specific value will be proclaimed positive and every amplitude smaller than this value as negative. In 

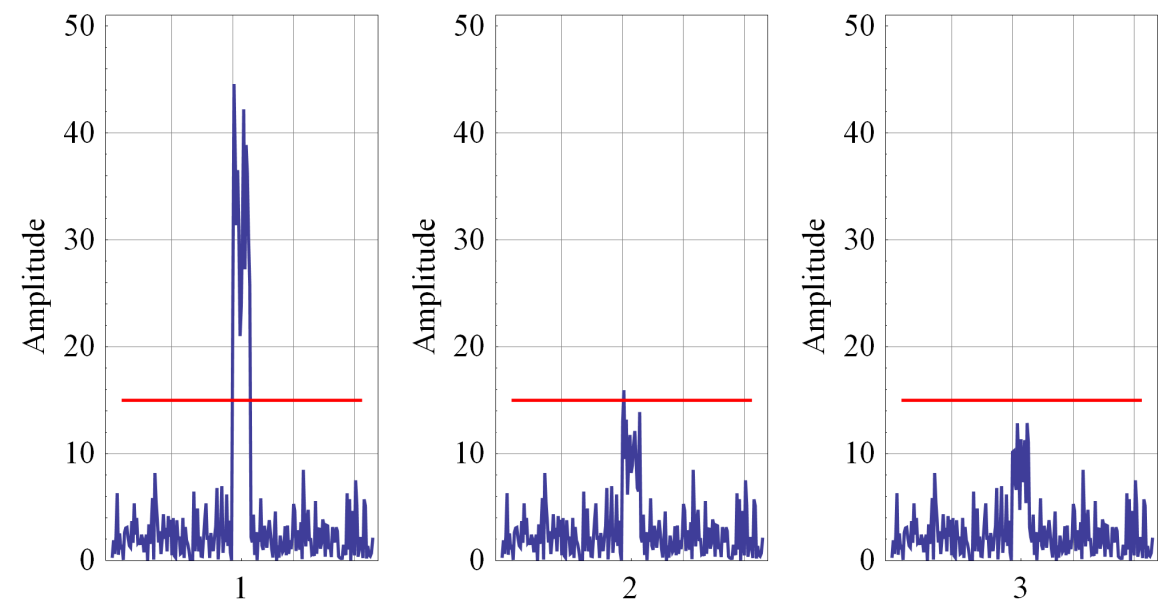

Figure 2.2: Signal detection in the ultrasonic A-scan - Three inspection situations with different signal strengths in a background of a noise with indicated decision threshold

figure 2.2 the threshold is illustrated with the horizontal red line. For all amplitudes higher than the threshold, a presence of a signal is registered. In the given example, there is a presence of a signal at the positions one and two, whereas at the position 3 there is no presence of a signal.

When making a decision in two-alternate decision tasks, there are four possible outcomes. The object under study can have one of two possible states. Either there is a condition (flaw) present (boolean true state, 1) or there is none (boolean false state, 0 ). The inspector can make one of the two possible decisions. Either there is a condition present (positive decision) or there is none (negative decision). Intersections of these two make a 2 x 2 matrix of possible outcomes as shown in table 2.1. Truepositive (TP) is the outcome where the inspector makes a positive decision and there is a flaw present in the object. True-negative (TN) is an outcome where the inspector decides that there is no flaw present and that is also the case. These two outcomes are wanted (correct) ones. On the other hand, if the inspector makes a decision that there is no flaw present, when there is in fact one (false-negative, FN), or, when he makes a decision that there is a flaw when there is none present (false-positive, FP), he is producing outcomes that are not wanted (incorrect). In the language of signal detection, terms: hit, correct rejection, false alarm and miss are also used for: true- 


\section{STATE-OF-THE-ART IN RELIABILITY OF NON-DESTRUCTIVE TESTING SYSTEMS}

\begin{tabular}{|c|c|c|c|}
\hline & & \multicolumn{2}{|c|}{$\begin{array}{l}\text { State of the object } \\
\text { (Flaw present?) }\end{array}$} \\
\hline & & True & False \\
\hline \multirow{3}{*}{$\begin{array}{c}\text { Inspector's } \\
\text { Decision }\end{array}$} & Positive & $\mathrm{TP}$ & $\mathrm{FP}$ \\
\hline & & (hit) & (false alarm) \\
\hline & Negative & $\begin{array}{l}\text { FN } \\
\text { (miss) }\end{array}$ & $\begin{array}{c}\mathrm{TN} \\
\text { (correct rejection }\end{array}$ \\
\hline
\end{tabular}

Table 2.1: Matrix of stimuli and responses in two-alternate decision task

positive, true-negative, false-positive and false-negative, respectively.

It is important to notice that every system can achieve any desired TP rate by either decreasing the decision threshold or by increasing the amplification of the signal (and noise). The adverse effect of this is that the FP rate will also increase, because more of the noise will be treated as a signal. The opposite procedure can be performed to decrease the FP rate. By increasing the decision threshold or decreasing the sensitivity of the apparatus, the FP can effectively be brought to zero. However, TP will also decrease. The decision threshold value is chosen according to the cost associated with correct and incorrect decisions of both kinds. All this make it obvious that it is not possible to compare two inspection systems judging only by the TP, because every system can easily achieve the desired TP level, but with the increase of FP.

\subsection{Receiver operating characteristic (ROC)}

By performing trials on the test objects with known flaws (i.e. states), conditional probabilities of inspectors response given the state of the object, can be assigned. For example, $P$ (positive|true) denotes conditional probability that the inspector will make a positive decision about the presence of the flaw when, there is indeed a flaw present in the object under study. Since the inspector has to make a decision - either there is or there is no flaw present, the columns of the matrix sum up to one:

$$
P(\text { positive } \mid \text { true })+P(\text { negative } \mid \text { true })=1
$$

and 


$$
P(\text { positive } \mid \text { false })+P(\text { negative } \mid \text { false })=1
$$

It follows that there are only two independent probabilities in the matrix. Therefore it is possible to express system performance by only two probabilities. The ROC analysis presents the true-positive (hit) probability $P(T P)$ plotted against false-positive (false alarm) probability $P(F P)$. At the same time this also represent $1-P(F N)$ plotted against $1-P(T N)$.
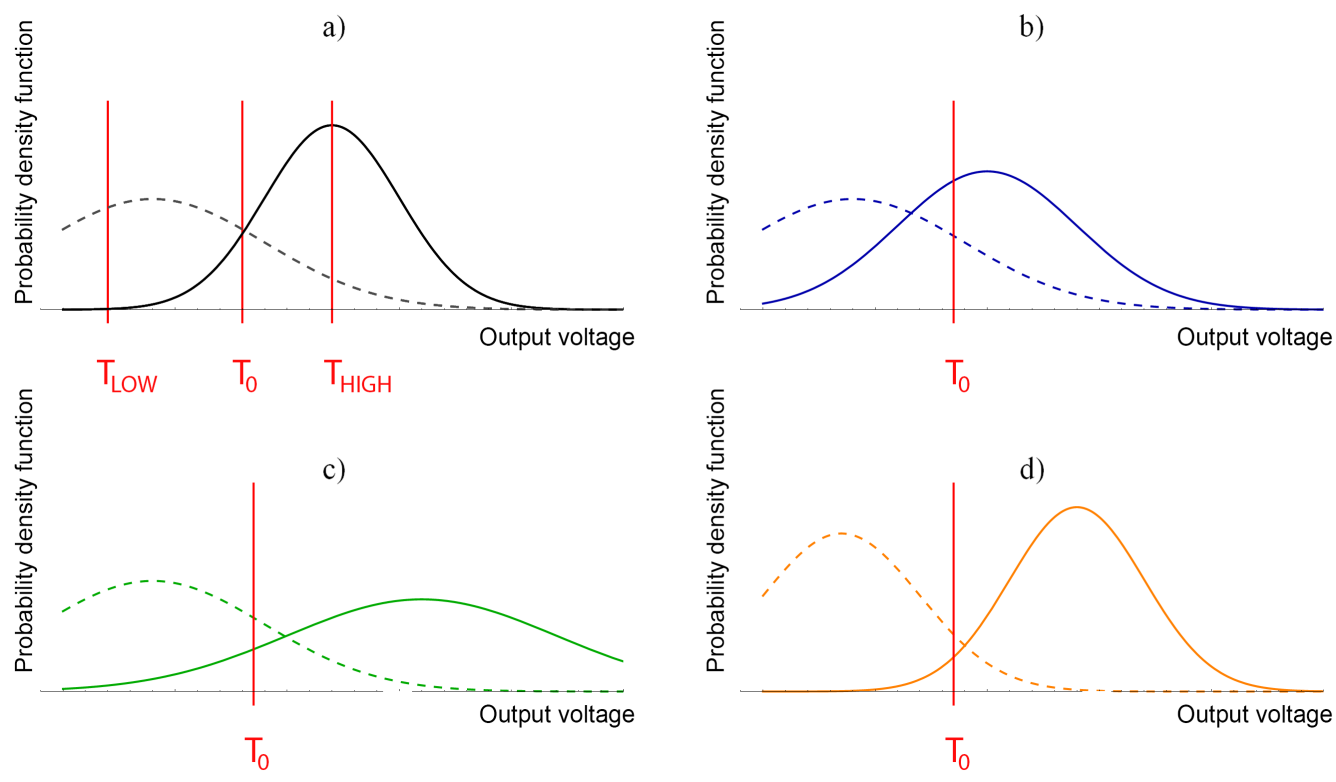

Figure 2.3: Signal and noise probability distributions - Four NDT systems with different signal (solid line) and noise (dashed line) probability distributions with indicated decision threshold(s) (red line)

The diagram in figure 2.3 a) shows the signal (solid line) and noise (dashed line) probability distributions of one NDT system, with indicated three different decision thresholds: reference threshold $T_{0}$, lower threshold $T_{L O W}$ and higher threshold $T_{H I G H}$.

Only signals that are higher than the threshold will be treated as detection, whereas signals lower than the threshold will be false negatives. The noise lower than the decision threshold will be treated as a true negative, whereas noise higher than the threshold will be treated as a false positive. Figure 2.4 shows the ROC curve of the same system with indicated three operating points, based on these three thresholds. 


\section{STATE-OF-THE-ART IN RELIABILITY OF NON-DESTRUCTIVE TESTING SYSTEMS}

As it can be seen, the choice of the threshold determines both the $P(T P)$ and the $P(F P)$ of the system. Decreasing the threshold from the reference threshold $T_{0}$ to the lower threshold $T_{L O W}$, the operating point moves along the curve up, increasing the $P(T P)$ and $P(F P)$. Increasing the threshold from the reference threshold $T_{0}$ to the higher threshold $T_{H I G H}$, the operating point moves along the curve down, decreasing the $P(T P)$ and $P(F P)$.

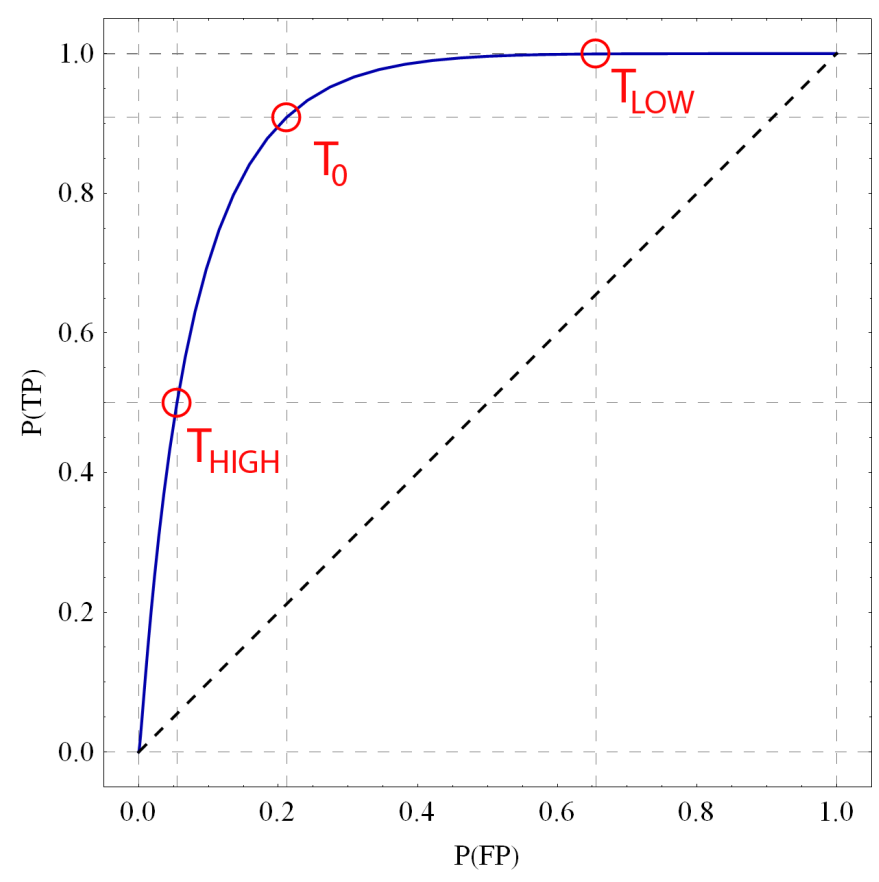

Figure 2.4: Receiver operating characteristics (ROC) curve - Red circles indicate three operating points based on different thresholds $T_{0}, T_{L O W}, T_{H I G H}$, as shown in figure 2.3 a).

Diagrams in figure $2.3 \mathrm{~b}$ ), c) and d) show three systems with different discrimination aspects or accuracy. Different accuracy is evident by different signal and noise probability distributions spread and separation. The same decision threshold $T_{0}$ is used for all three systems. Figure 2.5 shows ROC curves of those three systems with indicated operating point based on the chosen decision threshold $T_{0}$. The best performance demonstrates the system of discrimination aspect shown in figure $2.3 \mathrm{~d}$ ). It shows the highest $P(T P)$ while having the smallest $P(F P)$. The orange curve in figure 2.5 is the ROC curve of this system. The system shown in figure $2.3 \mathrm{c}$ ) is better than the one shown in figure 2.3 b) because it demonstrates higher $P(T P)$ while having the same 
$P(F P)$. The ROC curves of these two systems are shown in figure 2.5 in green and blue, respectively.

As already noted, the ROC curves are calculated for only one size of the flaw. To be able to establish if the NDT system is capable of finding flaws that could undermine the structural integrity, it is necessary to know the capability of the NDT system to detect flaws regarding a flaw size. This is achieved by expressing the POD as function of the flaw size. The POD diagram will be presented in the following section.

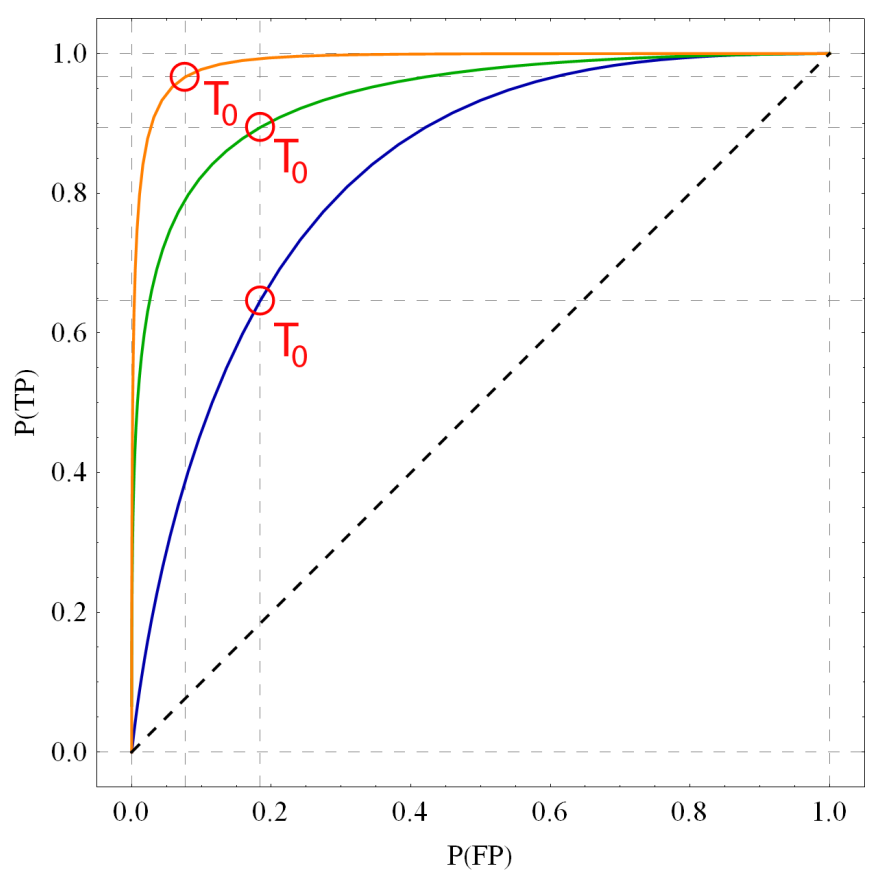

Figure 2.5: Comparison of ROC curves - ROC curves from three different systems shown in figure 2.3 b) (blue curve), c) (green curve) and d) (orange curve), with the operating point based on the same threshold

\subsection{Probability of detection (POD) curve}

It has been shown in the previous section that the conditional probability $P$ (positive|true) (or $P(T P)$ or probability of detection (POD)) is not enough to compare different inspection systems, because any system can achieve the desired level of POD by increasing the sensitivity or by decreasing the decision threshold. Nevertheless, the diagram showing the POD expressed as function of the flaw size - the POD curve - is frequently used 


\section{STATE-OF-THE-ART IN RELIABILITY OF NON-DESTRUCTIVE TESTING SYSTEMS}

to show the capability of the NDT systems to detect flaws regarding their size. The POD curve can be used for comparison of different systems if the same decision threshold is used. For example, a common procedure in ultrasonic inspection is to consider amplitudes that are three times larger than the surrounding noise (signal-to-noise ratio $(\mathrm{SNR})=3)$ as a signal. One of the big advantages of the POD curve over the ROC diagram is that the performance of the NDT system as a function of the flaw size is given, whereas the ROC curve is valid only for one size of the flaw.

POD is estimated through inspection experiments on specimens containing flaws of a known size. Data is recorded either as a hit/miss or as a signal response. In a hit/miss analysis, for each flaw it is recorded if the flaw was detected (a hit) or not (a miss). In a signal response analysis, data is recorded as magnitude of the response signal from the flaw. When a signal exceeds the defined decision threshold, it is counted as a detection [69]. Single most influential factor in most of the cases is the target size [6]. The influence of other factors on the POD is also acknowledged [70]. The POD has been calculated as a function of the e.g. crack inclination angle [71].

Modern inspection systems are able record the magnitude of the response signal. There is more information about the POD of a flaw contained in signal response data than in hit/miss data, because not only exceeding the decision threshold, but also the amount by which it is exceeded, will reflect on the POD. If the flaw size is denoted by $a$, the magnitude of the signal can be seen as the perceived flaw size and is denoted by $\hat{a}$. It is anticipated that the signal magnitude will depend on the flaw size [73]. Review of the data from some experimental studies shows a linear relationship on a log-log scale [72], although it has been recognized that the linear model is not the most effective in all applications and some new approaches have been proposed [74. A linear relationship between the response signal magnitude and the flaw size can be observed if plotted in the $\hat{a}$ versus $a$ diagram. Data from [72] of the eddy-current inspection of surface cracks is shown in figure 2.6. The linear model is expressed by:

$$
\ln \hat{a}=A_{0}+A_{1} \ln a+\delta
$$

where the coefficients $A_{0}$ and $A_{1}$ can be determined by the maximum likelihood method. The variance of the measurement is denoted $\delta$ which is assumed independent of the flaw size, normally distributed with the zero mean and standard deviation $\sigma_{\delta}$ : 


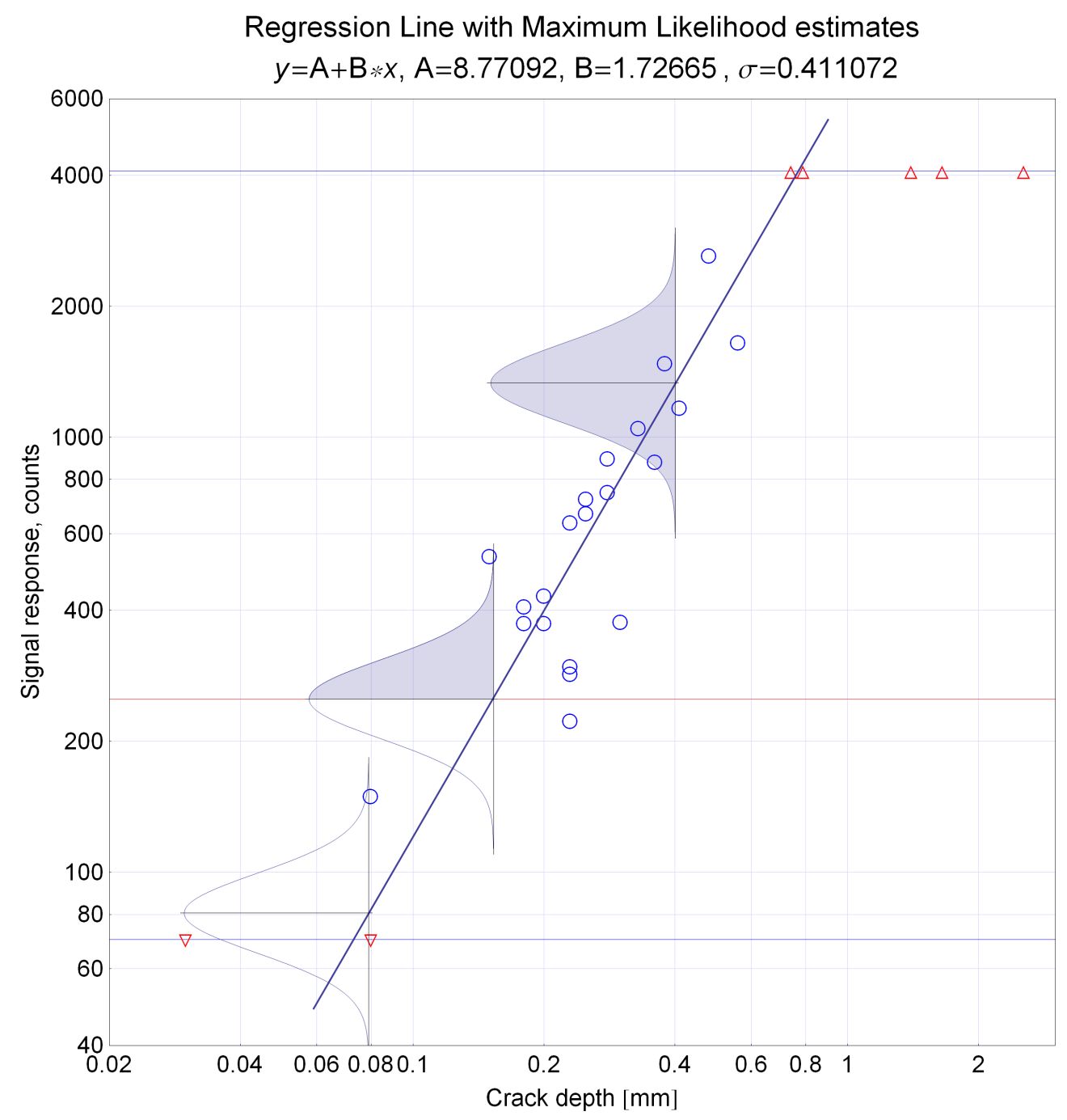

Figure 2.6: $\hat{a}-a$ diagram - with indicated probability density function. Eddy-current inspection data from [72] 


\section{STATE-OF-THE-ART IN RELIABILITY OF NON-DESTRUCTIVE TESTING SYSTEMS}

$$
\delta \sim \mathcal{N}\left(0, \sigma_{\delta}\right)
$$

where $\mathcal{N}$ is a normal distribution function.

Equation 2.4 can be seen as an intrinsic capability of the NDT system that represents a mean system performance whereas application parameters and human factors are the cause of the variance in the measurement [75]. The standard deviation $\sigma_{\delta}$ can be determined from the residuals of the experimental data from the linear regression [76]. POD of the flaw of the size $a$ is calculated as probability that the recorded signal lies above the decision threshold $\hat{a}_{d e c}$ :

$$
P O D(a)=1-\Phi\left[\frac{\ln \hat{a}_{d e c}-\left(A_{0}+A_{1} \ln a\right)}{\sigma_{\delta}}\right]
$$

where $\Phi$ is standard normal distribution function.

To indicate the reliability of the estimate of parameters of the model, the lower $95 \%$ confidence interval is placed on the $\operatorname{POD}(a)$ curve [77, 78]. The point where the lower $95 \%$ confidence band intersects $90 \%$ detection probability is denoted as $a_{90 / 95}$ point. It indicates the flaw size that can be detected with $90 \%$ probability in $95 \%$ of cases. This point can be used to describe the NDT system detection capability. It indicates the size of the flaw that would still be reliably detected by the NDT system, based on the safety level accepted by the airworthiness authorities [79].

Figure 2.7 shows a typical POD curve (solid curve), calculated from the data in [72]. A lower $95 \%$ confidence band is also plotted (dashed cure) with indicated $a_{90 / 95}$ point; a point where the lower $95 \%$ confidence band intersects $90 \%$ probability of detection.

Even if it is considered a standard tool for quantifying an inspection system's detection capability, it is believed that POD curves are not very well understood by many who use and apply them 66. POD curves calculated for one specific combination of the NDT system, material and geometry were used for other applications, even for other NDT methods. Probably one of the most misunderstood element of the POD analysis is the influence of the sample size on the shape of the POD curve and the confidence interval [80]. 


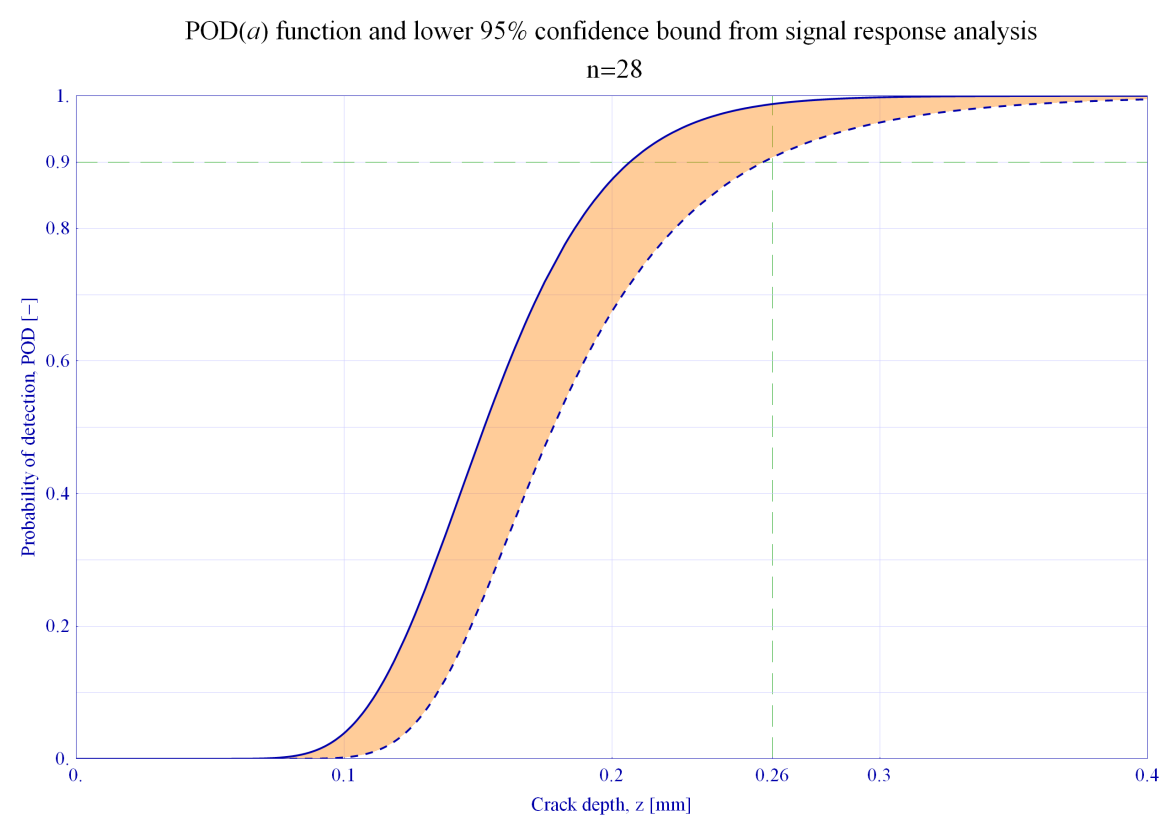

Figure 2.7: Probability of detection (POD) curve - eddy current inspection data from 72

\section{Influence of the sample size and scatter of the data on the POD esti- mation}

A special care has to be taken when deciding the sample size for the experiment and how good this sample represents the population of the flaws. The sample size influences the confidence in the POD estimation. The smaller the size of the sample, the larger the width of the confidence interval will be. The wider the confidence interval, the smaller the confidence in the POD estimation. Width of the confidence interval has a direct influence on the location of the $a_{90 / 95}$ point which is often used as a measure of the capability of the NDT system to detect flaws. The scatter of the data around the regression line will influence the width of the region where the POD curve changes from 0 to 1.

These influences will be demonstrated on an example with a computer generated data. Some linear relationship between the signal and the flaw size is assumed, so that the signal increases with the increased flaw size. Assuming the underlying normal distribution (population), sample of 100 points with zero mean and standard deviation $\sigma_{1}=\sigma_{0}$ was generated. Two another samples also with 100 points each, but with 


\section{STATE-OF-THE-ART IN RELIABILITY OF NON-DESTRUCTIVE TESTING SYSTEMS}

standard deviations $\sigma_{2}=1.5 \sigma_{0}$ and $\sigma_{3}=3 \sigma_{0}$ were also generated. From each of these three samples, 50 points were randomly selected in the next step. From these samples of 50 points, 20 points were randomly selected. Finally, from the samples of 20 points, samples were made by randomly selecting 10 points. All these operations resulted in 12 samples. These samples are shown in $12 \hat{a}$ versus $a$ diagrams in figure 2.8 . In the first column samples with the scatter $\sigma_{0}$ are shown. In the second and the third column samples with the scatter $1.5 \sigma_{0}$ and $3 \sigma_{0}$ are shown respectively. In the first row, samples with 10 data points are shown. In the second, third and fourth row, samples with 20,50 and 100 data points are shown respectively. In all diagrams, the regression line is also plotted. It can be observed that the scatter around the regression line is increasing in diagrams from left to right and that the number of data points is increasing in diagrams from top to bottom. A deviation of the regression line from diagram to diagram can also be observed, but since all samples are relatively good representatives of the population, these deviations are small. The diagram in the lower left corner represents the sample with the most data points and the smallest scatter so it is the sample that best represents the population.

For each of these 12 samples shown, POD was calculated. The POD curves are shown in figure 2.9. Again, first, second and third column, show POD curves from samples with $\sigma_{0}, 1.5 \sigma_{0}$ and $3 \sigma_{0}$ respectively. First, second, third and fourth row show POD curves for the samples with 10, 20, 50 and 100 data points, respectively. It can be observed that as the scatter of the data increases in diagrams from left to right, the transition region of the POD from 0 to 1 is becoming wider. The width of this region is approximately $0.25 \mathrm{~mm}$ for the samples with the scatter $\sigma_{0}$ and more than $0.6 \mathrm{~mm}$ for the samples with the scatter $3 \sigma_{0}$. Also, the width of the confidence interval (the distance of lower 95\% confidence bound from the POD curve) is also increasing as the $\sigma$ increases. As the number of data points in the samples increases, it can be observed that the width of the confidence interval is decreasing. For the largest sample with the smallest scatter, in diagram in the lower left corner, the lower confidence band is very close to the POD curve.

The $a_{90 / 95}$ frequently used as a measure of the systems detection capability is changing accordingly. For the small sample sizes and for the large scatter of the data points, it might not even be possible to determine it. 

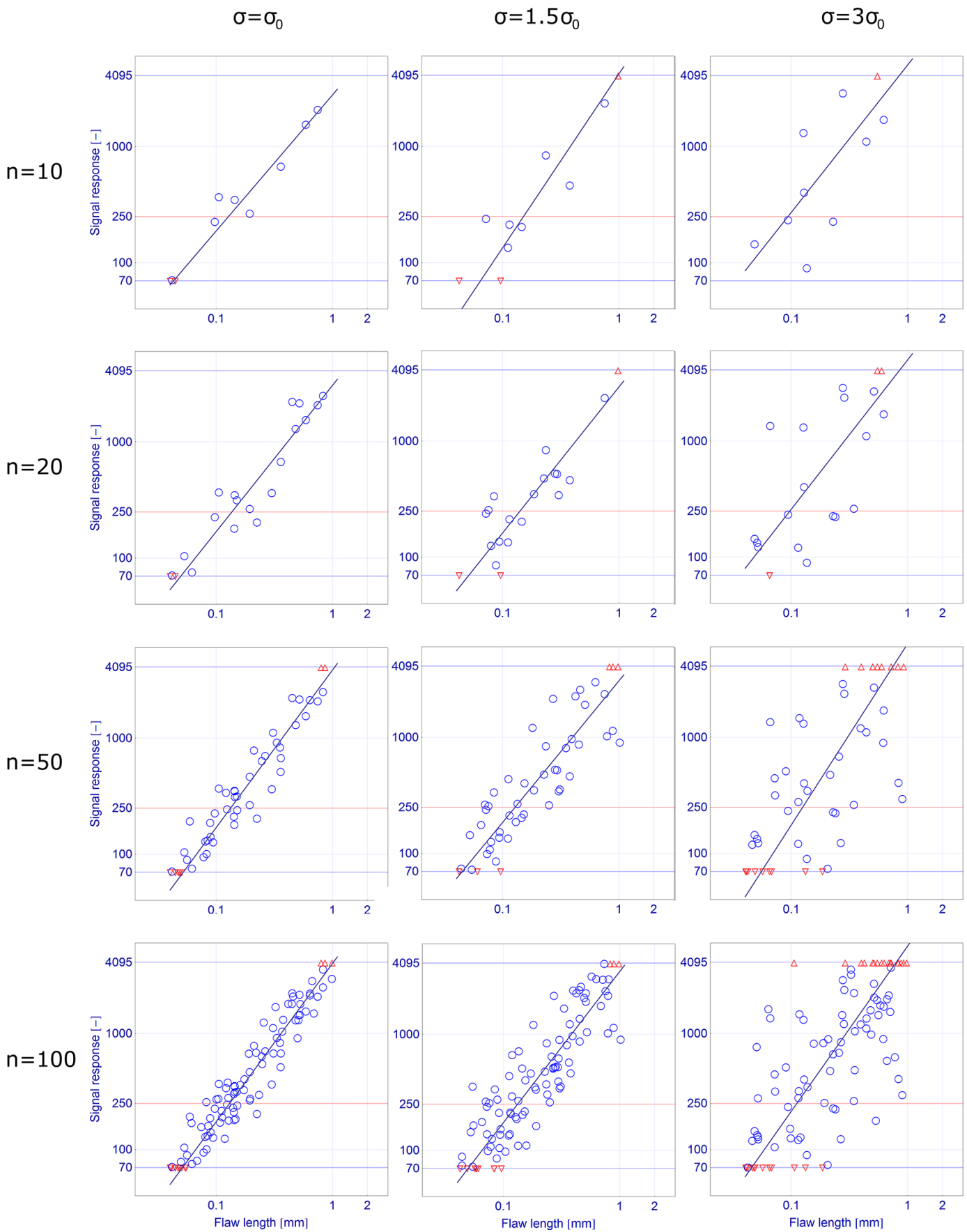

Figure 2.8: Comparison of $\hat{a}-a$ diagrams - with different sample sizes and different scatter of the data 


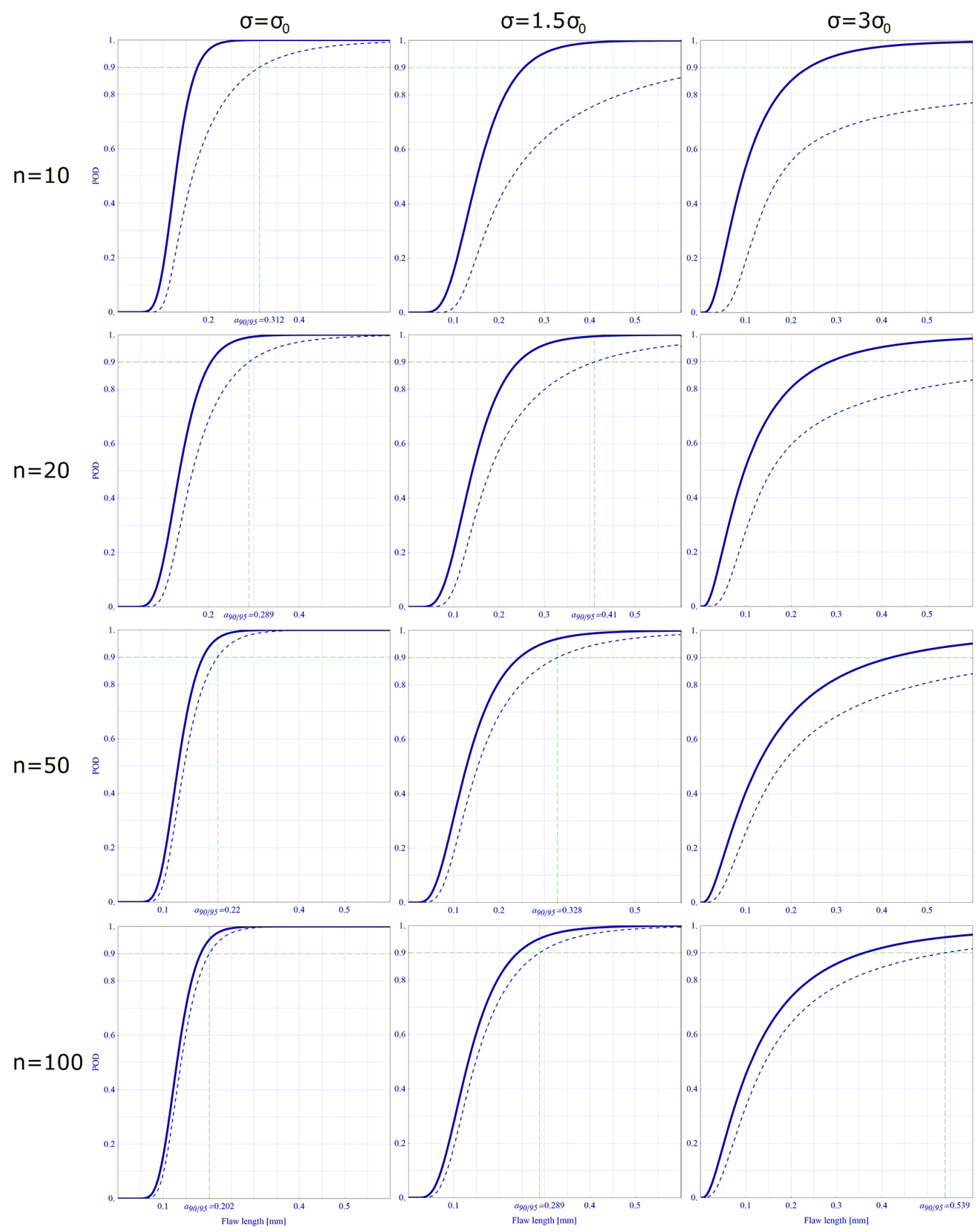

Figure 2.9: Comparison of POD curves - with different sample sizes and different scatter of the data 


\subsection{Computer simulation in NDT}

New demands on reliability of used NDT procedures and methods have stimulated the development of simulation tools for NDT. To qualify inspection procedures, extensive experimental work is normally required. A validated model of the inspection process has the ability to be an alternative and a complement to the experimental work in order to reduce costs. The most significant advantage of a validated and verified computer model is possibility in parametric studies leading to the generation of new testing procedures [81]. Computer simulation of inspection simplifies the process, improves the understanding of the phenomena and provides a tool that helps the analysis and interpretation of inspection results.

There has also been significant work done in the reliability community over the years to incorporate physics-based models of the inspection process into the POD estimation [82]. A number of models are available for POD using a range of approaches. These models are being increasingly used and validated [83].

The ultrasonic simulation software used for the POD analysis in this thesis has been developed at the Federal Institute for Material Research and Testing (BAM), division 8.4 Acoustic and Electrical Methods. Software was often used successfully for the probe design and the steering parameter optimisation. The programs for sound field and echo calculations use the point source synthesis (PSS) method taking into account the elastodynamic behaviour at the material boundaries [84, 85, 86]. The physical background of the method is the Huygens principle where each point of a wave front is the starting point of an elementary wave and the new wave front is obtained as the superposition of all elementary waves. Surfaces of the modelled transducers and reflectors are divided into a number of small patches, which are mathematically represented by point sources. The maximum size of the patches depends on the allowed phase shift between neighbouring patches (a small part of the wavelength, e.g. $\lambda / 7$ ) in relation to the observation point. The elementary sound fields created by the point sources and described as the far fields of the point sources are superposed at the observation point considering Snell's law at material interfaces, which can be flat or curved. The contributions of all point sources are additionally multiplied with the corresponding directivities.

The simulation software is developed for one or two dimensional phased array probes and for contact or immersion technique on plane or cylindrical surfaces. The result is 
the propagation of an acoustic pulse in time and space of longitudinal and/or transversal waves taking into account the geometry, the time delay distribution (focal law), the sound velocities, mode conversion factors, and attenuations in the wedge and the modelled material. The method calculates diffraction phenomena correctly, especially for the side and the grating lobes considering the geometry of the single element and the whole probe. Therefore the calculation can also be applied to short distances from the probe in the near field. The dynamic focusing technique applied with the phased array ultrasonic probes by SKB, has been implemented specially for this project [87]. 


\section{3}

\section{Problem Statement}

It has been emphasized that it is important to determine a reliability of the NDT system in applications where a flaw that is not detected could lead to catastrophic consequences. It has been also highlighted that the POD curve is a standard tool for quantification of NDT reliability and that it is commonly plotted as a function of the flaw size. In many applications this is sufficient, since the size of the flaw is both a principal factor that determines its severity for the structural integrity and a principal factor that determines the POD of the flaw. When the NDT reliability project of the Swedish final repository for spent nuclear fuel began and first ultrasonic inspection data started to be analysed, several problems have been encountered.

First, dimensions of the acceptable flaw were calculated with the damage tolerance analysis of the cast iron insert and specified with two parameters: flaw length and flaw depth extension. Since a conventional POD curve is expressed as a function of one parameter it was not clear to which parameter to compare the POD to determine if the NDT system is capable of detecting critical flaws. Also, the analysis of the data from the ultrasonic inspection of copper tube and lid showed high scatter of the response signal amplitudes. This indicated that there are some other factors that have a strong influence on the POD beside the flaw size. Also, there was no evident linearity between the response signal amplitude and the size of the flaw, thus basic requirements for the conventional signal response analysis were not met.

Secondly, inspection of the copper tube revealed a large variation in ultrasonic attenuation from location to location of the tube. It is known that ultrasonic attenuation 


\section{PROBLEM STATEMENT}

influences the POD of the flaw, so a large variation of attenuation across the component will cause a large variation of the POD. This problem needed to be addressed too.

Third problem that was encountered was associated with the number of inspections. Due to the relative geometrical complexity of the components, their thickness and ultrasonic attenuation in the material, components need to be inspected with several ultrasonic inspections to fulfil the requirement to inspect $100 \%$ of the volume of the canisters. Since the conventional POD analysis gives the POD of one inspection, it is not known what is the overall POD when several inspections are used in conjunction.

\subsection{Influence of multiple parameters on the flaw severity and on the POD}

In the final repository, the canister will be exposed to compression from the hydrostatic pressure and the swelling pressure from the surrounding bentonite. During the extreme long time period an ice-sheet of $3 \mathrm{~km}$ is expected to form during the ice age. When the ice starts to melt, there is a possibility of an earthquake induced rock shear through a deposition hole. The effect on the canister by such a shear load has been investigated [88]. Damage tolerance analysis has been performed with the aim to calculate acceptable flaw sizes with regard to safety margins against fracture [52]. As the most severe flaw geometry a surface-breaking, semi-elliptical surface crack-like flaw was identified. The flaw is illustrated in figure 3.1. The location where the max principal stress is the highest was identified for the rock shear perpendicular to the axis of the canister, hitting the canister at $3 / 4$ of the height from the bottom. The location of the max principal stress is illustrated in figure 3.2 . For a flaw located in the zone of max principal stress and a $5 \mathrm{~cm}$ shear, acceptable flaws are calculated and shown in table 3.1 .

As it can be seen from the table, the size of the acceptable flaw is given by two dimensions: its length and depth extension. It is common that the minimum flaw size that can be detected by the NDT system serves as an input for the design of the component when calculating the maximum allowable flaw size. In this project an inverse approach was used. Due to the long duration of the project (over one decade) it was assumed that the NDT technology will improve significantly before the repository starts to operate. Instead of limiting the design of the canister with the capability of 


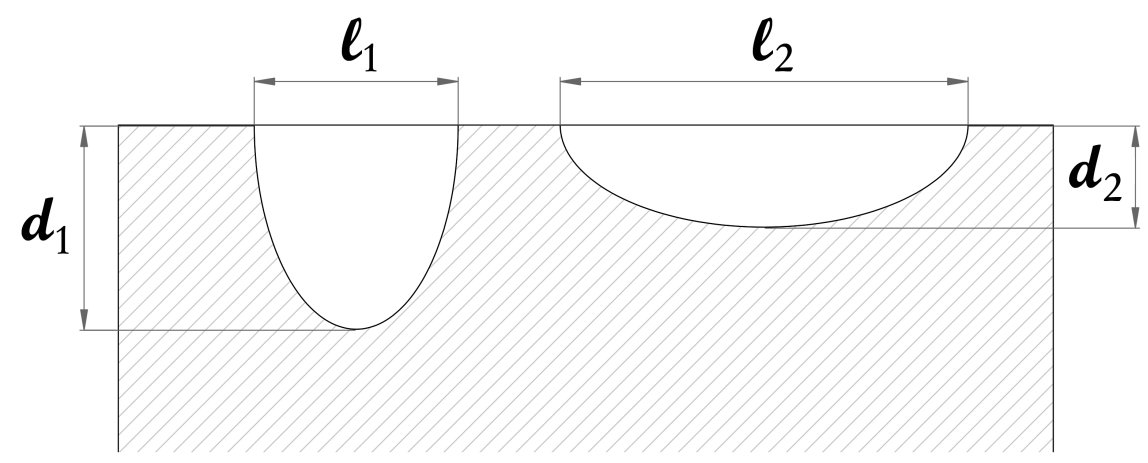

Figure 3.1: Semi-elliptical, surface breaking, crack-like flaw - The most severe flaw in the shear loading case of the canister

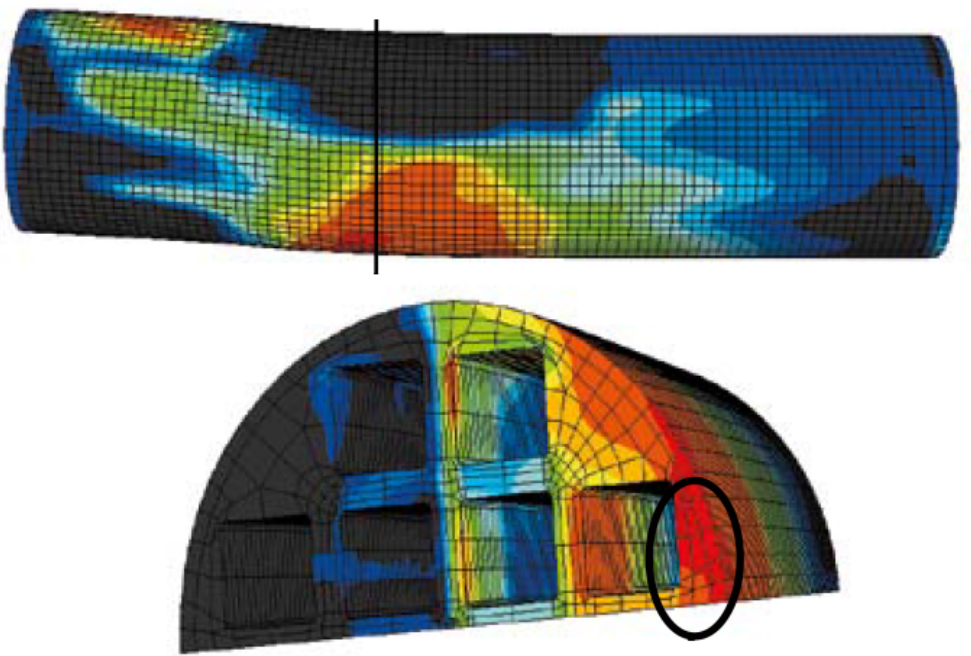

Figure 3.2: Location of max principal stress - Placement of the most severe flaw in maximal principal stress zone (red) for calculation of the acceptable flaw size (Illustration from [52]) 


\section{PROBLEM STATEMENT}

\begin{tabular}{cccc}
$\begin{array}{c}\text { Density of bentonite } \\
\rho\left[\mathrm{kg} / \mathrm{m}^{3}\right]\end{array}$ & $\begin{array}{c}\text { Acceptable depth } \\
d[\mathrm{~mm}]\end{array}$ & $\begin{array}{c}\text { Acceptable length } \\
l[\mathrm{~mm}]\end{array}$ & $\begin{array}{c}\text { Area } \\
A=1 / 4 \pi d l\left[\mathrm{~mm}^{2}\right]\end{array}$ \\
\hline 2050 & 4.5 & 27 & 95 \\
2000 & 8.7 & 52.2 & 357 \\
1950 & $>10$ & $>60$ & $>471$ \\
\hline
\end{tabular}

Table 3.1: Acceptable semi-elliptical crack dimensions located in the zone of maximum principal stress for a $5 \mathrm{~cm}$ shear

present NDT systems, the NDT system that will be used for the inspection, needs to demonstrate that it is capable to reliably detect all critical flaws that can occur in the material. As previously shown, the capability of the NDT system is quantified through the POD curve. Since the POD curve is calculated as a function of one parameter usually the flaw size - it is not clear to which of the two acceptable flaw dimensions POD should be calculated to determine if the NDT system is capable to detect the flaw. One option is to make the POD curve for each of those two parameters. This requires a large amount of flaws to inspect in reliability study, which is time-consuming and expensive. Another option would be to calculate the area of the flaw and express the POD as a function of the flaw area. This could however lead to either overestimation or underestimation of the NDT system's capability to detect flaws. Obviously, there is a need to express the POD as a function of more than one parameter.

When the inspection data from the thick canister copper components were evaluated, it has been observed that there is a large scatter in the $\hat{a}$ versus $a$ diagram and that the linearity between the response signal amplitude and flaw size is not quite obvious. The amplitude of the response signal must be influenced by other factors beside the size of the flaw. To perform a valid reliability estimation of the NDT system, these parameters need to be identified and the POD has to be calculated as a function of these parameters. Again, there is a need to express the POD as a function of more than one parameter.

\subsection{Ultrasonic attenuation and its influence on the POD}

When inspecting relatively thin components where the path that the wave travels is short or when inspecting materials with low attenuation, the amplitude of the wave does 
not change considerably. Since the influence on the amplitude is negligible, neither the attenuation nor the distance of the flaw from the sensor will be essential influencing parameters on the POD.

The thickness of $5 \mathrm{~cm}$ of the canister tube and the scatter of the wave on the grain boundaries in copper cause a considerable change in the amplitude as the wave propagates through the material. Furthermore, large variation in attenuation across the almost $5 \mathrm{~m}$ long copper tube has been observed when the back-wall echoes in the ultrasonic C-scan were evaluated. The ultrasonic C-scan of the whole tube is shown in figure 3.3. It is known that the attenuation has an influence on the POD of the flaw. The flaws with the same geometry will have different POD depending on their location in the tube, i.e. depending on the micro-structure of the surrounding material. Therefore, the POD should be calculated as a function of attenuation and distance from the inspection surface in order to properly estimate the POD of the flaw in the thick copper component.

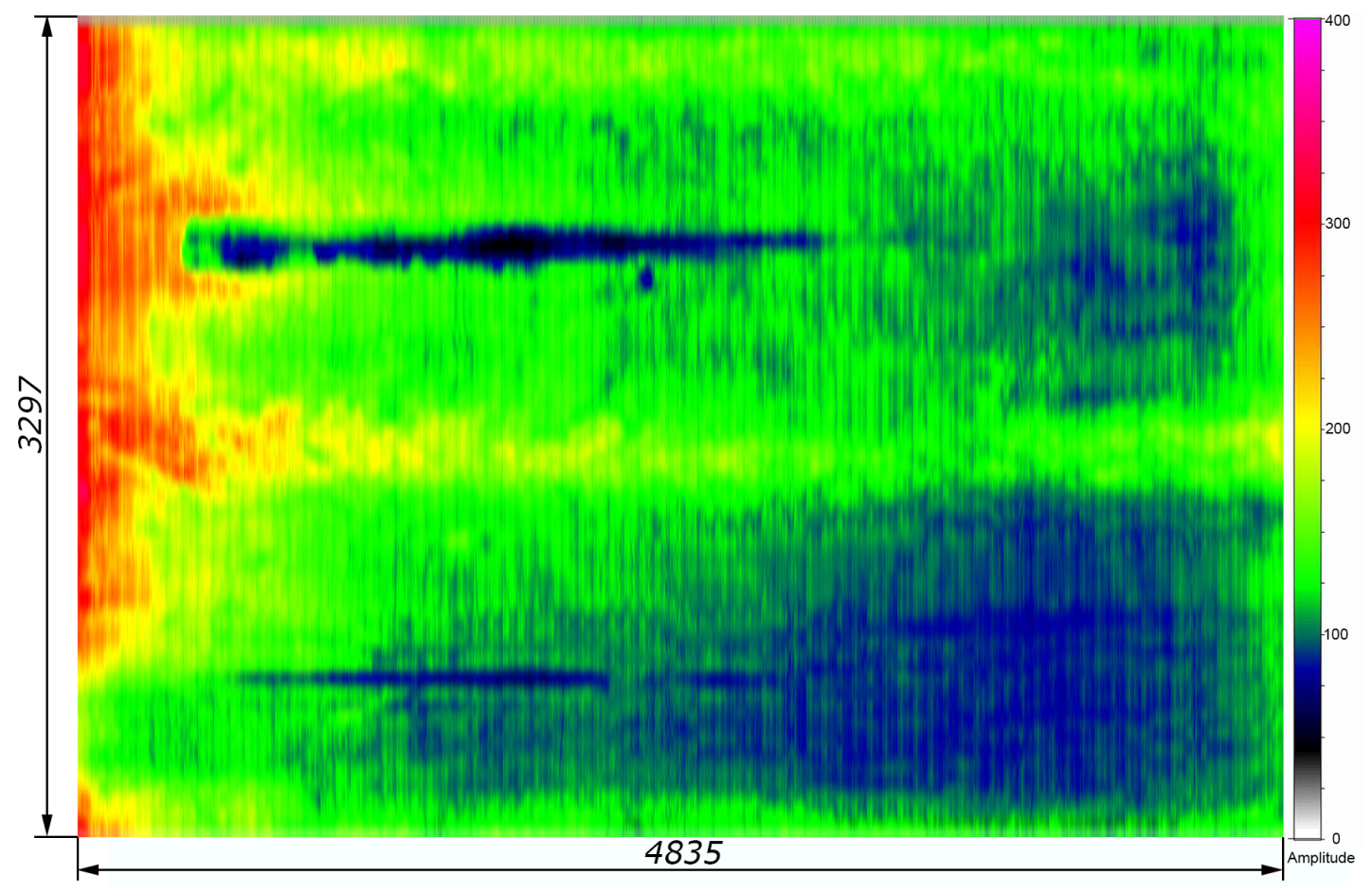

Figure 3.3: C-scan of the whole tube - Variation of the back-wall echo amplitude along the almost $5 \mathrm{~m}$ length of the tube. The length of the tube is plotted on a horizontal axis and circumference on a vertical axis. 


\section{PROBLEM STATEMENT}

\subsection{Multiple inspections of a component and an overall POD}

A location of the flaw in the component can play an important role when determining flaw severity for the structural integrity. It has also been pointed out that when thick components with high attenuation are inspected, the depth of the flaw can have an important influence on the POD. Therefore it is necessary to express the POD as a function of the depth. A conventional POD analysis gives the capability of the NDT system to detect flaws with one value (e.g. $a_{90 / 95}$ ), but when the position of the flaw is an influencing parameter, it would be much easier to estimate the capability of the NDT system to detect flaws if the POD would be shown as a 3-D scatter plot over the geometry of the component.

NDT methods and techniques present different advantages and limitations. Due to these differences and especially when inspecting complex geometries [89] or looking for different types of flaws, the use of more than one method or technique is required to inspect material fully [90]. With the conventional POD analysis, only the POD of individual inspections can be determined. Data originating from different sensors can be conflicting, incomplete or unclear. Only by performing data fusion and creating the overall POD, the benefit of additional inspections can be seen [91] and the POD correctly estimated. Data fusion can be done in many ways and on many levels [92]. There is also a possibility to assign weight to different sensor depending on the level of thrust 93. The overall POD diagram can then also be used to optimize the inspection system [94]. Relative complex geometry of the copper lid of the canister, coupled with high attenuation in copper, demands that the component is expected with several inspections to fulfil the requirement to inspect $100 \%$ of the volume of the component.

The NDT system designers preliminary planned 10 different inspection for the inspection of the canister lid. The inspection set-ups are illustrated in figure 3.4. The inspections were planned according to the best practices of ultrasonic inspection. In order to cover the complete volume, the lid is to be inspected both from the axial and the radial surface, using contact and immersion ultrasonic techniques. The overall POD needed to be calculated, to make sure that the whole volume of the component is inspected with sufficiently high POD, when using many different inspection set-ups. 
To calculate the overall POD, the data originating from different inspections need to be combined.

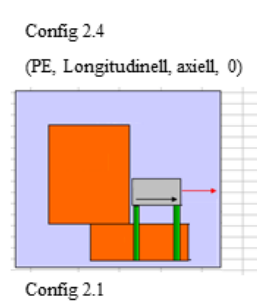

( $\mathrm{PE}$, Longitudinell, axiell, 0)

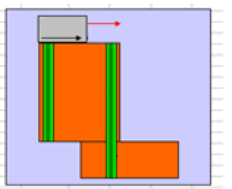

Config 2.12

(PE, Transversell, radiell, +45 )

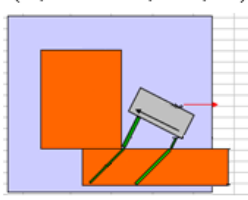

Config 2.5

(PE, Longitudinell, axiell, 0)

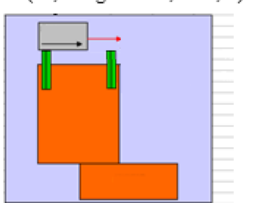

Config 2.13

(PE, Transversell, radiell, -45 )

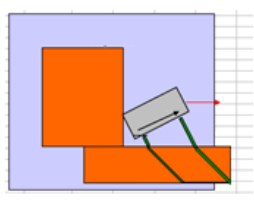

Config 2.2

(PE, Longitudinell, radiell, 0)

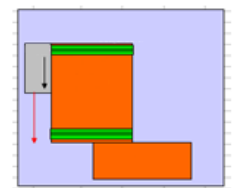

Config 2.14

(PE, Transversell, tang, +45)

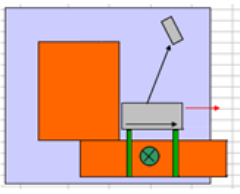

Config 2.3

(PE, Longitudinell, radiell, 0)

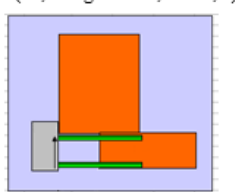

Config 2.15

(PE, Transversell, tang, 45)

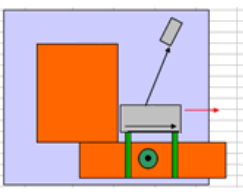

Config 2.6

(PE, Longitudinell, radiell, 0)

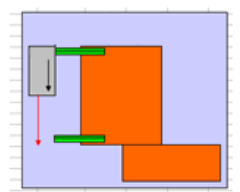

Figure 3.4: Preliminary plan of lid inspections - Ten different ultrasonic inspections originally planned for the complete inspection of the lid volume 
3. PROBLEM STATEMENT 


\section{4 \\ Study I}

As shown in section 2.5, a severity of a flaw for structural integrity is proportional to the flaw size in most of the applications. That is the reason why the POD curve is commonly derived and plotted against the size of the flaw. However, when the flaw severity for the structural integrity is determined by several parameters, the POD expressed only as function of the flaw size can not give accurate estimation of the NDT system adequacy for the inspection task.

Also, some other parameters can have as large influence on the POD as the flaw size has. The POD will not be accurate if there are other factors that influence the POD beside the flaw size.

In both cases it is important to express the POD as function of the appropriate influencing parameters.

\subsection{Influence of flaw length and flaw depth extension on the POD}

\subsubsection{Materials and methods}

Multi-parameter POD model. A first step in the analysis is to identify the most influencing parameters on the POD. This can be compared to the concept of the European network for inspection and qualification (ENIQ) of non-destructive testing [95]. It is recognized that there are many parameters that can influence the outcome of an inspection. These are defined as influential parameters. From those parameters, the ones whose change in value could affect the inspection in a way that it no longer meets 


\section{STUDY I}

defined objectives, are defined as essential parameters [96, 97]. These parameters will be application specific and depend on the inspection method and technique applied, on the apparatus used, on the material of the inspection object and on the type of the defect. The identification of the parameters will sometimes be straightforward, based on the practical experience, but sometimes additional inspections with variation of the suspected influencing parameter might be necessary. Also, the parameter size range to be investigated needs to be determined [98].

In the next step, a theoretical physical model to calculate the system response depending on the influencing parameters defined in the first step is required. Response of the system can be written as:

$$
a_{M P}=f\left(a_{1}, a_{2}, \ldots a_{n}\right)
$$

where $a_{M P}$ is the signal response amplitude that is calculated in simulation and $a_{1}, a_{2}, \ldots a_{n}$ are the influencing parameters. Since the response is a function of multiple parameters, it is referred to as a multi-parameter $a$ and denoted $a_{M P}$. Many computer simulation software tools of the most NDT inspection methods are available on the market today. Depending on the speed of calculation, which depends on the number of influencing parameters, their size range and complexity of the underlying physical model, the response can be either calculated in small increments in the defined parameter ranges or in a larger increments and a curve can then be fitted to the calculated points.

In the next step, a series of flaws with varying parameters needs to be manufactured in the test object. The number of flaws that is needed will depend on the number of parameters. The parameters should vary in the same range of concern as when calculating the response by the simulation. The inspection of the test object needs to be performed and the response recorded. It is worth noting here that the influence of several parameters on the POD could have been established by performing series of experiments on the flaws with varying influencing parameters in all combination of sizes [99]. However, the sample size is dramatically increasing with the number of influencing parameters. This number can be somewhat reduced by applying design of experiment principles, but the introduction of the model brings big advantage of the profound understanding of the underlying physical principles with even smaller sample size. 
In the next step, the signal response is calculated by the simulation with the known values of influencing parameters for each measured response of the flaw. For example, for the flaw of a certain size, orientation and depth (where size, orientation and depth are chosen influencing parameters), the response is calculated for the respective size, depth and orientation. The values are plotted in a simple scatter diagram, so that the $\mathrm{x}$-values are calculated responses, whereas the $\mathrm{y}$-values are measured responses. This diagram is very similar to the conventional $\hat{a}-a$ diagram shown in figure 2.6 .

Next, a POD calculation is performed. The calculation is basically the same as in a conventional signal response model described in section 2.5. First, a linear regression of data is performed

$$
\hat{a}=B_{0}+B_{1} a_{M P}+\delta_{M P}
$$

where $\hat{a}$ is a measured response amplitude, $a_{M P}$ the amplitude calculated with simulation and $\delta_{M P}$ is the variance of the measurement. $B_{0}$ and $B_{1}$ are the slope and intercept of the linear regression fit and can be estimated by the maximum likelihood method. If there would be no measurement uncertainties and the physical model of the inspection would be perfect, all points in the diagram would lie on a straight, $45^{\circ}$ inclined line. However, real measurements will have a variance which will result in a scatter of the points in the vertical direction. Inaccuracy of the model or not including all influencing parameters in the model, will cause a shift of points in the horizontal direction. From the vertical scatter of the points, the parameters of the underlying distribution are determined. For most applications, the normal distribution with a zero mean can be assumed. The spread of the distribution $\sigma$ is determined from the scatter of the y-values. Setting the decision threshold, the POD curve as a function of the multi-parameter $a_{M P}$ is calculated as:

$$
P O D\left(a_{M P}\right)=1-\Phi\left[\frac{\hat{a}_{d e c}-\left(B_{0}+B_{1} a_{M P}\right)}{\sigma_{\delta_{M P}}}\right]
$$

where $\Phi$ is the standard normal distribution function. Note that, unlike in the conventional analysis, there are no logarithms in the equation, because $\hat{a}$ and $a_{M P}$ are correlated in a linear scale. The curve $\operatorname{POD}\left(a_{M P}\right)$ is not easy to interpret, because it shows dependence on several combined parameters. However, since the dependence of the response on different influencing parameters is calculated by simulation, it is 


\section{STUDY I}

possible to calculate and express the POD curve as a function of one parameter at the time.

This is done in the final step, where the POD is calculated and expressed as a function of individual influencing parameters. Individual $\operatorname{POD}\left(a_{1}\right), \operatorname{POD}\left(a_{2}\right), \ldots \operatorname{POD}\left(a_{n}\right)$ curves will be similar to the conventional $\operatorname{POD}(a)$ curve and can be used to check the capability of the NDT system with respect to exactly that parameter to which the curve is expressed as a function. For example, for each parameter the $a_{90 / 95}$ point can be determined and the adequacy of the NDT system regarding that parameter can be checked.

\subsubsection{Experimental set-up}

Ultrasonic inspection of the cast iron component with a TRL probe. The multi-parameter POD model is tested on the ultrasonic inspection data from the inspection of the canister cast iron insert described in section 1.1.4. As a NDT technique for inspection of the near to surface zone, ultrasonic inspection with a transmit-receive longitudinal probe is used. The TRL probe is described in section 1.1.3. To perform reliability analysis, series of artificial semi-elliptical flaws were manufactured in the test object. The length and depth of the flaw were selected as essential influencing parameters, since from the fracture mechanics analysis severity of the flaw for structural integrity was set by those two parameters. After the inspection, the inspection data was evaluated and maximum response amplitudes were recorded for each flaw. Subsequently both the conventional and the multi-parameter POD analysis were performed and finally compared.

TRL probe. The close to surface region of the canister was inspected with four $2 \mathrm{MHz}$ TRL transducers. All transducers generate a sound field with a $70^{\circ}$ angle of incidence. The installation with the transducers positioned on the test specimen is shown in figure 4.1. First transducer is directed in the circumferential direction in the direction of the rotation of the insert, the second to the left along the length of the insert, the third to the right along the length of the insert and the fourth in the circumferential direction, opposite to the direction of the rotation. The inspection spans over the whole length of the insert and includes the depth down to $40 \mathrm{~mm}$. The aim of the inspection is to detect crack-like flaws within the inspection range. 


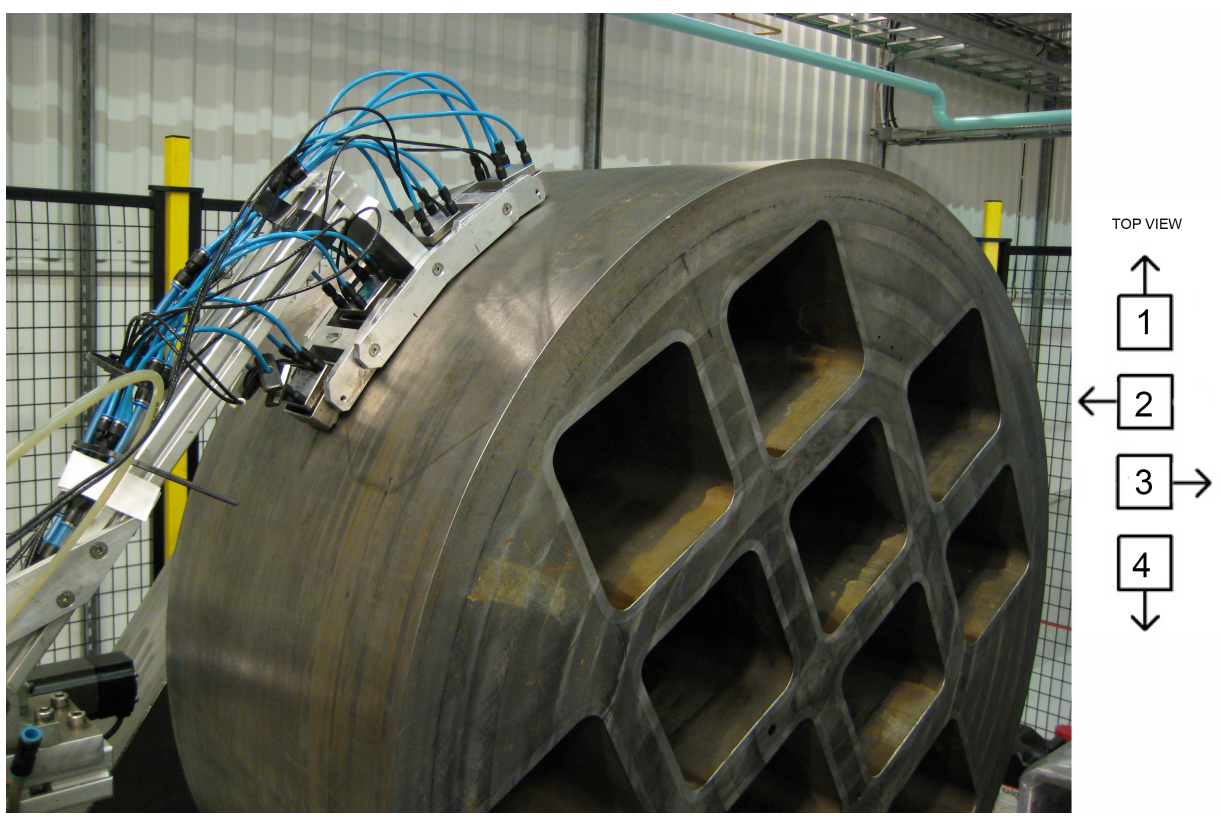

Figure 4.1: Installation with TRL probes - View of the probe installation and the insert specimen. Schematic on the right shows direction in which four TRL transducers emit the sound waves

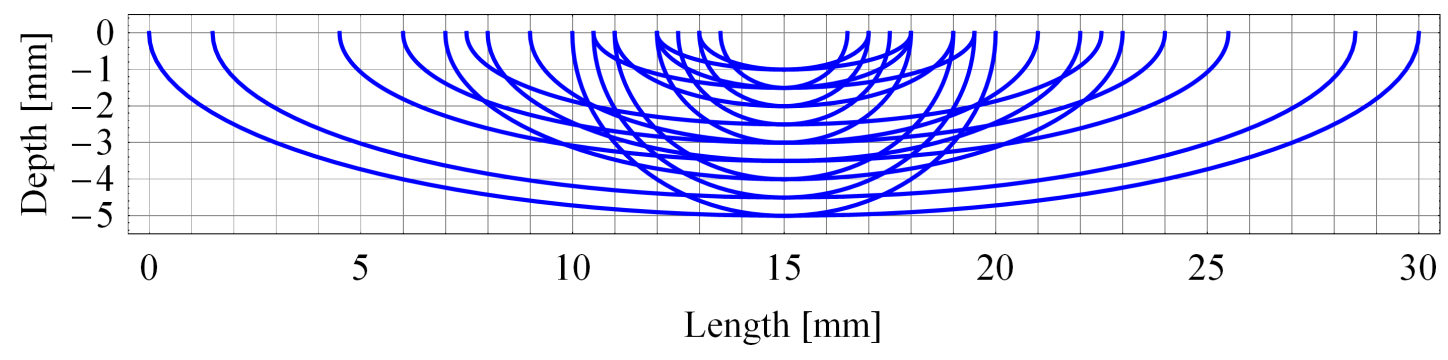

Figure 4.2: Flaws geometry - Set of semi-elliptical flaws manufactured in the test object for reliability estimation of the ultrasonic inspection system with a TRL probe

Flaws. For the analysis, 20 electric discharge machined (EDM)surface, semi-elliptical, crack-like flaws (notches) were manufactured in the test specimen. The range was defined from 1 to $5 \mathrm{~mm}$ in depth extension and from 1 to $30 \mathrm{~mm}$ in length. Flaw geometries are shown in Figure 4.2. A test object is a specimen that can be easily inset into the original canister geometry, so that the whole inspection set-up for the inspection of the test object is exactly the same as for the inspection of the real canister. A technical drawing of the test specimen with flaws is shown in appendix, figures B.1 and B.2. 


\section{STUDY I}

Simulation. For the numerical simulation of the inspection process, the BAM simulation software was used, as described in section 2.6. The calculation was performed in two steps. First, the sound field generated by the specific TRL probe in the material was calculated. Figure 4.3 a) shows the vertical cross section of the sound field, as calculated by the simulation software. In figures $4.3 \mathrm{~b}$ ) and $c$ ), sound field in the horizontal plane inclined $85^{\circ}$ and $70^{\circ}$ respectively, is shown. In the second step response signal amplitudes for flaws were calculated. The response was calculated in the same size range of depth extension and length of the flaw as for the manufactured flaws.

\subsubsection{Results and Discussion}

The reliability analysis is first performed in a conventional way. Measured response signal amplitudes from the ultrasonic inspection are plotted against the flaw size (notch area) in a log-log diagram as shown in figure 4.4, showing the signal-to-noise ratio (SNR) versus the flaw size. The solid line shows a linear regression fit. The decision threshold is set to $\mathrm{SNR}=3$, indicated with the horizontal red line. Amplitudes that are 3 times larger than the noise are seen as a signal. This is a conservative assumption, since signals with even smaller SNR can be easily identified, as shown in section 2.3 .

The POD and lower 95\% confidence band are then calculated and are shown in figure 4.5. The solid line represents the POD curve and the dotted line the lower $95 \%$ confidence band. The $a_{90 / 95}$ point is indicated with the intersection of two green lines and corresponds to the flaw with the area of $15.9 \mathrm{~mm}^{2}$. If the $a_{90 / 95}$ is used as a measure of system capability of detecting flaws, the result of the reliability analysis would be that if the flaw that needs to be detected has an area of $15.9 \mathrm{~mm}^{2}$ or larger, the NDT system can reliably be used for the inspection of the component.

In the next step, the multi-parameter analysis is performed. Figure 4.6 shows the amplitude of the response signal plotted as a function of the flaw depth and length, as calculated by the simulation. It can be seen that the amplitude is increasing with the increasing length and depth extension of the flaw. At some point, the reflected amplitude reaches a constant value or becomes saturated. The size of the flaw becomes larger than the local sound field cross-section, so that an increase in the flaw size does not influence the reflected amplitude any more.

When the measured response amplitudes are plotted against the amplitudes calculated by the simulation, the $\hat{a}$ versus $a_{M P}$ diagram shown in figure 4.7 is created. The 


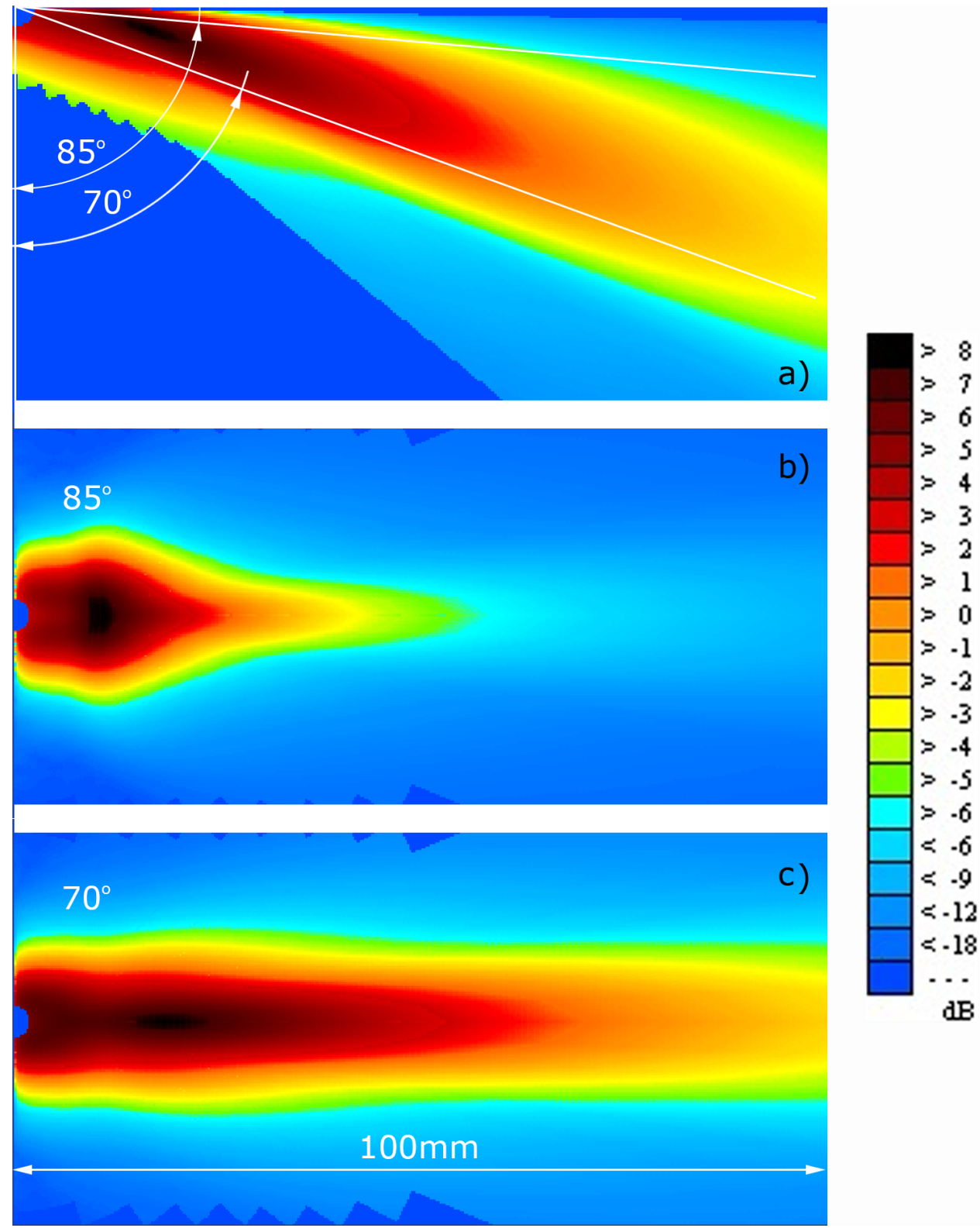

Figure 4.3: Simulation of the TRL sound field - a) side view, b) top view of the plane rotated $85^{\circ}$ and top view of the plane rotated $70^{\circ}-\mathrm{c}$ ) 


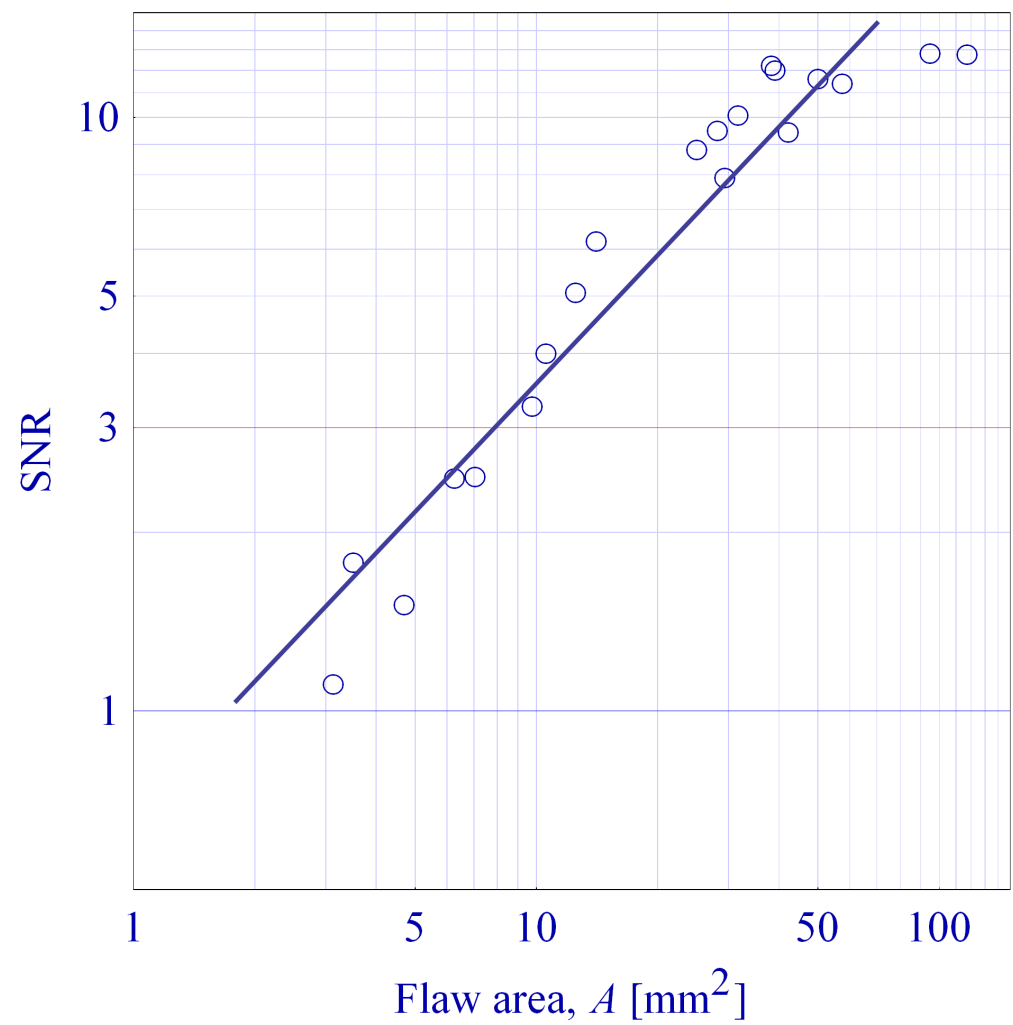

Figure 4.4: Conventional $\hat{a}$ versus $a$ diagram - Measured amplitude as a function of a flaw area with regression line in logarithmic scale 


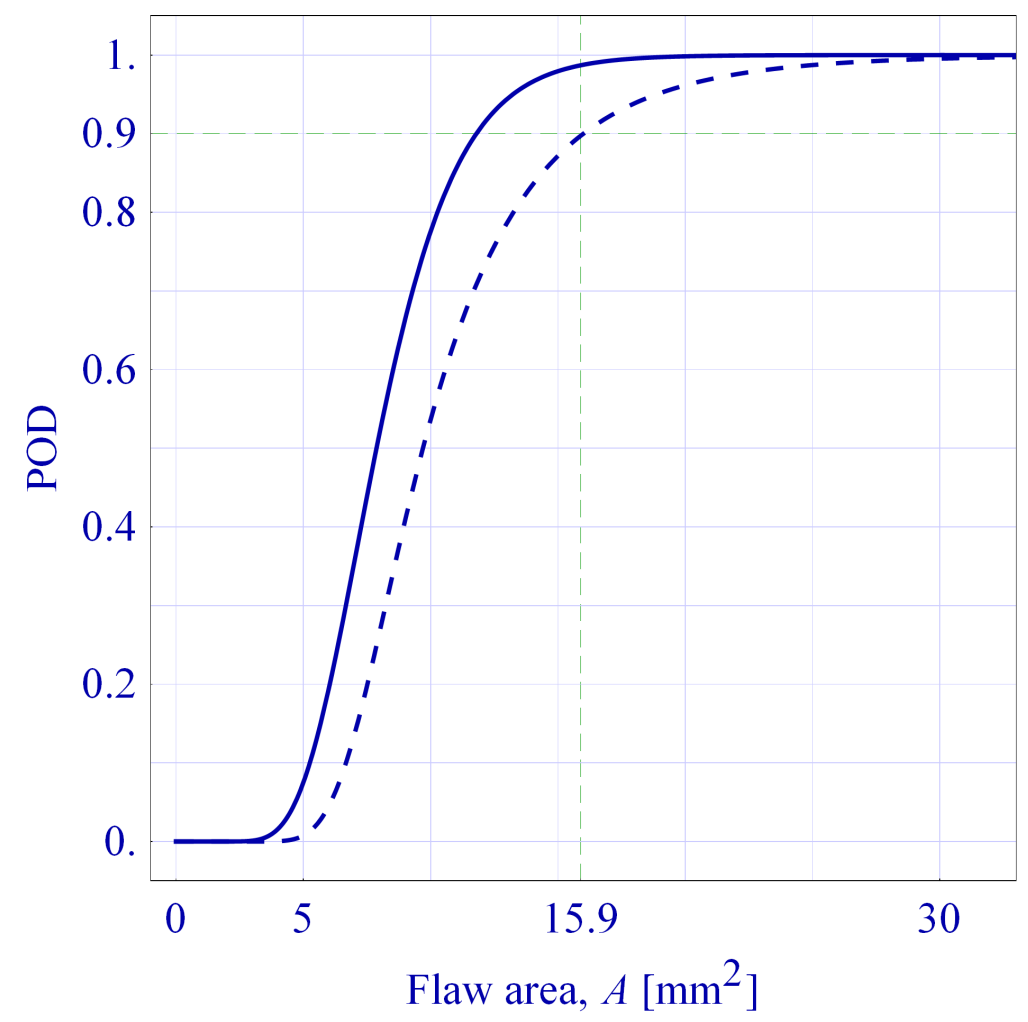

Figure 4.5: $P O D(a)$ curve - POD as a function of a flaw area, as calculated by the conventional signal response analysis

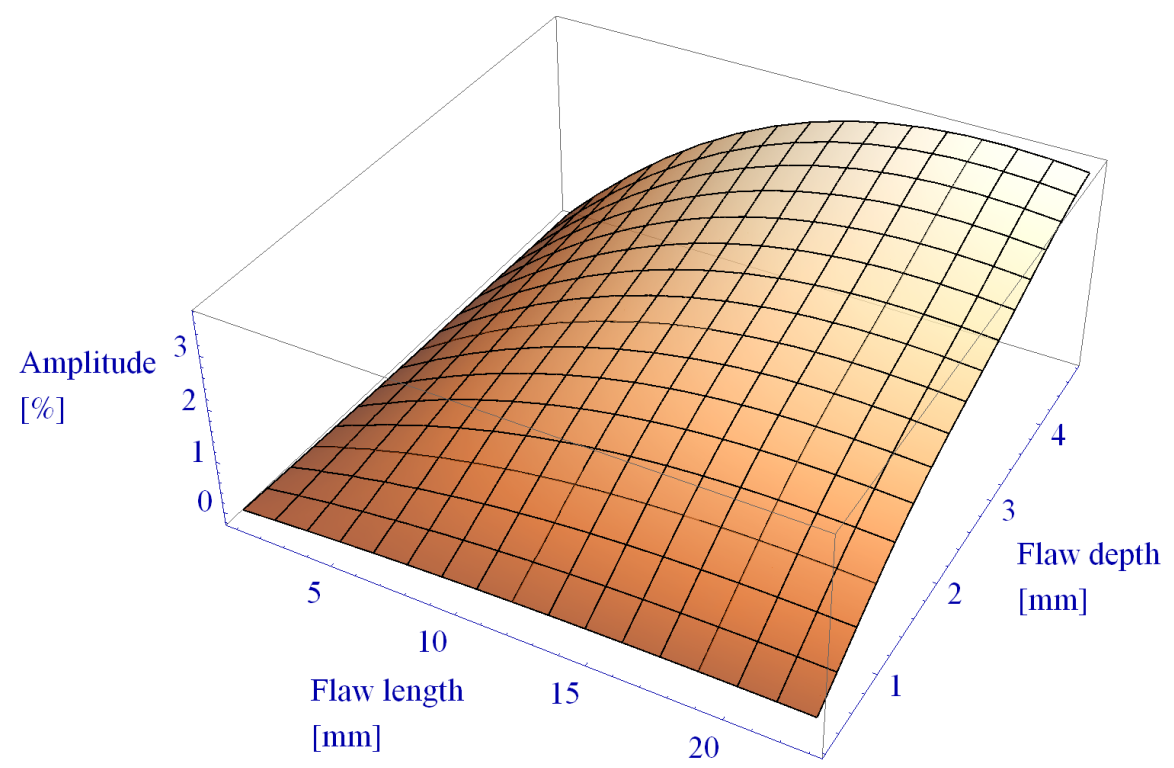

Figure 4.6: Simulated signal response - Signal response of a flaw as a function of flaw length and depth, as predicted by the simulation 


\section{STUDY I}

dotted line represents a linear regression fit of the data. The regression line is close to the ideal, $45^{\circ}$ line, which shows good agreement of the simulation with the experiment. Small scattering signifies a small variance of the measurement.

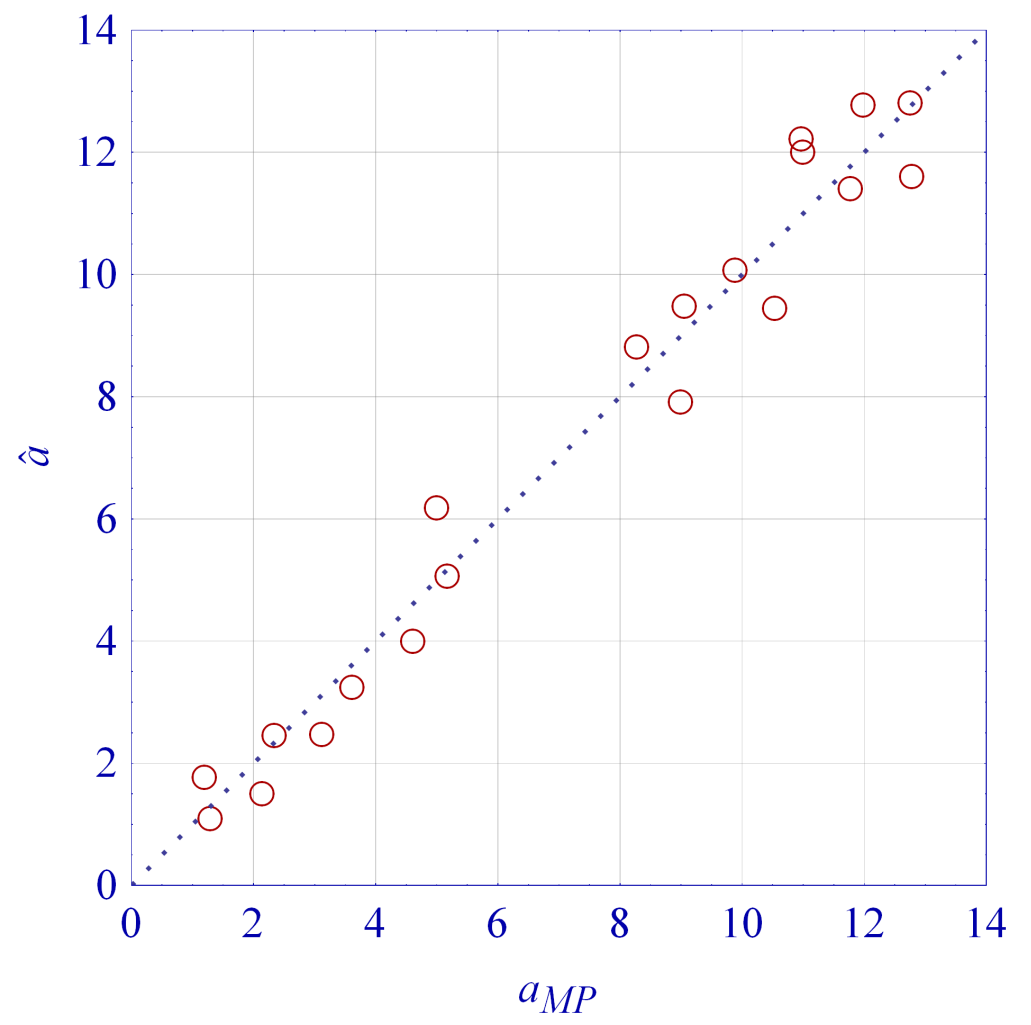

Figure 4.7: Multi-parameter $\hat{a}-a_{M P}$ diagram - Calculated with the multi-parameter POD model

The decision threshold was set at $\mathrm{SNR}=3$, same as in the conventional POD calculation. Assuming normally distributed variance, the POD is calculated and shown as surface in figure 4.8. The POD surface shows that for the increasing depth extension and length of the flaw, the POD is increasing from 0 to 1 . Two regions can be identified: a region where the POD is increasing from 0 to 1 and a region where the POD is practically 1 . This kind of representation of the POD shows qualitative influence of the multiple parameters on the POD, but quantitative readings can be ambiguous. Additionally, if more than two parameters are included in the analysis, the POD will be multi-dimensional, which will make a graphical representation impossible. By keeping all except one parameter constant, the POD can be presented in a conventional way, 
as described below

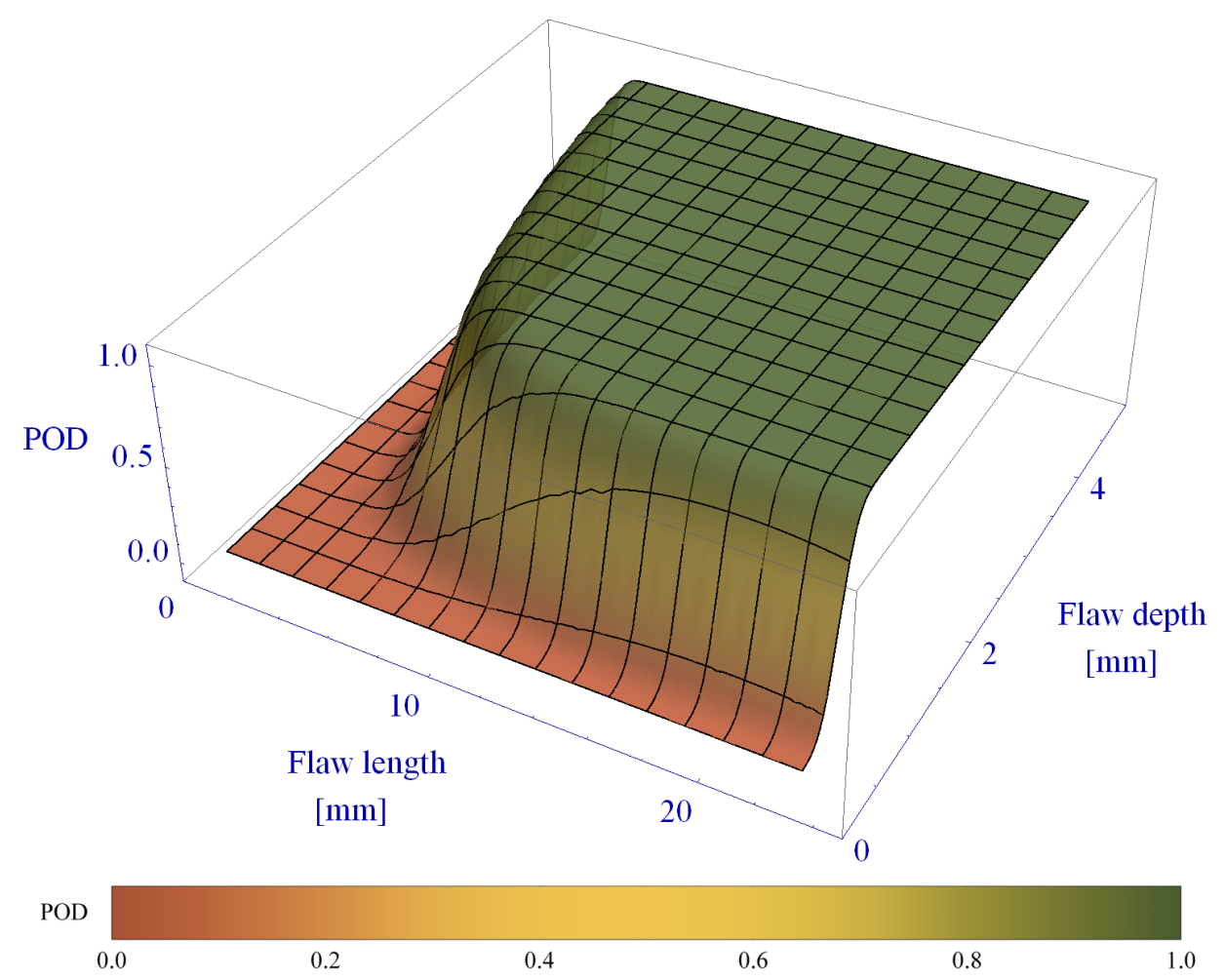

Figure 4.8: $P O D(d, l)$ - POD as a function of a flaw depth and length

Figure 4.9 a) shows the POD as a function of the flaw depth calculated for the three flaw lengths: 5, 10 and $15 \mathrm{~mm}$. For clarity, only the lower $95 \%$ confidence bands are plotted. The diagram shows that if the critical flaw is $5 \mathrm{~mm}$ long, the NDT system can only be safely used for the inspection if the flaw is at least $3.5 \mathrm{~mm}$ deep (the $a_{90 / 95}$ point is at about $3.5 \mathrm{~mm}$ ). If, on the other hand, the critical flaw that needs to be detected is $15 \mathrm{~mm}$ long, it will be detected with sufficiently high probability if it is only $1 \mathrm{~mm}$ deep or deeper.

Figure 4.9 b) shows the POD curves, derived from the same POD surface shown in figure 4.8 , but as a function of the flaw length, calculated for three different flaw depths. Again, for clarity reasons, only the lower 95\% confidence bands are plotted. It can be seen from the diagram that the flaw $1.5 \mathrm{~mm}$ deep, has to be at least $8 \mathrm{~mm}$ long to be reliably detected. If the flaw is $5 \mathrm{~mm}$ deep, required minimal length of the flaw for reliable detection is about $4 \mathrm{~mm}$. 

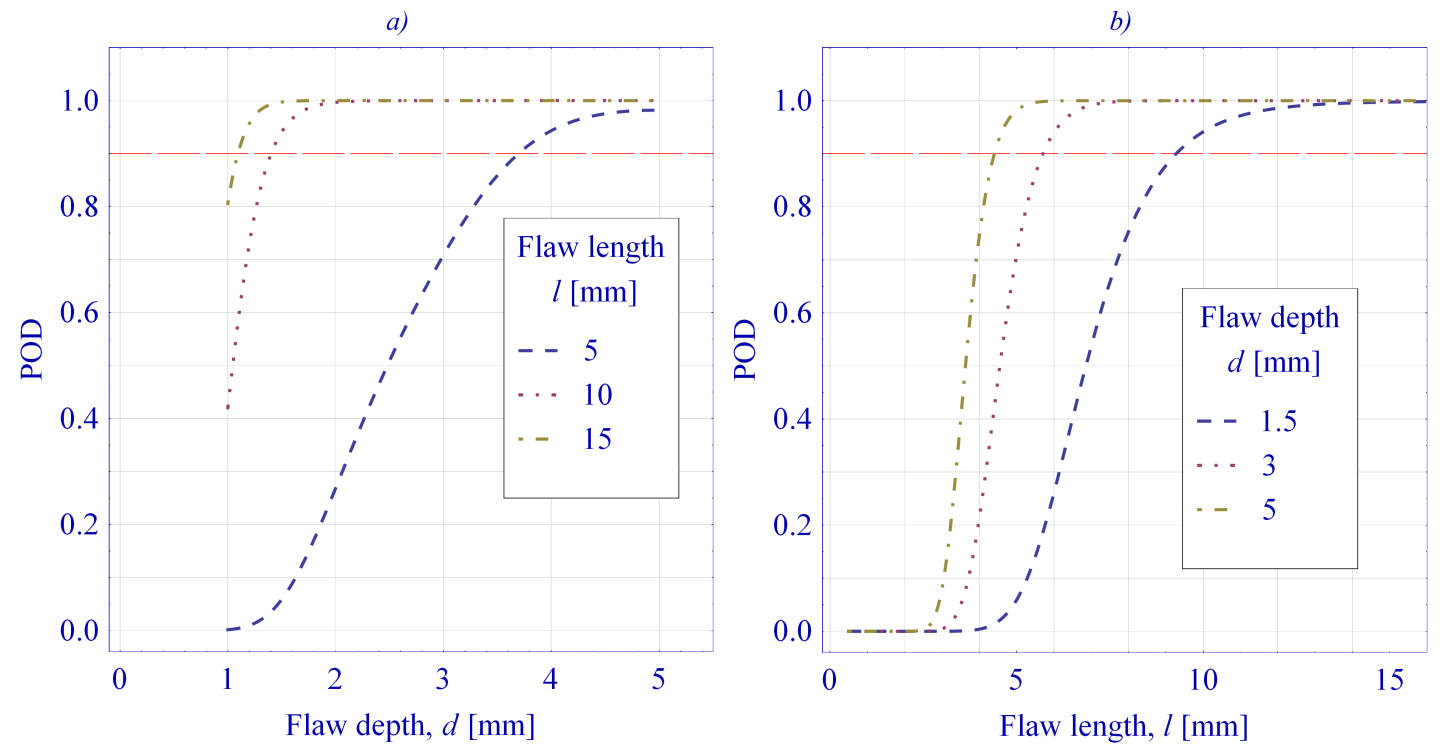

Figure 4.9: $\operatorname{POD}(d, l=$ const $)$ and $P O D(d=$ const,$l)$ - Lower $95 \%$ confidence band as a function of the flaw depth for different flaw lengths a) and as a function of the flaw length for different flaw depths $b$ )

To make a direct comparison with the POD curve calculated with the conventional signal response model shown in figure 4.5 , the diagram shown in figure 4.10 is created. Once again, for clarity reasons, only lower 95\% confidence bands are plotted in the diagram. The $95 \%$ confidence bands are shown as a function of the flaw area $A$, calculated for three different flaw depths (dashed lines) with the multi-parameter model and calculated with the conventional signal response model (solid line). At the first look it can be misleading that the curves for the shallower flaws lie left from the curves of deeper flaws, which in the conventional POD diagram would mean a higher POD. The right way to interpret the diagram will be explained on the example. The flaw with the area of about $12.5 \mathrm{~mm}^{2}$ which is $5 \mathrm{~mm}$ deep, will have the POD of about only $20 \%$. The flaw of the same area but only $3 \mathrm{~mm}$ deep will have the POD of around $90 \%$. Since both flaws have the same area, that means that the shallower flaw is longer than the deeper one. It follows that not only the area of the flaw, but its aspect ratio will influence the POD. A reason for this is a point-asymmetric shape of the sound field created by the TRL probe, as illustrated in figure 4.3. This phenomenon is included in the multi-parameter model and neglected by the conventional model.

There is a region of parameter sizes where flaws which are longer but shallower will 


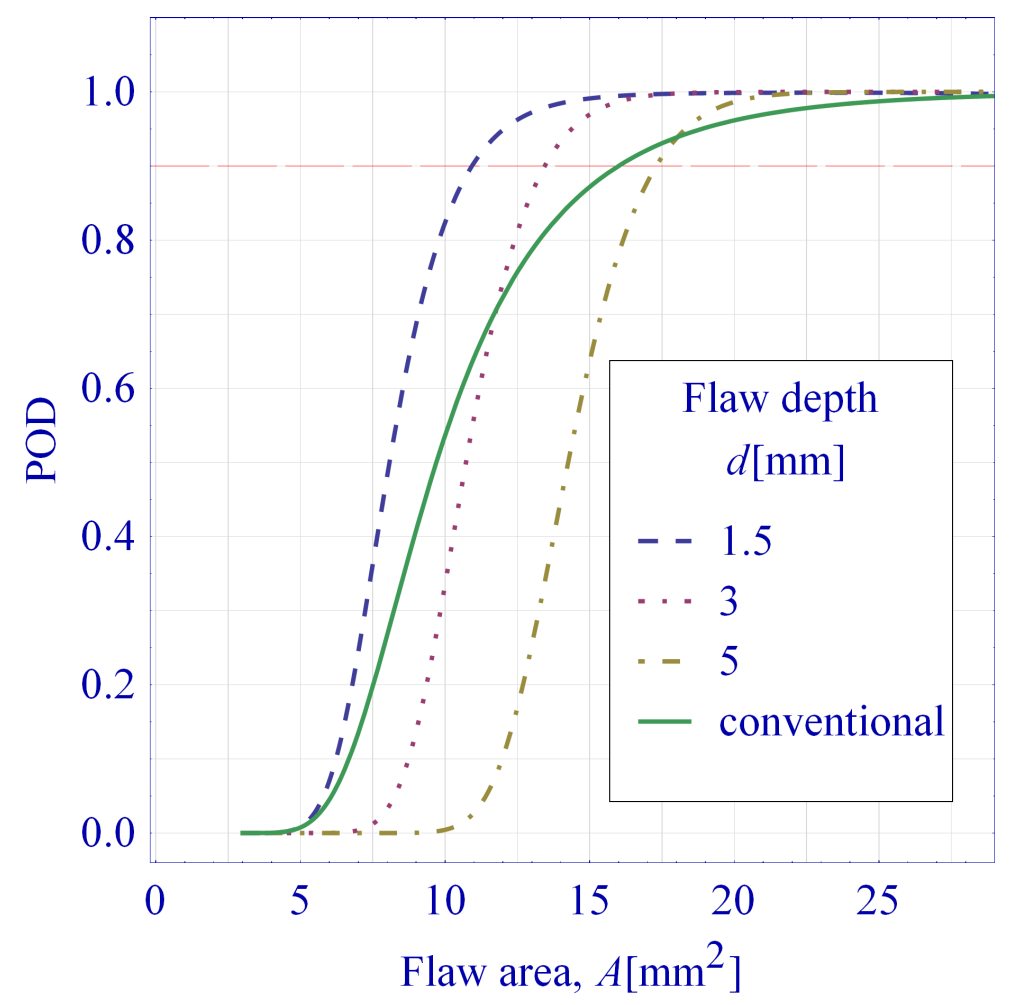

Figure 4.10: Comparison of the conventional and multi-parameter POD - Lower $95 \%$ confidence bands as a function of the flaw area, as calculated by the multi-parameter analysis (dashed lines) and by the conventional signal response analysis (solid line) 


\section{STUDY I}

have higher POD than the flaws that are deeper but shorter, having the same area. This can be seen in figure 3.1 although both flaws have the same area, the left one will have a higher POD than the right one. This information can not be extracted from the POD curve calculated by the conventional signal response model. If, for example, the flaw with $10 \mathrm{~mm}^{2}$ area would be a critical one, the method would overestimate the POD for shallow flaws (gives 0.9 for $1.5 \mathrm{~mm}$ deep flaw) and underestimate the POD of deep flaws (almost zero for $5 \mathrm{~mm}$ deep flaw) compared to the POD curves from the multi-parameter model.

\subsection{Influence of ultrasonic attenuation on the POD}

As mentioned in section 2.5, when inspecting thin components or components with small attenuation, the influence of attenuation on the POD of the ultrasonic inspection system is negligible. In this section, ultrasonic inspection of thick copper components with high and variable attenuation will be shown. In this case, the depth of the flaw as well as the attenuation coefficient will be taken as parameters into the multi-parameter POD model calculation.

\subsubsection{Materials and methods}

Ultrasonic attenuation as an influencing parameter. When the attenuation of the ultrasonic wave in the material is influencing the POD considerably, the attenuation coefficient should be selected as a parameter for the multi-parameter POD model. Some other parameters like the flaw size and depth will be selected as parameters as well. In the multi-parameter model, for all influencing parameters the size range of interest should be determined and flaws with parameters varying in those ranges manufactured. The same is true for the attenuation coefficient. The attenuation coefficient echoes the material structure. In order to carry out a valid POD analysis, test specimens with the grain size, shape and distribution that varies in the same range as expected in the production components, should be available for the POD study. Since it is technically difficult to produce specimens with controlled material structure, the specimens should be selected randomly from the production parts, so that the attenuation in the test specimens matches the attenuation in real components as much as possible. Flaws with 
parameters varying in the ranges of interest of the parameter are then manufactured in the specimens. Inspection is performed and response signal amplitudes are recorded.

The POD model is based on the comparison of the response signal amplitudes measured with the NDT system (which can be seen as an apparent flaw size) and the true size of the flaw. The true size is in fact also a measurement of the flaw size but with a measurement system of much higher precision. For the multi-parameter model, true values of all influencing parameters are also needed as an input for the simulation. Geometrical parameters like length of the flaw and its depth can be measured precisely whereas the attenuation coefficient $\alpha$ not. One option to determine $\alpha$ is from the relationship between $\alpha$ and grain size that can be modelled by the following relation [100]:

$$
\text { for } \lambda \gg 2 \pi \bar{D}, \alpha \propto \bar{D}^{3} f^{4}
$$

where $\bar{D}$ is the average grain diameter, $\lambda$ is the wavelength and $f$ is a frequency. This method is not very practical for the measurement and also the grain shape and orientation distributions of grains that also influence the attenuation [101, 102], are not taken into account. As a reliable method for determination of $\alpha$ with ultrasound, an immersion method is recommended [103]. This method gives accurate results for transducers that do not have large diffraction of the beam. If diffraction of the beam is large, the amplitudes need to be corrected for the diffraction. There are also other methods proposed for the attenuation measurement, e.g. using through transmission technique [104] or buffer rod method [105].

In this example the attenuation coefficient is estimated from the measurement of multiple back-wall echoes amplitudes from a specimen with plan-parallel surfaces. Measured repeated back-wall echo amplitudes contain influences of the three mechanisms described in section 1.1.3. To isolate the influence of the attenuation on the amplitude, the influence of change of amplitude on the material boundaries and the diffraction of the beam are subtracted from the measured amplitudes. The change of amplitude on material boundaries is calculated from the transmission and reflection coefficient of the used materials, and the change due to the diffraction of the beam is calculated with the simulation software. The observed drop of the repeated back-wall amplitudes is now caused by the attenuation in material only, which is assumed to be exponential, as expressed in equation 1.4. The attenuation coefficient $\alpha$ is determined from the leastsquare fit of the exponential function to the back-wall echo amplitudes. The back-wall 


\section{STUDY I}

echo amplitudes are measured in the near vicinity of each flaw to determine a local coefficient of attenuation $\alpha_{i}$, where $i$ is the number of the flaw.

Theoretical response amplitudes are now calculated for the flaws by varying all parameters in the range of interest. The $\hat{a}-a_{M P}$ diagram is created by plotting the measured values of the amplitudes against the values theoretically predicted by the simulation. Setting the decision threshold the POD curve as a function of multi-parameter $a_{M P}$ is calculated. Having all except one influencing parameter constant, the POD curve can be expressed as a function of that parameter. In the case of ultrasonic attenuation, the POD can be expressed as a function of the attenuation coefficient $\alpha$.

\subsubsection{Experimental set-up}

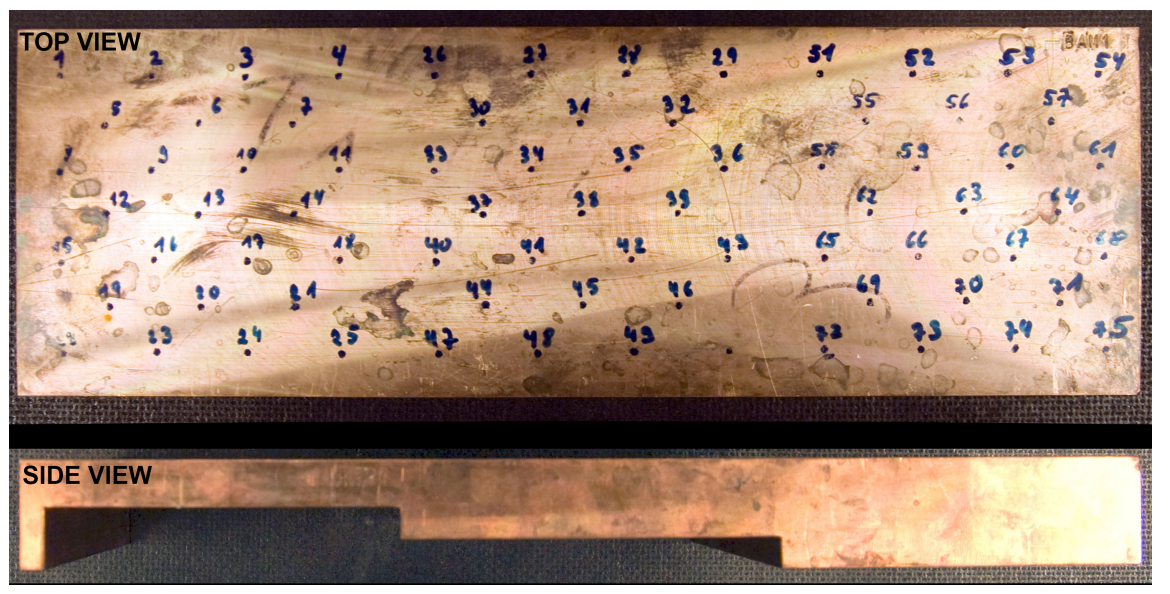

Figure 4.11: Copper specimen - Top and side view of the copper specimen

Ultrasonic inspection of the thick copper component. The application of the multi-parameter model, taking the attenuation coefficient as an influencing parameter, is demonstrated with the ultrasonic phased-array inspection data of the thick copper components. Test specimens used for the POD analysis were cut-out blocks from the copper tube manufactured in the same way that the tubes for the permanent storage will be manufactured once the repository is operational. This copper tube displayed high variation in ultrasonic attenuation. One of the specimens is shown in figure 4.11. Technical drawings of both specimens are shown in appendix, figure A.1 and figure A.2. It can be seen in the side view that specimens were cut stepwise, to have different thicknesses. This way the drilling of very long holes from the bottom side was avoided, 
but still made it possible to have flat-bottom holes in the wide range of depths. 165 flatbottom holes were manufactured in the specimens, 150 of them used in the experiment. The range of interest for diameter was 1.0 to $3.0 \mathrm{~mm}$ and for depths 6.0 to $60.0 \mathrm{~mm}$. There were three instances of each flat-bottom hole with a specified diameter and depth with random location in the specimen, ensuring a different local attenuation in material.

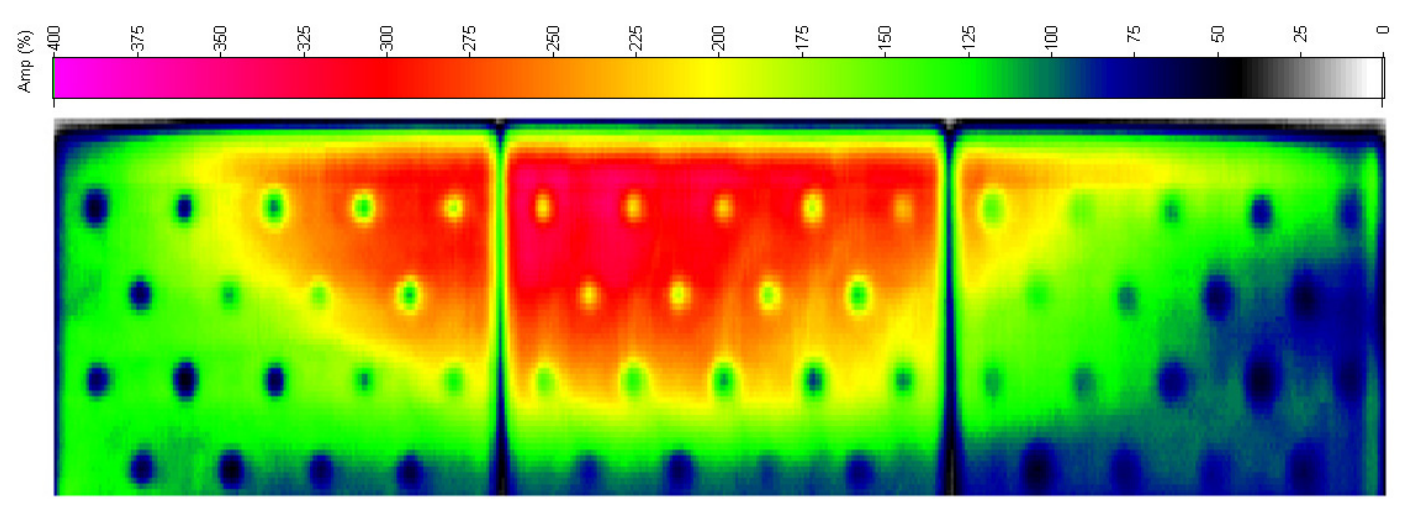

Figure 4.12: C-scan - Ultrasonic C-scan of the copper sample, showing variation in the back-wall echo amplitudes

The specimens were inspected and inspection data recorded. The C-scan from the inspection is shown in figure 4.12. The change of the back-wall amplitude across the sample also indicates a change in the attenuation of the ultrasonic wave. The smallest back-wall echo amplitude measured in the specimens was 80 and the largest was 330 on linear scale of the evaluation software. This is more than $410 \%$ difference between the strongest and weakest back-wall echo amplitude.

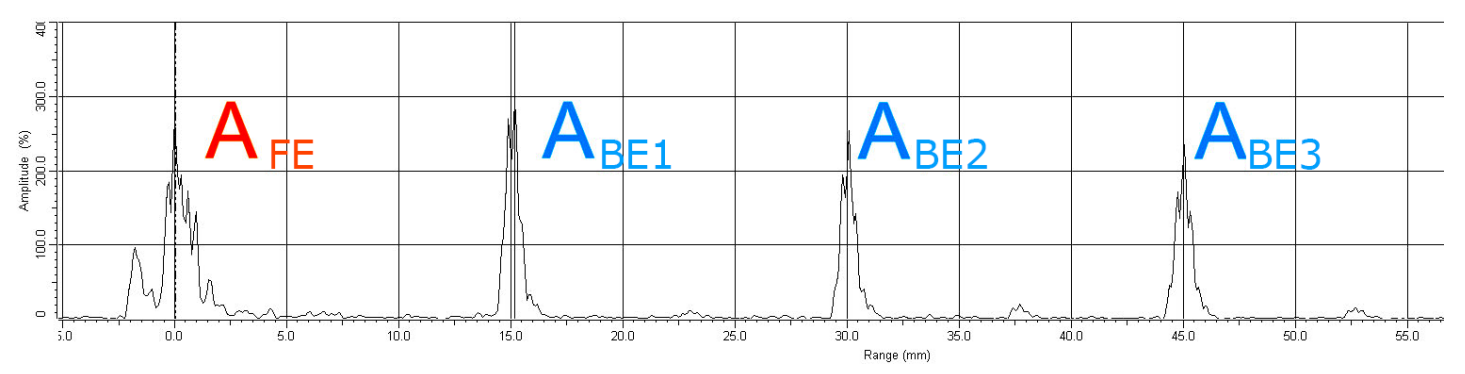

Figure 4.13: A-scan - A-scan showing front-wall and multiple back wall echoes. Echoes are designated according to figure 4.14

To determine a local coefficient of attenuation $\alpha_{i}$, three back-wall echoes were recorded in the thinnest part of the specimen. The A-scan showing multiple back-wall 


\section{STUDY I}

echoes is shown in figure 4.13. The illustration explaining the echoes origin is shown in figure 4.14. The ultrasonic wave excited by the transducer after travelling through the immersion medium, impinges on the front surface of the copper specimen with the amplitude $A_{0}$. The wave gets reflected from the front wall with the amplitude $A_{F E}$. This amplitude is related to the incidence wave amplitude as $A_{F E}=R A_{0}$, where $R$ is a reflection coefficient calculated according to equation 1.1. The wave also enters the copper specimen with the amplitude $T A_{0}$, where $T$ is a transition coefficient according to equation 1.2 . The wave will propagate through the copper according to the laws of the beam diffraction and attenuation in material, as explained in 1.1.3, until it reaches the back surface of the specimen. Again, the wave will be reflected and transmitted. The reflected wave, will travel back through the specimen, reach the front surface with the amplitude determined by the laws of the beam spread and attenuation and transmit through the surface with the amplitude $A_{B E 1}$ which will be recorded by the NDT system as a first back-wall echo. The same principle applies for the amplitudes $A_{B E 2}$ of the second and $A_{B E 3}$ of the third back-wall echo.

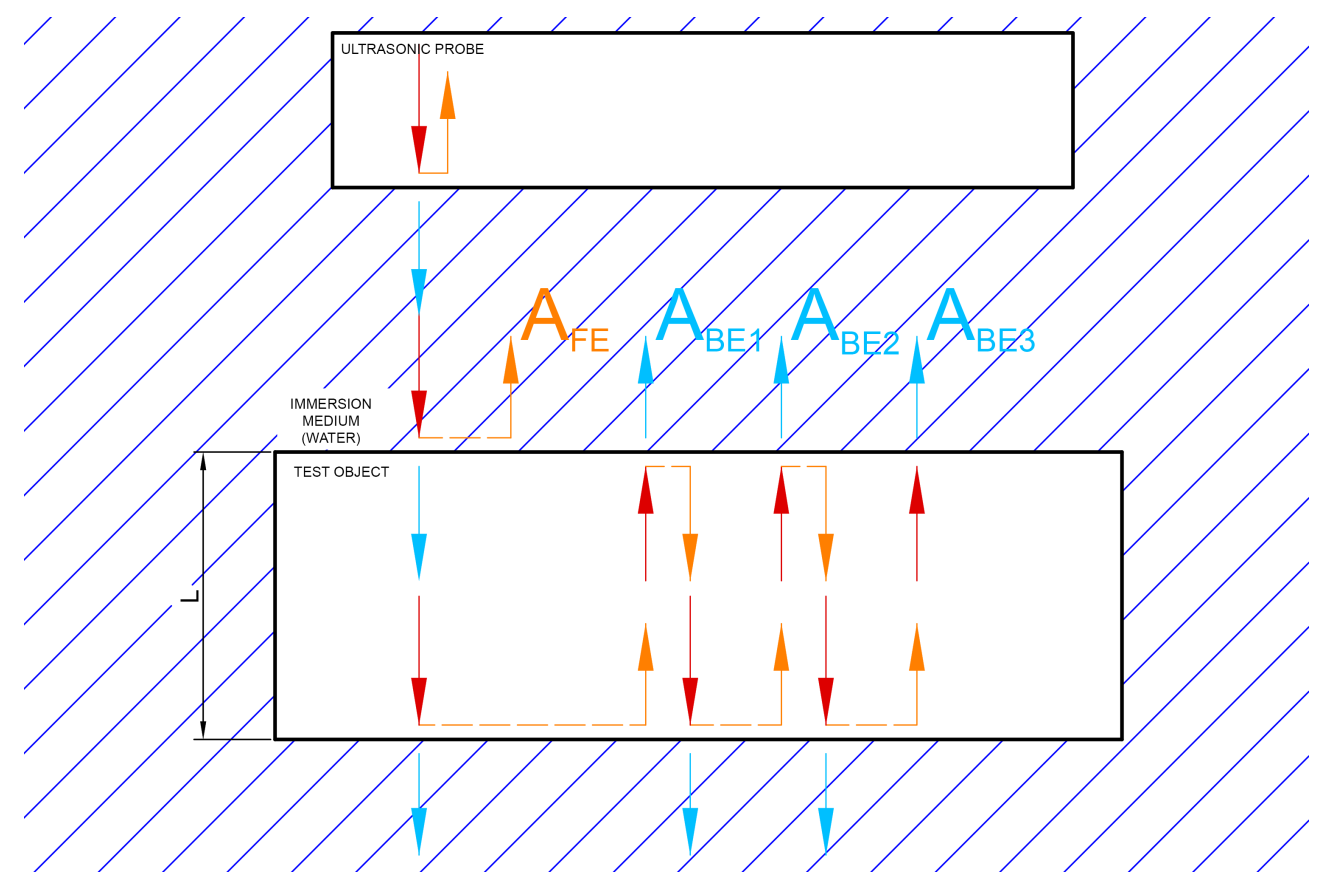

Figure 4.14: Ultrasonic wave amplitude in the specimen with plan-parallel surfaces - Schematic representation of the reflected (orange) and transmitted (blue) amplitudes of the normal incident (red) ultrasonic wave in the specimen with parallel surfaces 


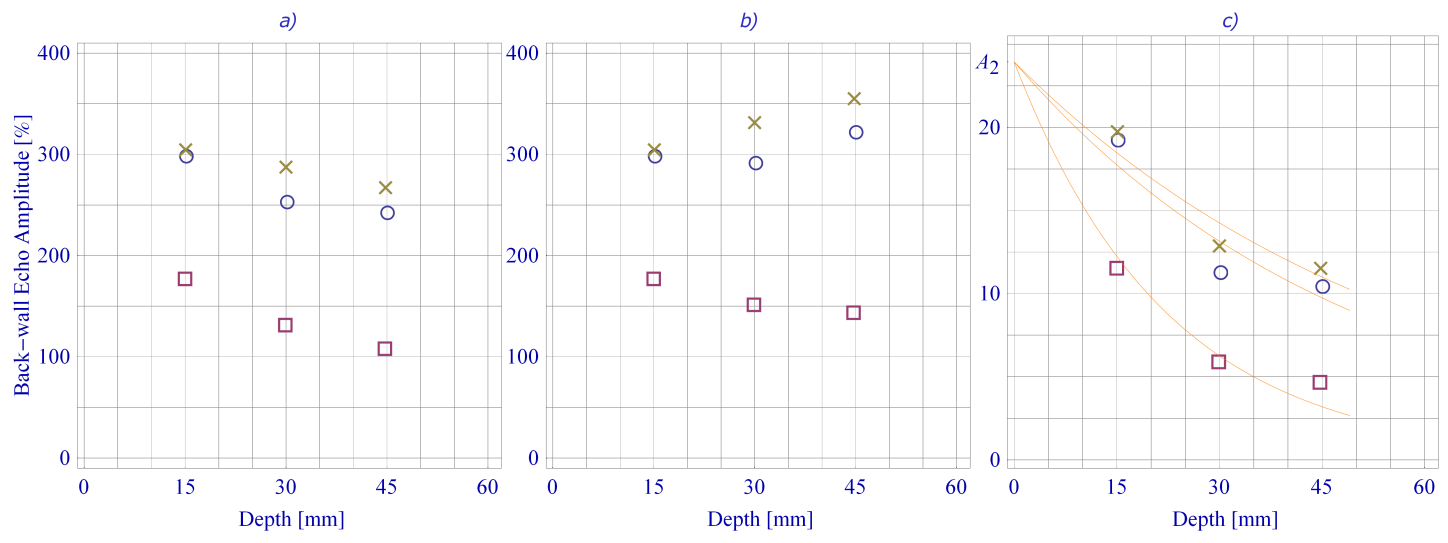

Figure 4.15: Multiple back-wall echo amplitudes - a) measured repeated backwall echoes on three different places on the test object, b) magnitudes corrected for the amplitude change on the boundaries between copper and water, c) magnitudes corrected for the beam spreading with the attenuation exponential function fit

The diagram in Figure 4.15 (a) shows amplitudes of the three repeated back-wall echoes plotted against the depth. These echoes correspond to echoes $A_{B E 1}, A_{B E 2}$ and $\left.A_{B E 3}\right)$ from figure 4.14. Echoes are measured at the three different locations on the specimen, marked with different symbols. These amplitudes reflect the change of the amplitude with the distance due to the boundary effect, beam spreading and attenuation. The only change from location to location on the specimen is the material below the transducer, so the difference between echo amplitudes must come from the material. In figure 4.15 (b) these amplitudes are corrected for the change on the specimen boundaries. Points in diagram show change of the amplitude with a distance if the wave would be propagating through a material without boundaries. In figure 4.15 (c) these amplitudes are now corrected for a beam spreading. Diagram shows change of the wave amplitude caused by the attenuation only. Assuming an exponential change, curves are fitted through the points. The front-wall echo $A_{F E}$ and local attenuation coefficient $\alpha_{i}$ are determined from the fit. Three repeated back-wall echoes were recorded only in the thin part of the specimen. In the thicker parts only two, or in the thickest part only one, back-wall echo was recorded. Assuming the front-wall echo $A_{F E}$ constant across the specimen, local coefficient of attenuation in thicker parts is calculated by fitting the exponential curve to one point.

Once the local $\alpha_{i}$ is determined, the simulation of the response amplitude for flatbottom holes varying in size and depth in the range of interest was calculated. Values 


\section{STUDY I}

measured were plotted against the theoretically predicted values, creating the $\hat{a}-$ $a_{M P}$ diagram. The decision threshold was set at $3 \mathrm{SNR}$ and the $\operatorname{POD}\left(a_{M P}\right)$ was calculated. The POD as a function of depth $\operatorname{POD}(z)$ for the flat-bottom hole with specified diameter and several $\alpha$ was calculated in the next step. Also the POD as a function of depth $\operatorname{POD}(z)$ for the $\mathrm{FBH}$ in a material with specified attenuation coefficient $\alpha$ and several diameters was calculated.

\subsubsection{Results and discussion}

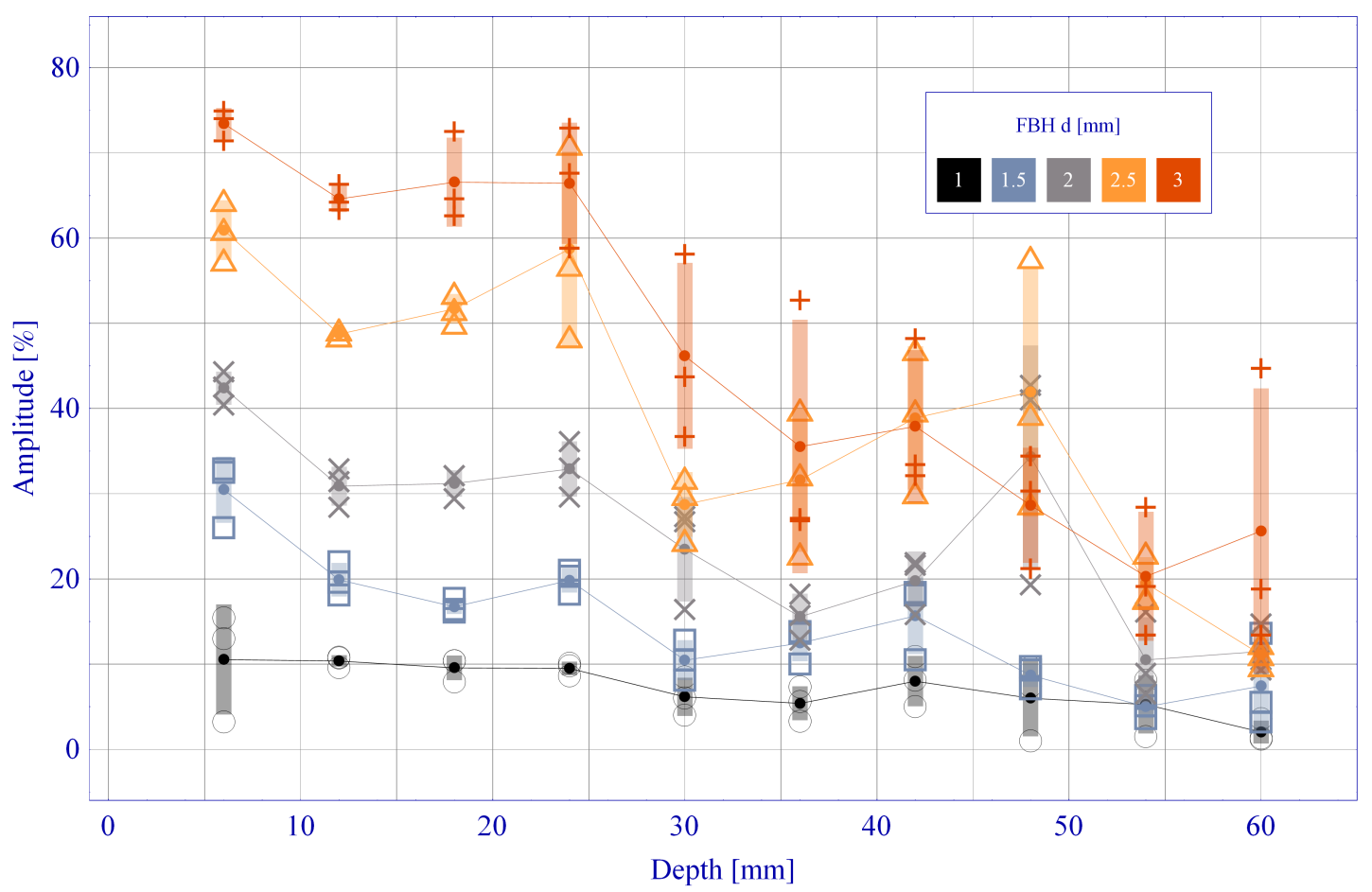

Figure 4.16: FBH response amplitudes - Measured response amplitude of the flatbottom holes

Figure 4.16 shows measured response amplitudes from 150 flat-bottom holes. Diameters are distinguished by different colors. Standard deviation and mean value of the three measurements of three flat-bottom holes with the same diameter and depth are marked with a transparent square field and a solid point, respectively. Mean values for the flat-bottom holes with the same diameter are connected with a straight line for clarity of presentation. It would be expected that lines follow the shape of the overall amplitude change from figure 1.4. However, it can be seen from the diagram that 
FBHs with a smaller diameter have higher response amplitude than those with a larger diameter in the same depth. Obviously, there is another parameter that is influencing response amplitude, beside the flaw size and depth.

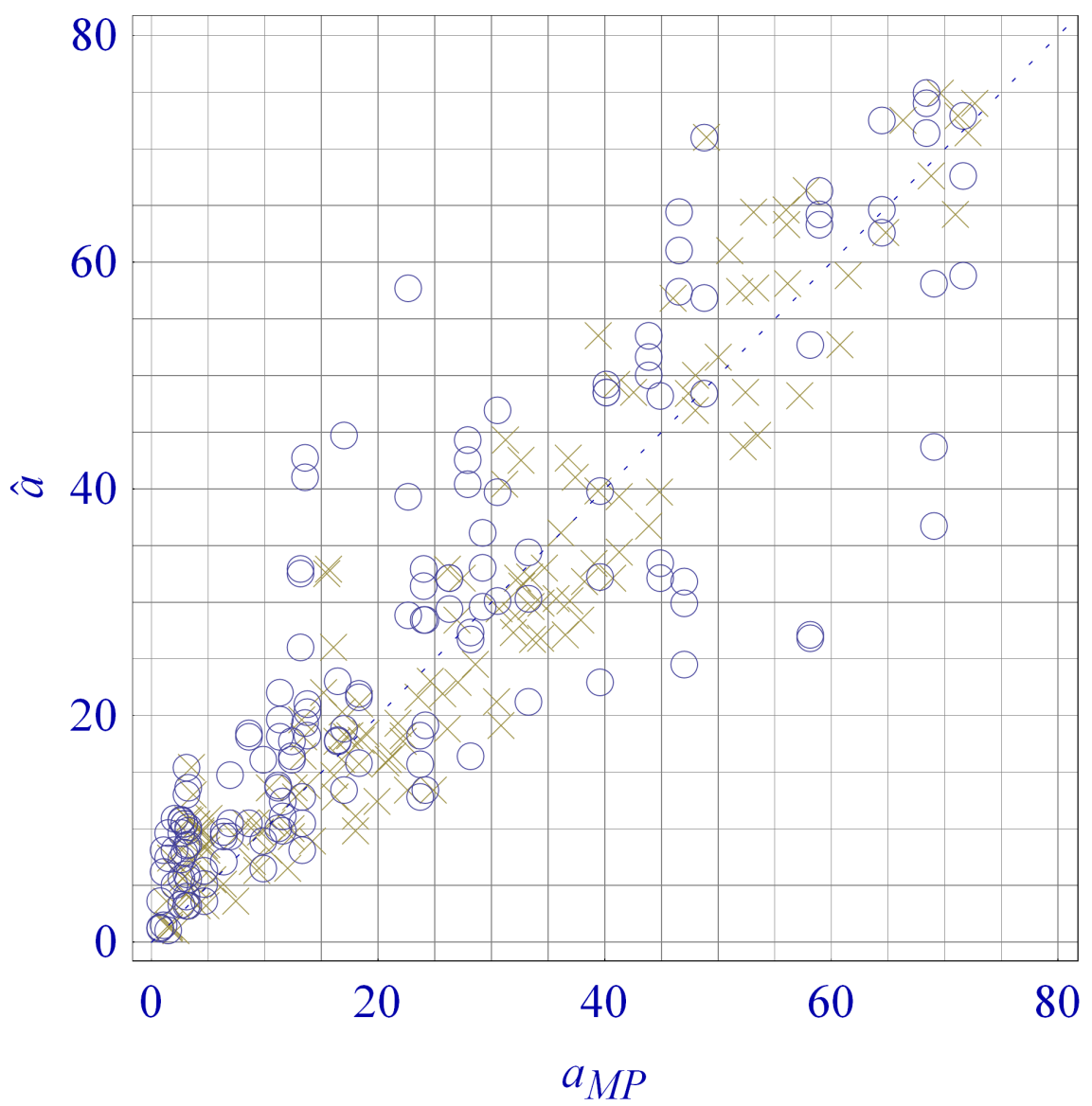

Figure 4.17: $\hat{a}-a$ diagram - as calculated by the multi-parameter analysis. Calculation with the constant attenuation coefficient (circles) and local, variable attenuation coefficient (crosses)

Plotting measured response amplitudes versus amplitudes calculated by the simulation, the $\hat{a}-a_{M P}$ diagram is created, as shown in figure 4.17. Points are plotted for two cases of simulated amplitudes. The circles represent responses calculated with one constant attenuation coefficient $\alpha$ for the whole specimen. The crosses represent responses calculated with the local attenuation coefficient $\alpha_{i}$. It can be seen that that scatter of the points around the ideal, $45 \mathrm{deg}$ line is higher for the constant $\alpha$ (root 


\section{STUDY I}

mean square $\mathrm{RMS}=10,45)$, than for the variable local $\alpha_{i}(\mathrm{RMS}=6,05)$. This indicates that the attenuation coefficient is indeed an influencing factor and that including it in the multi-parameter model will improve the estimate of the POD.

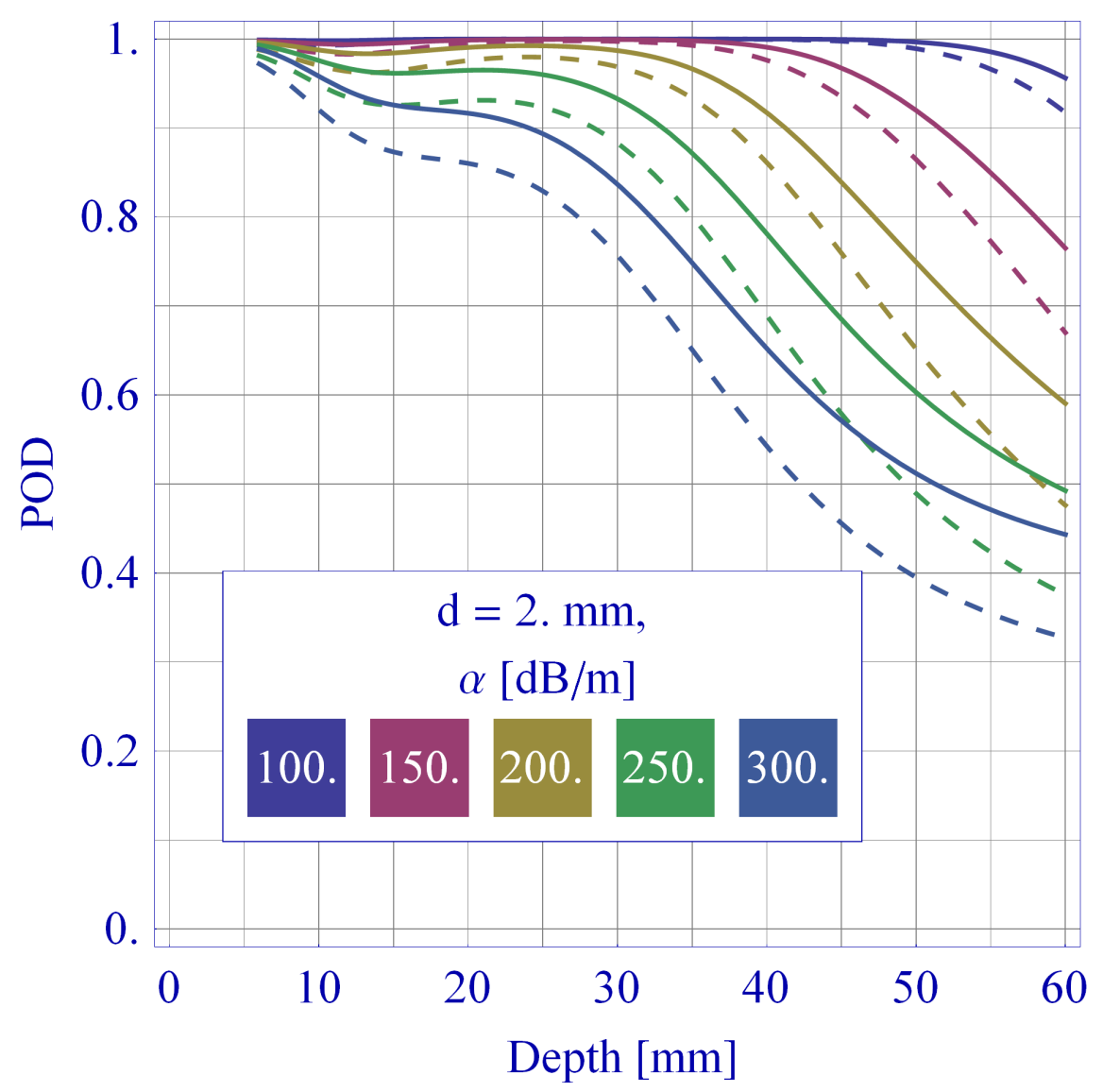

Figure 4.18: POD curves with lower 95\% confidence bounds for constant $d$ POD curves (solid lines) with lower $95 \%$ confidence bands (dashed lines) of the FBH with diameter $\mathrm{d}=2.0 \mathrm{~mm}$ as a function of depth, for different attenuation coefficients

From the $\hat{a}-a_{M P}$ diagram, the POD is calculated. In figure 4.18 the POD curves with lower 95\% confidence bands, expressed as a function of depth for a FBH with diameter $2 \mathrm{~mm}$ and different attenuation coefficients are shown. The POD of the flaw is decreasing as the attenuation coefficient of the surrounding material increases. As expected, this effect is more noticeable at higher depths due to the exponential increase of attenuation with the depth. Also, the local maximum of the amplitude of the focused 
beam is decreasing and shifting towards shallower depths.

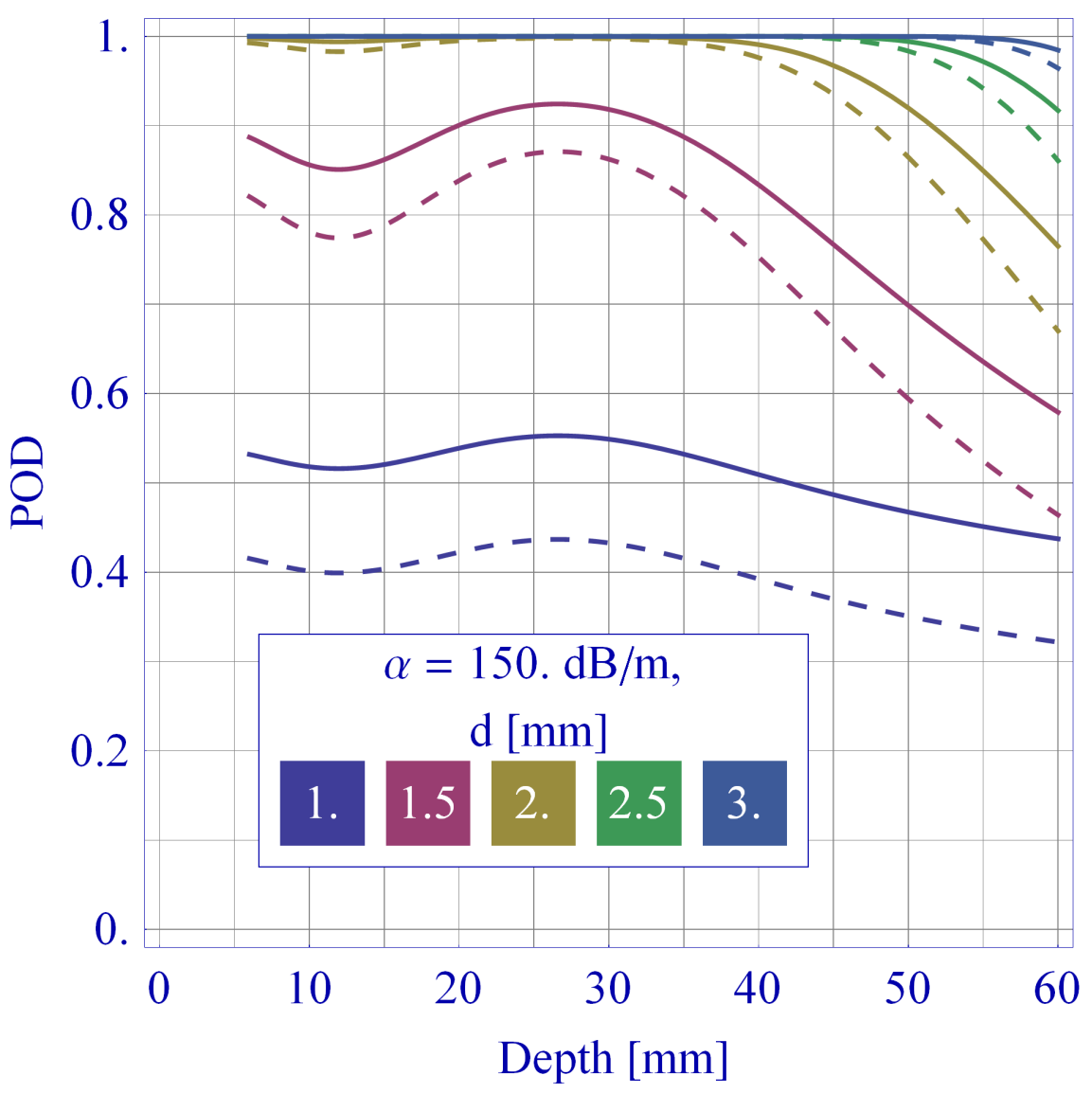

Figure 4.19: POD curves with lower 95\% confidence bounds for constant $\alpha$ POD curves (solid lines) with lower $95 \%$ confidence bands (dashed lines) in material with the attenuation coefficient $\alpha=150 \mathrm{~dB} / \mathrm{m}$ as a function of the depth, for flat-bottom holes with different diameters

In figure 4.19 the POD curves with lower 95\% confidence bands, expressed as a function of depth for a FBH in material with the attenuation coefficient $150 \mathrm{~dB} / \mathrm{m}$ and different diameters are shown. The POD of the flaw is decreasing as the size of the flaw is decreasing, providing the attenuation is the same. 
4. STUDY I 
5

\section{Study II}

The capability of the NDT system to detect flaws is quantified with the POD curve. If the system is adequate to find flaws of a certain size, is based on one number that is read from the POD curve, as for example $a_{90 / 95}$. When the flaw depth is also an essential influencing parameter, it would be more suitable to plot a POD as a scatter plot over a cross section of the inspected component.

Several inspections of the same component are planned to fulfil the requirement to inspect $100 \%$ of the volume. With a POD analysis, the capability of only one inspection can be estimated. To establish the overall capability to detect flaws, when all inspections are used together, a POD data fusion is needed.

\subsection{Volume POD and POD data fusion}

\subsubsection{Materials and methods}

\section{Flaw depth as an influencing parameter}

When the depth of the flaw, i.e. the distance from the inspection surface, is an influencing parameter for the POD, as for example in ultrasonic inspection of thick parts with high attenuation in the material, by using multi-parameter POD model the POD can be calculated and expressed, among other parameters, also as a function of the flaw depth:

$$
P O D=P O D(z)
$$




\section{STUDY II}

where $z$ is the depth of the flaw or the distance of the flaw from the surface from which the part is inspected. Using the simulation of the inspection process, the response is calculated in the parameter size range of interest. Next, the flaws with varying parameters in the same size range are manufactured in the test object. The test object is inspected and responses recorded. The $\hat{a}-a_{M P}$ diagram can now be created. From this diagram, the $\operatorname{POD}\left(a_{M P}\right)$ is calculated from which the POD curves as a function of individual parameters can be calculated in the next step. The $P O D(z)$ diagram shows a dependence of the POD on the depth. Calculating the $\operatorname{POD}(z)$ for adjacent points on the part, a POD contour diagrams can be created. When overlaid on the part geometry we refer to it as the volume POD diagram.

To inspect the material fully, sometimes more than one inspection is used. If the POD of those inspections are expressed as the function of the depth, multiple volume POD diagrams can be created. For independent inspections, the overall probability that the flaw will be missed can be written as:

$$
\left(1-P O D_{\text {overall }}\right)=\prod_{i=1}^{n}\left(1-P O D_{i}\right)
$$

where $n$ is a number of individual inspections. The equation can be rewritten as:

$$
P O D_{\text {overall }}=1-\prod_{i=1}^{n}\left(1-P O D_{i}\right)
$$

Using this equation for POD data fusion from multiple inspections, the overall POD in every point of the volume of the part can be calculated. Overlaying the overall POD diagram on the part geometry, the overall volume POD diagram is created. Calculating the POD of the maximum allowable flaws and checking if the lower $95 \%$ confidence band has the POD of $90 \%$ or higher and plotting it in the volume diagram, the adequacy of

the inspection system can be checked. Areas where the NDT system achieves desired level of POD and where not, should be clearly visible.

\subsubsection{Experimental set-up}

\section{Ultrasonic Inspections of the Thick Copper Component}

The volume POD model and the fusion of the POD data were applied on the UT data from the inspection of the canister copper lid. The lid is made of highly pure copper, 
its diameter is approx $1 \mathrm{~m}$ and has a thickness of $55 \mathrm{~mm}$ in the thinnest part and 195 $\mathrm{mm}$ in the thickest part. The requirement is to inspect $100 \%$ of the material.

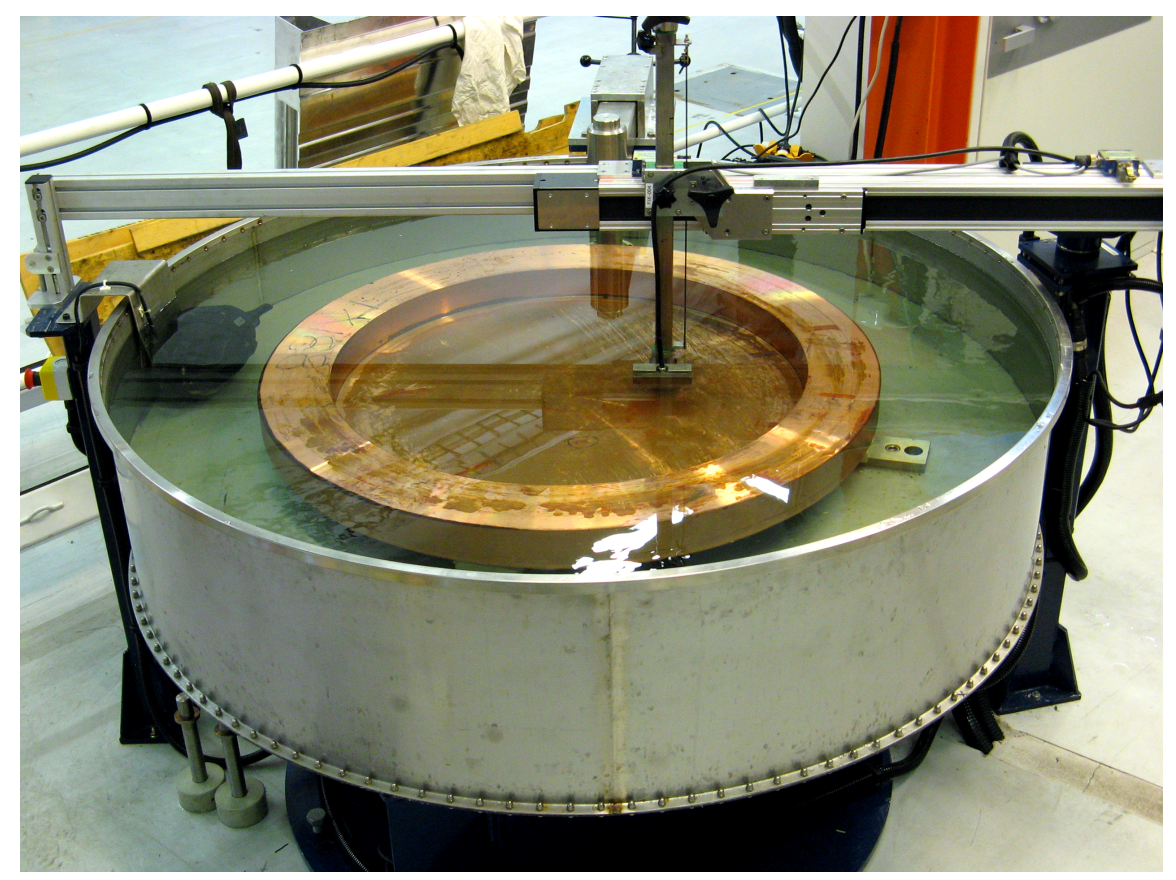

Figure 5.1: Rotating water tank - Copper lid immersed in a rotating water tank, ready for inspection

Because of the thickness, material and geometry, this requirement makes a design of the inspection system a challenging task. It is not possible to inspect the whole volume with just one method. The inspection system needs to achieve a required level of the POD of the postulated flaws in the whole material. Still, the inspection should be performed in a time and cost effective manner.

There are many inspection methods available for NDT system designers to choose from, but after some considerations it was decided to use an automated phased array ultrasonic system. Through the computer controlled excitation of the individual elements of the phased array probe, ultrasonic focused beam can be generated with the possibility of modifying the beam parameters such as angle, focal distance and focal spot [23]. This can simplify the inspection of components with complex geometry. This way, by using the same hardware - which will keep the inspection system simple - it is possible to change the parameters of the beam to optimize its characteristics, depending on the inspections depth - which will ensure a high POD. For the inspection the lid 


\section{STUDY II}

is immersed in the water tank which can fully rotate. The lid is shown in figure 5.1 . By means of a mechanical arm, the probe is brought into the position for the inspection. The lid is rotated $360^{\circ}$ while the inspection data is recorded and saved for later evaluation. After the full rotation, the probe is moved to the adjacent position and everything is repeated until the whole surface being inspected is covered. To make sure that both horizontally and vertically oriented flaws will be detected, the lid will be inspected from the top and from the outer, side surface. For the detection of flaws nearer to the surface, the technique with the water buffer is used, whereas for the detection of deeper flaws, the contact technique is used. For inclined flaws, inspections with the angled beam are used. Even if the same probe is used for all inspections, whenever the settings of inspection are changed it is considered as a different inspection. In the terminology of data integration from different sensors, each inspection can be regarded as if performed by a different sensor.

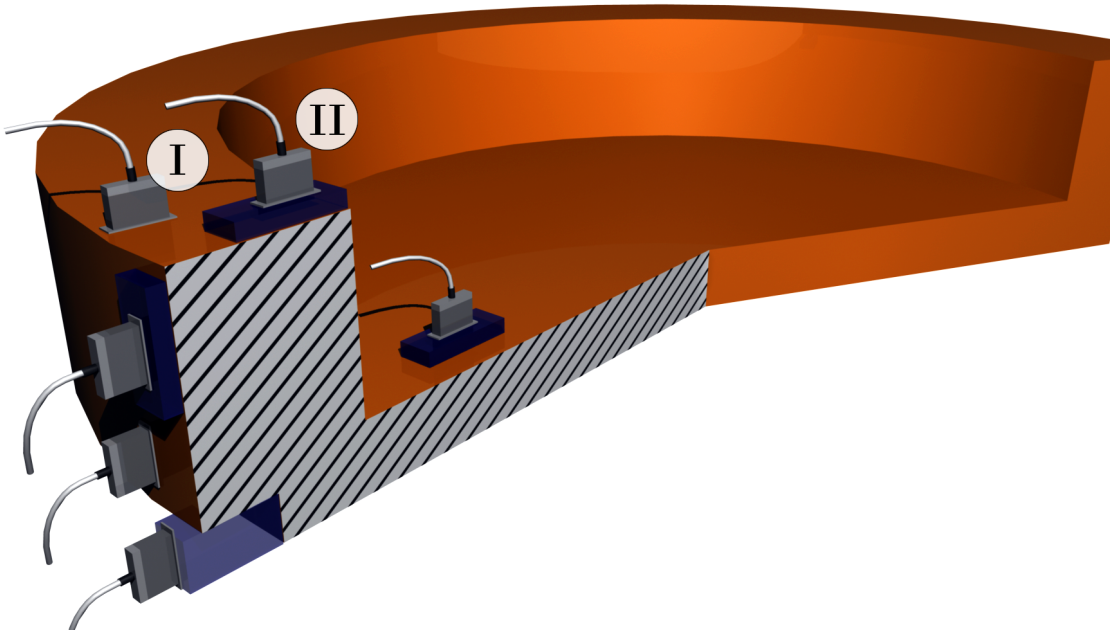

Figure 5.2: Transducer positioning for the lid inspection - Positioning of the transducer on the lid for different inspections

For the model demonstration, inspection data from two inspections have been used. The position of the probe for different inspections is illustrated in figure 5.2. The arrangement $I$ is an immersion inspection intended for the detection of horizontally oriented flaws in shallower depths, from the top surface of the lid. The arrangement $I I$ is a contact technique, intended for detection of horizontally oriented flaws in the deeper parts of the lid. 
As important influencing parameters on the POD, the type of the flaw, its size (area), depth (distance from the inspection surface) and orientations (inclination angle) were selected. The type of the flaw considered was a planar flaw. To investigate the capability of the ultrasonic system to detect flaws, artificially manufactured flat bottom holes were used. The range of interest for the flaw was $1-5 \mathrm{~mm}$ in radius, $5-200 \mathrm{~mm}$ in depth and $0-90^{\circ}$ in inclination angle.

The simulation used for the numerical calculation of the response was the BAM software based on the point source synthesis method, described in section 2.6. The reflection echo coming from the FBH reflector was calculated in the size range of the influencing parameters. In the same range, a number of FBHs were manufactured in the test object. The object was inspected and inspection data recorded. Maximum amplitudes were noted at the FBHs' locations. For each inspection the measured amplitude was plotted against the amplitude calculated by the simulation, creating the $\hat{a}$ versus $a_{M P}$ diagram from which the POD curve as a function of multi-parameter $a_{M P}$ was calculated. Having all parameters except the depth constant, the POD curve as a function of depth was created. This is needed for the construction of the volume POD diagram that is overlaid on the geometric drawing of the inspected part. Finally, the POD data fusion from different inspections is performed according to equation 5.3 .

\subsubsection{Results and discussion}

The POD curves and lower 95\% confidence bands for both inspection $I$ and $I I$, calculated with a multi-parameter model and expressed as a function of depth are shown in figures 5.3 and 5.4. In both diagrams, the curves that run from 6 to $60 \mathrm{~mm}$ depth are from the inspection $I I$ and curves that run from 45 to $195 \mathrm{~mm}$ depth are from the inspection $I$. There is an overlap of the curves from 45 to $60 \mathrm{~mm}$.

Figure 5.3 shows the POD (solid lines) and lower 95\% confidence band (dashed lines) for FBHs with $3 \mathrm{~mm}$ diameter and 0,3 and $6^{\circ}$ inclination angle. The angle $\beta$ is an angle of the FBH normal and the main axes of the lid. The FBHs with a $0^{\circ}$ angle are horizontal. The ultrasonic sound wave from both inspections $I$ and $I I$ will encounter the flaw normal to its surface, which will produce a maximum reflection of the wave. Therefore, the POD will drop as the angle $\beta$ increases, as it is shown in the

diagram. The change of the POD with a depth needs a deeper explanation. From the conventional POD model, one is accustomed that the POD is a monotonic function 


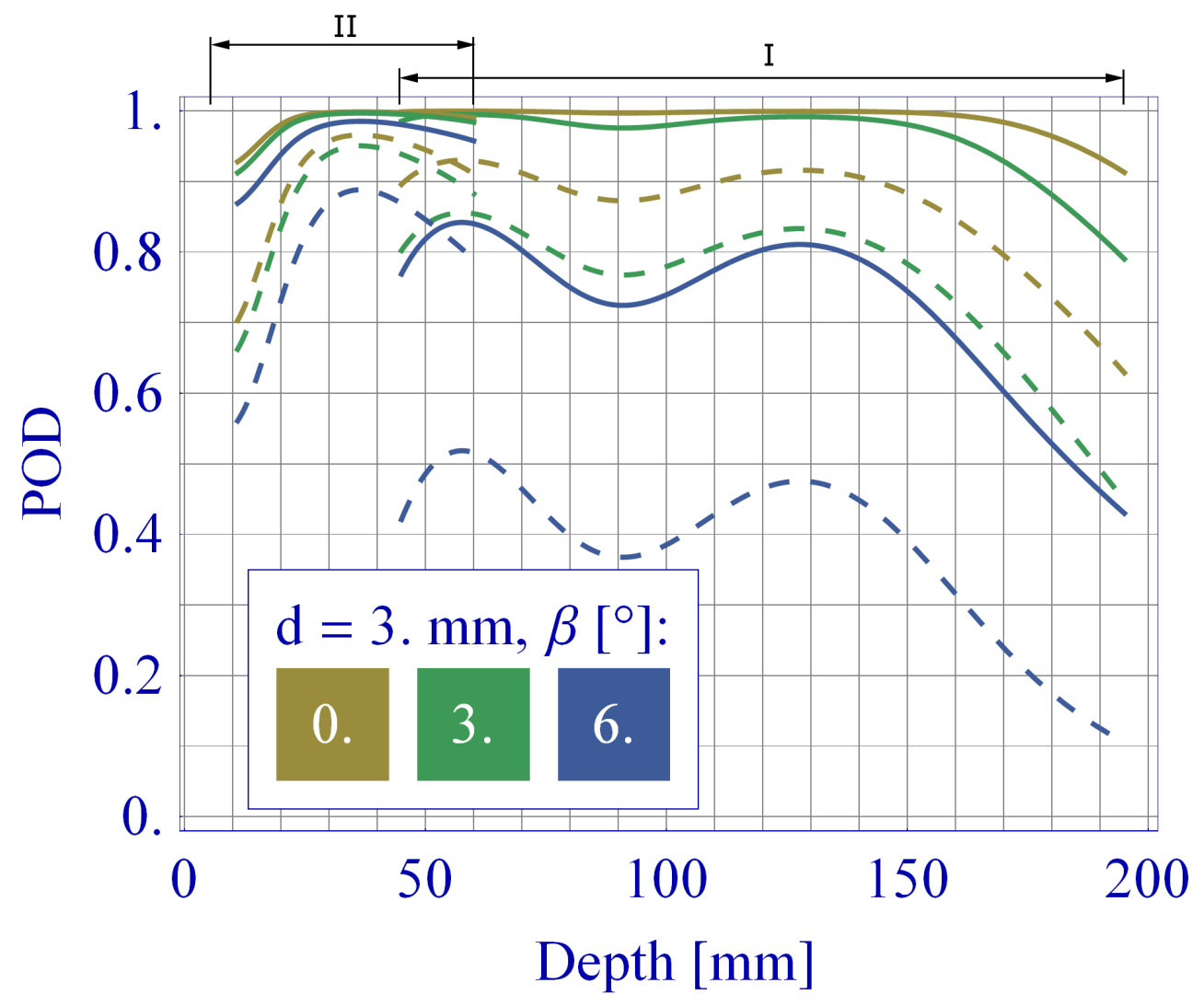

Figure 5.3: POD as a function of depth and angle for constant diameter - POD (solid lines) and lower 95\% confidence band (dashed lines) expressed as a function of depth, for FBHs with $3 \mathrm{~mm}$ diameter and 0,3 and $6^{\circ}$ inclination angle 
that grows with the flaw size from 0 to 1 . In figure 5.3 however, both rising and falling of the POD function with depth can be observed. The reason is that the POD is proportional to the amplitude of the reflected wave. The amplitude of the ultrasonic wave will change as it propagates through the material due to the attenuation in the material but also due to the sound beam properties determined by the focal laws used for the generation. The effect on the POD can be observed in a diagram. High POD for the inspection $I I$ is in depths around $30 \mathrm{~mm}$ and for the inspection $I$ in the depths around $130 \mathrm{~mm}$. These depths correspond to the focal depths of the inspections. The POD in shallower or deeper depths will be usually smaller and change as the reflected amplitude changes due to the attenuation and due to the properties of the wave.

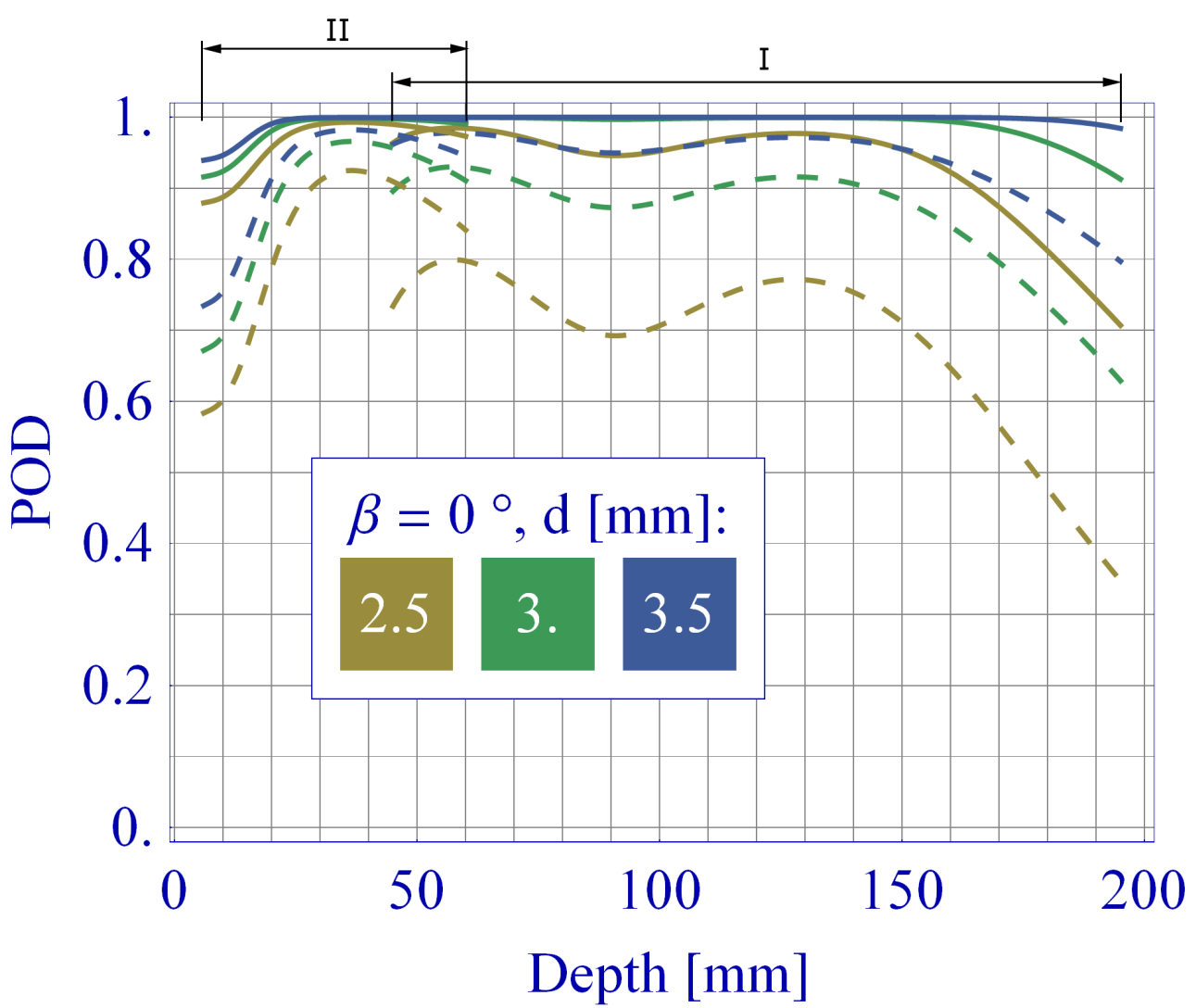

Figure 5.4: POD as a function of depth and diameter for constant angle - POD (solid lines) and lower 95\% confidence band (dashed lines) expressed as function of depth, for FBHs with $0^{\circ}$ inclination angle and 2.5, 3.0 and $3.5 \mathrm{~mm}$ diameter

Figure 5.4 shows the POD (solid lines) and lower 95\% confidence band (dashed 


\section{STUDY II}

lines) for FBHs with $0^{\circ}$ inclination angle and 2.5, 3.0 and $3.5 \mathrm{~mm}$ diameter. It can be observed in the diagram that the POD will drop with a decrease of size of the reflector. FBHs with a smaller diameter have a smaller POD than those with a larger diameter. The change of the POD with the depth is similar as in figure 5.3. High POD is achieved at the depths of the focus of the ultrasonic focused beam. The POD changes as the amplitude of the wave changes due to the influence of attenuation and properties of the sound beam.

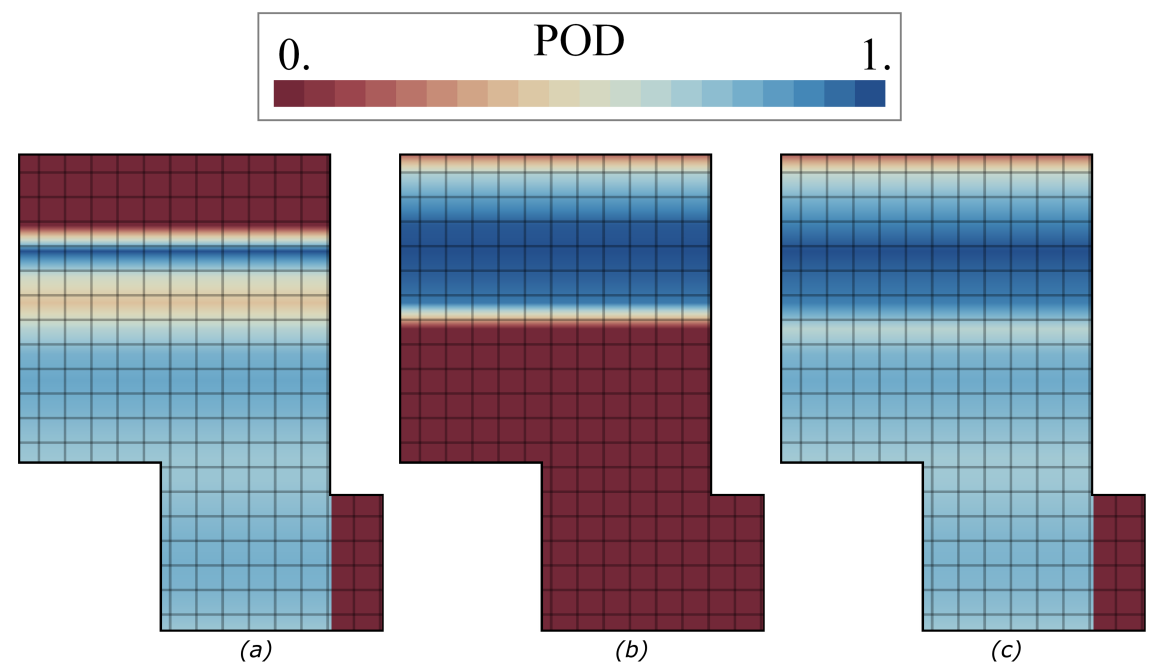

Figure 5.5: Volume POD - Volume POD diagram of the inspection I (a), II (b) and fusion of the two (c), for the FBH with $\beta=0^{\circ}$ and $d=2.5 \mathrm{~mm}$

The diagrams shown in figures 5.3 and 5.4 , where the POD is shown as a function of the depth, can be seen as a POD of the single ultrasonic beam or of the one A-scan. If the POD of many adjacent scans are composed together, the spatial distribution of the POD is created. This can be seen as the POD of the B-scan. If these diagrams are overlaid on the component geometry, the volume POD diagram is created, as shown in figure 5.5. In figure 5.5 (a) the volume POD of the inspection $I$ is shown, in (b) the volume POD of the inspection $I I$ is shown and in (c) the fused volume POD according to equation 5.3 or the overall volume POD diagram is shown. The POD is displayed for the FBH with $\beta=0^{\circ}$ and $d=2.5 \mathrm{~mm}$. The same way, the POD can be displayed for any values of influencing parameters. The POD diagram of the inspection $I I$ covers the volume between $6-60 \mathrm{~mm}$, whereas the POD diagram from the inspection $I$ covers the 
volume between 45-195 $\mathrm{mm}$ (same as in diagrams depicted in figures 5.3 and 5.4 . The benefit of the fusion of the POD data is a more comprehensive picture of the POD than what would be obtained from a single inspection, in the region where both inspections inspect the same volume of material, i.e. from $45-60 \mathrm{~mm}$.

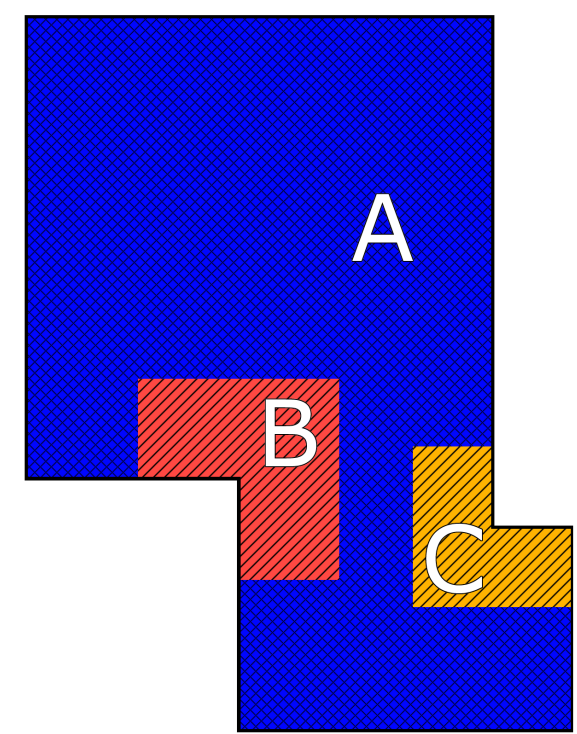

(a)

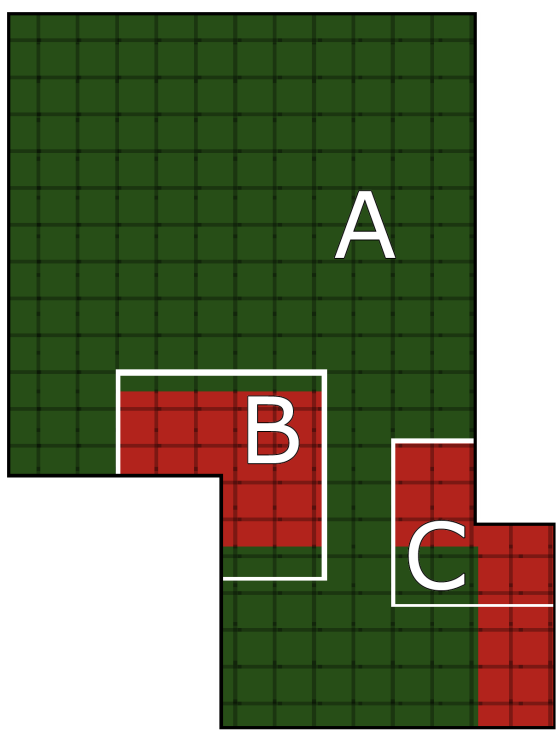

(b)

Figure 5.6: Acceptance criteria - Cross section of the lid with indicated regions with the different maximal allowable flaw size(a) and regions where the lower $95 \%$ confidence limit is not larger than the $90 \%$ probability of detection (b)

By plotting the lower $95 \%$ confidence band values instead of the POD, the diagram can be compared with the values of the maximum acceptable flaw size. Maximum acceptable flaw size can change, depending on the location of the flaw within the component. Suppose that there are three distinctive regions in the cross-section of the lid with different maximum acceptable flaw size as shown in figure 5.6 (a). In the given example, maximum allowable flaw size with $\beta=0 \mathrm{deg}$ is $3 \mathrm{~mm}$ in the region $\mathrm{A}$ and $2.5 \mathrm{~mm}$ in the regions $\mathrm{B}$ and $\mathrm{C}$. In the diagram shown in figure 5.6 (b), regions where the lower $95 \%$ confidence band of the maximum acceptable flaw is higher than $90 \%$ are plotted in dark green and when lower then $90 \%$ in light red. From this diagram, it is now easy to identify regions of the volume that are not adequately inspected and where additional inspections are needed. In the given examples, red areas in the region $\mathrm{B}$ and $\mathrm{C}$ indicate to low POD and need to be inspected additionally. An additional 


\section{STUDY II}

inspection from the upper surface, with the focal depth of the beam set to the depth of the light-shaded areas should solve the problem. To optimize the inspection, it is not necessary to perform the inspection across the whole surface, but only in the region directly above the regions $\mathrm{B}$ and $\mathrm{C}$. Once the inspection is performed, the volume POD diagram of the additional inspection should be created and the POD data fusion with other inspections performed. Once the whole inspection volume is shown in green, the required level of POD is achieved in the whole component. 


\section{6}

\section{Conclusions}

\subsection{Summary of contributions}

\subsubsection{Multi-parameter POD model}

The multi-parameter POD model developed in this thesis shows a new way to express the POD of the NDT system. Using a computer modelling of the inspection process, as well as experimentally measured data, the POD is calculated and expressed as a function of several parameters. These parameters are chosen from the set of parameters that influence the POD. They are established to have the greatest influence on the POD in the observed inspection case and are known as essential influencing parameters. In the conventional signal response model, on which multi-parameter builds upon and expands, the POD is expressed as a function of the one most influencing parameter. In most of the application cases, this parameter is a flaw size.

The POD curve quantifies the capability of the NDT system to detect flaws. The conventional POD curve quantifies this capability in relation to the flaw size. By determining the minimum size of the flaw that the system can still reliably detect and by comparing it with the size of the flaw that is critical for the structural integrity, it can be determined if the inspection system is adequate for the inspection task. The multiparameter POD quantifies the capability of the NDT system to detect flaws regarding any of the influencing parameters included in the analysis. The suitability of the NDT system for the inspection task is evaluated by comparing the value of the parameter of reliably detected flaw with the critical value of that parameter. For example, if the essential influencing parameters are flaw depth extension and flaw length, the POD is 


\section{CONCLUSIONS}

calculated as a function of both parameters. To demonstrate that the NDT system is suitable for the inspection task, the depth extension of reliably detected flaw has to be smaller than the critical depth extension and the length of reliably detected flaw has to be smaller than the critical flaw length. Only if both these conditions are satisfied, it can be confirmed that the NDT system is fit for purpose. If the POD is expressed as a function of the flaw size only, whereas other parameters have as large influence on the POD, it would lead to an error when establishing inspection system's fitness for purpose. Either the NDT system established as fit for purpose would not reliably detect all flaws which are critical for the structural integrity, or the system being fully capable to reliably detect critical flaws would be regarded as inadequate.

The multi-parameter model was applied on the ultrasonic inspection data. The cast iron component with embedded semi-elliptical crack-like flaws was inspected with the ultrasonic TRL system. The length and depth extension of the flaw were chosen as essential influencing parameters for the multi-parameter analysis. The response amplitudes from the flaws with different depth extensions and lengths manufactured in the test specimen, were measured in the experiment. The response amplitudes were also calculated using a computer simulation. In the next step, the POD of the flaw was calculated as a function of those two parameters. For comparison, the POD was also calculated using conventional signal response analysis, as a function of the area of the flaw.

The POD curves resulting from the multi-parameter analysis show dependence on both flaw depth extension and flaw length. The value of the reliably detected flaw are therefore determined for both parameters. Since the severity of the flaw with the respect to structural integrity of the inspected component is also expressed with the critical value of those two parameters, values are directly compared to determine if the NDT system is capable of reliably detecting critical flaws. This is the case only if the parameter values of reliably detected flaws are smaller than the critical values of both parameters. This has been proven to be the case for the evaluated inspection system.

The POD curve from the conventional analysis shows a dependence of the POD on the flaw area. The NDT system is capable of detecting critical flaws if the area of the reliably detected flaw is smaller than the area of the critical flaw. Since the dimensions of the critical flaw are given by its depth extension and length, the area of the critical flaw is calculated from these two parameters. When comparing the area of the reliably 
detected flaw and the area of the critical flaw the observed inspection system has been proven to be adequate.

The results from both models show that the investigated NDT system is capable of reliably detecting all critical flaws. However, when comparing the POD curves calculated with the multi-parameter model with the curve calculated in a conventional way, an important characteristic has been observed: in the POD transition region (region where the POD changes from 0 to 1 ) flaws that have the same area will have different POD depending on their depth extension and length. The multi-parameter model will account for this phenomenon, whereas the conventionally calculated POD curve will make no distinction between flaws having the same area but different depth extension and length, consequently either overestimate or underestimate the capability of the NDT system to detect flaws.

The POD curves calculated with the multi-parameter model show the influence of each parameter on the POD and provide better understanding of the detection process. The model will find application in those inspection cases where several parameters have an important influence on the POD of the flaw. Application of the multi-parameter model will be also advantageous in those cases where other parameters beside the flaw size determine the severity of the flaw for the structural integrity of the component.

\subsubsection{The POD expressed as a function of the attenuation coefficient}

The microstructure of metallic components depends on the manufacturing process and the treatments that the component has undergone. The grain size, the grain size distribution and the grain orientation can vary within the component and from component to component. The microstructure is a principle factor that will influence the attenuation of the ultrasonic sound wave through the mechanism of scattering on the grain boundaries. As a consequence, the ultrasonic attenuation will be one of the essential influencing parameters on the POD of the ultrasonic NDT system. This is especially the case if the thick components are inspected, because of the longer path that the sound wave travels making the attenuation of the wave more prominent. Two flaws of the same characteristics will have a different POD depending on the microstructure of the surrounding material. Therefore, it is important to calculate and express the POD as a function of ultrasonic attenuation. 


\section{CONCLUSIONS}

In the experiment described in the thesis flaws with the simple geometry (flatbottom holes) and variable diameter and depth were introduced in the copper specimens with variable microstructure. The specimens were inspected with the ultrasonic phased array system with the focused beam. Response amplitudes were measured as well as calculated by the simulation software. The POD curves expressed as a function of attenuation coefficient were calculated.

The analysis showed an expected decrease of the POD with an increase in attenuation. The decrease is more noticeable at larger depths because of the exponential increase of attenuation with the depth. One more interesting phenomenon has been observed. This is the theoretical depth of the focal point of the focused ultrasonic beam which is shifting towards the probe with increasing attenuation.

The practical application of the analysis results could be used as an acceptance criterion for the component. Knowing the capability of the ultrasonic system that will be used for the inspection and knowing the properties of the flaws being critical for the structural integrity, the maximum allowable attenuation in the component can be set. By simply measuring the attenuation in a component it can be decided if the microstructure of the component will allow the NDT system to be successful in detection of critical flaws and if the component can hence be allowed to enter into service. Also, the focusing of the beam can be optimized by compensating for the shift of the focal point due to the attenuation of the sound wave, so that the maximum detectability is achieved in the desired depth.

\subsubsection{Spatial representation of POD data and a POD data fusion}

A typical POD curve shows the dependence of the POD on one parameter. If this parameter is a distance of the flaw from the inspection surface, a POD curve shows a change of the POD with the distance. If the sensor is moved along the inspection surface during the inspection, a POD curve can be constructed for every position of the sensor on the inspection surface. All these POD curves can be summed-up in one 2-D contour diagram. This diagram overlaid on the geometry of the inspected component illustrates the capability of the NDT system to detect flaws in the volume of the component more clearly than the POD curve. This type of the diagram is named the volume POD diagram. 
Due to the geometrical complexity of some components, multiple sensors are used to reliably inspect the whole volume of the component. Increasing the number of the sensors will also increase the POD, but the complexity of the NDT system will also increase. The more complex the inspection system, the harder it will be to determine if it is capable to detect all critical flaws. The POD diagrams for individual sensors show the capability of individual sensors to find a flaw but not when all sensors are applied together. Yet the aim is to find the POD of the whole NDT system, when all sensors are applied together. The fusion of the POD data is needed to achieve this goal. The volume POD diagram facilitates the data fusion for each point in the volume of the component. The resulting diagram shows the POD of the flaw when the component is inspected with all sensors - an overall POD.

In the experiment described in the thesis, data from the two ultrasonic phasedarray probes inspecting the same component were used to create two volume POD diagrams. The data fusion of the data was performed resulting in the overall volume POD diagram.

The advantage of this diagram is that it shows areas where the overall POD lies below the desired level. These areas need additional inspection to increase the level of POD. It also shows areas where inspections with different sensors are redundant. The number of sensors inspecting these areas can be reduced, decreasing the time needed for the inspection without decreasing the desired level of POD. This way the POD diagram can be used as an optimisation tool of the NDT system, and not only as a measure of its capability to detect flaws. Conclusions of the NDT reliability project of the Swedish final repository for spent nuclear fuel lead to the reduction of the number of canister inspections and to the simplification of geometry of the canister to facilitate time-efficient, cost-effective and reliable inspection.

\subsection{Recommendations for future work}

\subsubsection{Application of the multi-parameter model on the real flaws}

The multi-parameter model was tested on the inspection data from artificial flaws. Artificial flaws have simple geometry and can be manufactured relatively easy in the test specimen in various shapes, sizes, depths and orientations. Varying controllable parameters of the flaws and measuring their response amplitudes, an extensive reliability 


\section{CONCLUSIONS}

analysis can be performed. However, most of the real flaws occurring in the engineering structures are of complex geometry. In some cases there is a possibility to correlate the response of the real flaws with the artificially manufactured ones. This correlation is often unfortunately hard or even impossible to find and the reliability analysis has to be performed on the large number of the real flaws. To confirm the multi-parameter model in the real-world applications, it has to be tested on the inspection data coming from the real flaws.

For example, pores, the typical non-continuous casting flaws, can be described by pore to surface distance and pore dispersion coefficient [106]. It would be interesting to express the POD of pores as a function of those (and possibly other) parameters.

To successfully test the multi-parameter model on the inspection data from the real flaws, there are several conditions that need to be met. There has to be sufficient number of flaws available with varying essential influencing parameters in the region where the POD changes from 0 to 1 . The response from these flaws has to be measured. And finally, the simulation software capable to calculate the theoretical response of the flaws with changing parameters has to be available.

\subsubsection{Replacement of the maximum amplitude with a more compre- hensive response signal characteristic}

In all calculations a maximum measured amplitude of the response signal was taken as the signal response $\hat{a}$. It was assumed that the amplitude of the response signal reflects the size of the flaw, i.e. the larger the flaw, the larger the response amplitude. This assumption proved to be valid for ultrasonic inspections of the flat flaws with simple geometry, smaller than the ultrasonic beam cross section at the flaw location. In the performed experiments, inspection data from flat-bottom holes and semi-elliptical notches supported this assumption and showed the correlation between the maximum amplitude and the flaw size. In the industrial applications, flaws in general will not be flat and will have a more complex geometry. The maximum amplitude might not reflect the size of the flaw, i.e. a smaller flaw can under circumstances produce larger signal response amplitude than a larger flaw. Without the correlation between maximum response amplitude and the flaw size, the signal response model can not be used to calculate the POD. 
There is more information contained in the inspection data from the ultrasonic system than just the maximum response amplitude. It is necessary to extract from this data a property of the response that is correlated with the flaw size. This property is likely to be different for different types of the flaws. One possible approach is an integration of the signal amplitudes over the flow projection area, as investigated in [107.

\subsubsection{Application of the multi-parameter model on other NDT meth- ods}

The multi-parameter model was applied only on the data originating from different ultrasonic inspection systems. Both the analysis of the phased-array data and the TRL data yielded good results. There is no visible obstacle to apply the model on inspection data from systems operating on different physical principles. Three basic conditions had to be met. A number of flaws, whose essential parameters vary in magnitude in the region where the POD is changing from 0 to 1 , has to be available. The sufficient number will depend on the number of essential influencing parameters. The signal response from these parameters needs to be measured experimentally. A simulation model, capable of expressing the theoretical response of the used inspection system on the essential influencing parameters, needs to be available. Once the responses are measured and dependence on the influencing parameters calculated, the POD analysis is straightforward. Also, the overall POD in the components inspected with several different NDT methods can be calculated by performing a data fusion of the POD data from different sensors. Since the POD data has the same form regardless of the operating principle of the sensor, the fusion can be performed the same way as it has been performed for different ultrasonic probes [108]. 
6. CONCLUSIONS 


\section{Appendix A}

\section{Copper specimens}

In this appendix two copper specimens used in the experiments are described. Figure A.1 and A.2 show technical drawing of the specimen BAM1 and BAM1B, respectively. The locations of the each FBH is indicated in the drawing. Next to the each flat-bottom hole, its diameter and depth are given in parentheses. Every flat-bottom hole is identified with a unique ID number, shown in the first column in table A.1. In the second and third column, diameter and depth of the FBH are given respectively. In the fourth and fifth column, FBH's $\mathrm{x}$ and $\mathrm{y}$ distance from the lower left corner of the specimen (as indicated in the drawings) are given. In the last, sixth column, the thickness of the specimen at the FBH's position is given.

Measured signal response amplitudes are given in table A.2. First column displays the unique ID number of the FBH. In the second column the amplitude of the backwall echo in the proximity of the FBH is displayed. The third column gives the backwall echo amplitude exactly at the position of the FBH. A maximum reflected amplitude at the position of the FBH is displayed in the fourth column. In the fifth column the average noise level in the proximity of the FBH is given and in the last, sixth column, a signal-to-noise ratio is shown. 


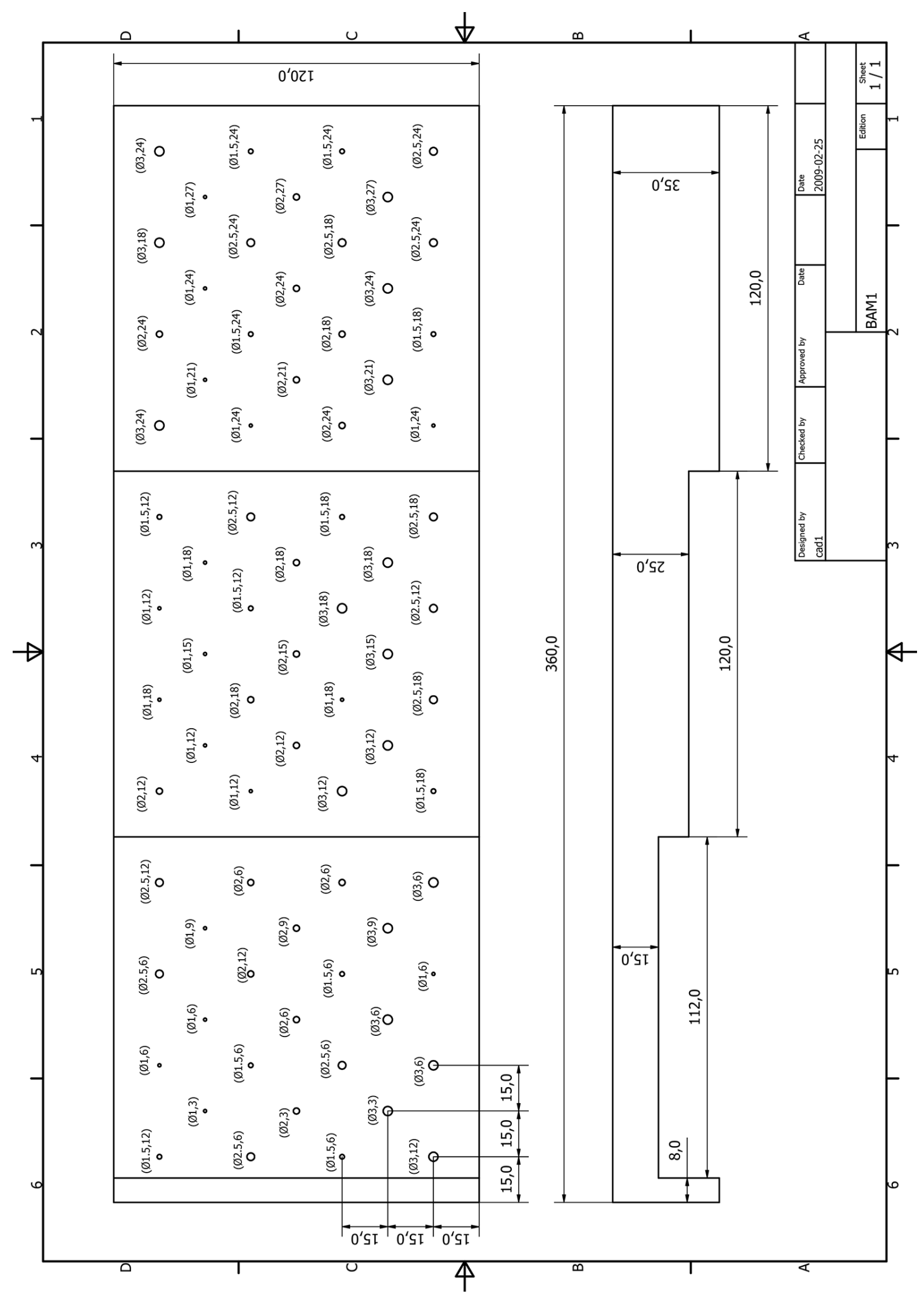

Figure A.1: Copper specimen BAM1 - Technical drawing of the specimen with indicated flat-bottom holes positions, diameters and depths (in paranthesis) 


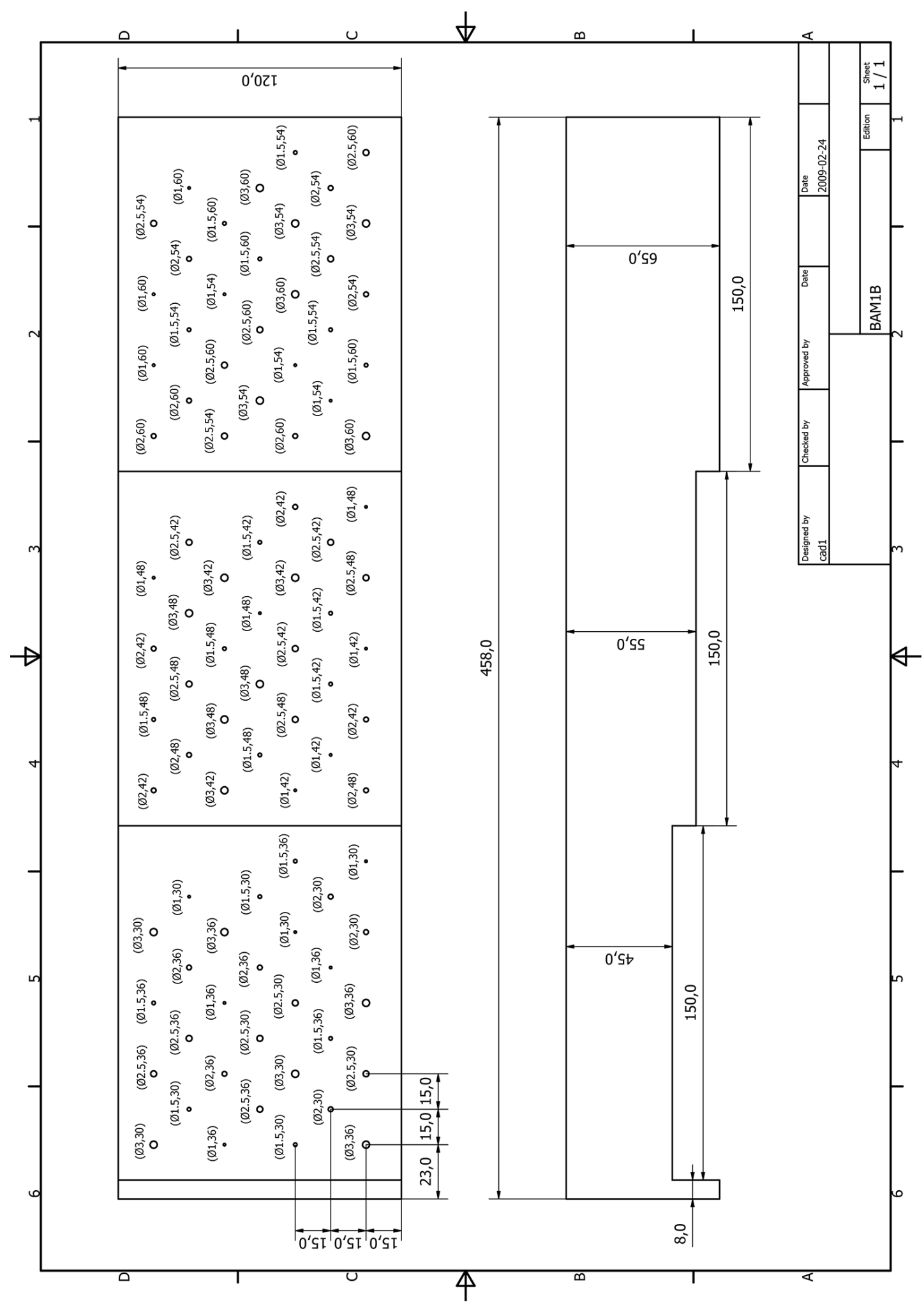

Figure A.2: Copper specimen BAM1B - Technical drawing of the specimen with indicated flat-bottom holes positions, diameters and depths (in paranthesis) 


\section{A. COPPER SPECIMENS}

Table A.1: Identification of the flat-bottom holes with their diameter $d$, depth $z$ and position $x$ and $y$ on the specimens and a specimen thickness $t$

\begin{tabular}{|c|c|c|c|c|c|}
\hline ID & $\mathrm{d}[\mathrm{mm}]$ & $\mathbf{z}[\mathbf{m m}]$ & $\mathbf{x}[\mathbf{m m}]$ & $\mathbf{y}[\mathbf{m m}]$ & $\mathbf{t}[\mathbf{m m}]$ \\
\hline 1 & 3.0 & 12.0 & 15.0 & 15.0 & 15.0 \\
\hline 2 & 3.0 & 6.0 & 45.0 & 15.0 & 15.0 \\
\hline 3 & 1.0 & 6.0 & 75.0 & 15.0 & 15.0 \\
\hline 4 & 3.0 & 6.0 & 105.0 & 15.0 & 15.0 \\
\hline 5 & 3.0 & 3.0 & 30.0 & 30.0 & 15.0 \\
\hline 6 & 3.0 & 6.0 & 60.0 & 30.0 & 15.0 \\
\hline 7 & 3.0 & 9.0 & 90.0 & 30.0 & 15.0 \\
\hline 8 & 1.5 & 6.0 & 15.0 & 45.0 & 15.0 \\
\hline 9 & 2.5 & 6.0 & 45.0 & 45.0 & 15.0 \\
\hline 10 & 1.5 & 6.0 & 75.0 & 45.0 & 15.0 \\
\hline 11 & 2.0 & 6.0 & 105.0 & 45.0 & 15.0 \\
\hline 12 & 2.0 & 3.0 & 30.0 & 60.0 & 15.0 \\
\hline 13 & 2.0 & 6.0 & 60.0 & 60.0 & 15.0 \\
\hline 14 & 2.0 & 9.0 & 90.0 & 60.0 & 15.0 \\
\hline 15 & 2.5 & 6.0 & 15.0 & 75.0 & 15.0 \\
\hline 16 & 1.5 & 6.0 & 45.0 & 75.0 & 15.0 \\
\hline 17 & 2.0 & 12.0 & 75.0 & 75.0 & 15.0 \\
\hline 18 & 2.0 & 6.0 & 105.0 & 75.0 & 15.0 \\
\hline 19 & 1.0 & 3.0 & 30.0 & 90.0 & 15.0 \\
\hline 20 & 1.0 & 6.0 & 60.0 & 90.0 & 15.0 \\
\hline 21 & 1.0 & 9.0 & 90.0 & 90.0 & 15.0 \\
\hline 22 & 1.5 & 12.0 & 15.0 & 105.0 & 15.0 \\
\hline 23 & 1.0 & 6.0 & 45.0 & 105.0 & 15.0 \\
\hline 24 & 2.5 & 6.0 & 75.0 & 105.0 & 15.0 \\
\hline 25 & 2.5 & 12.0 & 105.0 & 105.0 & 15.0 \\
\hline 26 & 1.5 & 18.0 & 135.0 & 15.0 & 25.0 \\
\hline 27 & 2.5 & 18.0 & 165.0 & 15.0 & 25.0 \\
\hline 28 & 2.5 & 12.0 & 195.0 & 15.0 & 25.0 \\
\hline 29 & 2.5 & 18.0 & 225.0 & 15.0 & 25.0 \\
\hline 30 & 3.0 & 12.0 & 150.0 & 30.0 & 25.0 \\
\hline 31 & 3.0 & 15.0 & 180.0 & 30.0 & 25.0 \\
\hline 32 & 3.0 & 18.0 & 210.0 & 30.0 & 25.0 \\
\hline 33 & 3.0 & 12.0 & 135.0 & 45.0 & 25.0 \\
\hline 34 & 1.0 & 18.0 & 165.0 & 45.0 & 25.0 \\
\hline 35 & 3.0 & 18.0 & 195.0 & 45.0 & 25.0 \\
\hline 36 & 1.5 & 18.0 & 225.0 & 45.0 & 25.0 \\
\hline 37 & 2.0 & 12.0 & 150.0 & 60.0 & 25.0 \\
\hline 38 & 2.0 & 15.0 & 180.0 & 60.0 & 25.0 \\
\hline 39 & 2.0 & 18.0 & 210.0 & 60.0 & 25.0 \\
\hline
\end{tabular}


Table A.1 - continued from previous page

\begin{tabular}{|c|c|c|c|c|c|}
\hline ID & $\mathbf{d}[\mathbf{m m}]$ & $\mathbf{z}[\mathbf{m m}]$ & $\mathbf{x}[\mathbf{m m}]$ & $\mathbf{y}[\mathbf{m m}]$ & $\mathbf{t}[\mathbf{m m}]$ \\
\hline 40 & 1.0 & 12.0 & 135.0 & 75.0 & 25.0 \\
\hline 41 & 2.0 & 18.0 & 165.0 & 75.0 & 25.0 \\
\hline 42 & 1.5 & 12.0 & 195.0 & 75.0 & 25.0 \\
\hline 43 & 2.5 & 12.0 & 225.0 & 75.0 & 25.0 \\
\hline 44 & 1.0 & 12.0 & 150.0 & 90.0 & 25.0 \\
\hline 45 & 1.0 & 15.0 & 180.0 & 90.0 & 25.0 \\
\hline 46 & 1.0 & 18.0 & 210.0 & 90.0 & 25.0 \\
\hline 47 & 2.0 & 12.0 & 135.0 & 105.0 & 25.0 \\
\hline 48 & 1.0 & 18.0 & 165.0 & 105.0 & 25.0 \\
\hline 49 & 1.0 & 12.0 & 195.0 & 105.0 & 25.0 \\
\hline 50 & 1.5 & 12.0 & 225.0 & 105.0 & 25.0 \\
\hline 51 & 1.0 & 24.0 & 255.0 & 15.0 & 35.0 \\
\hline 52 & 1.5 & 18.0 & 285.0 & 15.0 & 35.0 \\
\hline 53 & 2.5 & 24.0 & 315.0 & 15.0 & 35.0 \\
\hline 54 & 2.5 & 24.0 & 345.0 & 15.0 & 35.0 \\
\hline 55 & 3.0 & 21.0 & 270.0 & 30.0 & 35.0 \\
\hline 56 & 3.0 & 24.0 & 300.0 & 30.0 & 35.0 \\
\hline 57 & 3.0 & 27.0 & 330.0 & 30.0 & 35.0 \\
\hline 58 & 2.0 & 24.0 & 255.0 & 45.0 & 35.0 \\
\hline 59 & 2.0 & 18.0 & 285.0 & 45.0 & 35.0 \\
\hline 60 & 2.5 & 18.0 & 315.0 & 45.0 & 35.0 \\
\hline 61 & 1.5 & 24.0 & 345.0 & 45.0 & 35.0 \\
\hline 62 & 2.0 & 21.0 & 270.0 & 60.0 & 35.0 \\
\hline 63 & 2.0 & 24.0 & 300.0 & 60.0 & 35.0 \\
\hline 64 & 2.0 & 27.0 & 330.0 & 60.0 & 35.0 \\
\hline 65 & 1.0 & 24.0 & 255.0 & 75.0 & 35.0 \\
\hline 66 & 1.5 & 24.0 & 285.0 & 75.0 & 35.0 \\
\hline 67 & 2.5 & 24.0 & 315.0 & 75.0 & 35.0 \\
\hline 68 & 1.5 & 24.0 & 345.0 & 75.0 & 35.0 \\
\hline 69 & 1.0 & 21.0 & 270.0 & 90.0 & 35.0 \\
\hline 70 & 1.0 & 24.0 & 300.0 & 90.0 & 35.0 \\
\hline 71 & 1.0 & 27.0 & 330.0 & 90.0 & 35.0 \\
\hline 72 & 3.0 & 24.0 & 255.0 & 105.0 & 35.0 \\
\hline 73 & 2.0 & 24.0 & 285.0 & 105.0 & 35.0 \\
\hline 74 & 3.0 & 18.0 & 315.0 & 105.0 & 35.0 \\
\hline 75 & 3.0 & 24.0 & 345.0 & 105.0 & 35.0 \\
\hline 76 & 3.0 & 36.0 & 23.0 & 15.0 & 45.0 \\
\hline 77 & 2.5 & 30.0 & 53.0 & 15.0 & 45.0 \\
\hline 78 & 3.0 & 36.0 & 83.0 & 15.0 & 45.0 \\
\hline 79 & 2.0 & 30.0 & 113.0 & 15.0 & 45.0 \\
\hline 80 & 1.0 & 30.0 & 143.0 & 15.0 & 45.0 \\
\hline
\end{tabular}


Table A.1 - continued from previous page

\begin{tabular}{|c|c|c|c|c|c|}
\hline ID & $\mathbf{d}[\mathbf{m m}]$ & $\mathbf{z}[\mathbf{m m}]$ & $\mathbf{x}[\mathbf{m m}]$ & $\mathbf{y}[\mathbf{m m}]$ & $\mathbf{t}[\mathbf{m m}]$ \\
\hline 81 & 2.0 & 30.0 & 38.0 & 30.0 & 45.0 \\
\hline 82 & 1.5 & 36.0 & 68.0 & 30.0 & 45.0 \\
\hline 83 & 1.0 & 36.0 & 98.0 & 30.0 & 45.0 \\
\hline 84 & 2.0 & 30.0 & 128.0 & 30.0 & 45.0 \\
\hline 85 & 1.5 & 30.0 & 23.0 & 45.0 & 45.0 \\
\hline 86 & 3.0 & 30.0 & 53.0 & 45.0 & 45.0 \\
\hline 87 & 2.5 & 30.0 & 83.0 & 45.0 & 45.0 \\
\hline 88 & 1.0 & 30.0 & 113.0 & 45.0 & 45.0 \\
\hline 89 & 1.5 & 36.0 & 143.0 & 45.0 & 45.0 \\
\hline 90 & 2.5 & 36.0 & 38.0 & 60.0 & 45.0 \\
\hline 91 & 2.5 & 30.0 & 68.0 & 60.0 & 45.0 \\
\hline 92 & 2.0 & 36.0 & 98.0 & 60.0 & 45.0 \\
\hline 93 & 1.5 & 30.0 & 128.0 & 60.0 & 45.0 \\
\hline 94 & 1.0 & 36.0 & 23.0 & 75.0 & 45.0 \\
\hline 95 & 2.0 & 36.0 & 53.0 & 75.0 & 45.0 \\
\hline 96 & 1.0 & 36.0 & 83.0 & 75.0 & 45.0 \\
\hline 97 & 3.0 & 36.0 & 113.0 & 75.0 & 45.0 \\
\hline 98 & 1.5 & 30.0 & 38.0 & 90.0 & 45.0 \\
\hline 99 & 2.5 & 36.0 & 68.0 & 90.0 & 45.0 \\
\hline 100 & 2.0 & 36.0 & 98.0 & 90.0 & 45.0 \\
\hline 101 & 1.0 & 30.0 & 128.0 & 90.0 & 45.0 \\
\hline 102 & 3.0 & 30.0 & 23.0 & 105.0 & 45.0 \\
\hline 103 & 2.5 & 36.0 & 53.0 & 105.0 & 45.0 \\
\hline 104 & 1.5 & 36.0 & 83.0 & 105.0 & 45.0 \\
\hline 105 & 3.0 & 30.0 & 113.0 & 105.0 & 45.0 \\
\hline 106 & 2.0 & 48.0 & 173.0 & 15.0 & 55.0 \\
\hline 107 & 2.0 & 48.0 & 203.0 & 15.0 & 55.0 \\
\hline 108 & 1.0 & 42.0 & 233.0 & 15.0 & 55.0 \\
\hline 109 & 2.5 & 48.0 & 263.0 & 15.0 & 55.0 \\
\hline 110 & 1.0 & 48.0 & 293.0 & 15.0 & 55.0 \\
\hline 111 & 1.0 & 42.0 & 188.0 & 30.0 & 55.0 \\
\hline 112 & 1.5 & 42.0 & 218.0 & 30.0 & 55.0 \\
\hline 113 & 1.5 & 42.0 & 248.0 & 30.0 & 55.0 \\
\hline 114 & 2.5 & 42.0 & 278.0 & 30.0 & 55.0 \\
\hline 115 & 1.0 & 42.0 & 173.0 & 45.0 & 55.0 \\
\hline 116 & 2.5 & 48.0 & 203.0 & 45.0 & 55.0 \\
\hline 117 & 2.5 & 42.0 & 233.0 & 45.0 & 55.0 \\
\hline 118 & 3.0 & 42.0 & 263.0 & 45.0 & 55.0 \\
\hline 119 & 2.0 & 42.0 & 293.0 & 45.0 & 55.0 \\
\hline 120 & 1.5 & 48.0 & 188.0 & 60.0 & 55.0 \\
\hline 121 & 3.0 & 48.0 & 218.0 & 60.0 & 55.0 \\
\hline
\end{tabular}


Table A.1 - continued from previous page

\begin{tabular}{|c|c|c|c|c|c|}
\hline ID & $\mathbf{d}[\mathbf{m m}]$ & $\mathbf{z}[\mathbf{m m}]$ & $\mathbf{x}[\mathbf{m m}]$ & $\mathbf{y}[\mathbf{m m}]$ & $\mathbf{t}[\mathbf{m m}]$ \\
\hline 122 & 1.0 & 48.0 & 248.0 & 60.0 & 55.0 \\
\hline 123 & 1.5 & 42.0 & 278.0 & 60.0 & 55.0 \\
\hline 124 & 3.0 & 42.0 & 173.0 & 75.0 & 55.0 \\
\hline 125 & 3.0 & 48.0 & 203.0 & 75.0 & 55.0 \\
\hline 126 & 1.5 & 48.0 & 233.0 & 75.0 & 55.0 \\
\hline 127 & 3.0 & 42.0 & 263.0 & 75.0 & 55.0 \\
\hline 128 & 2.0 & 48.0 & 188.0 & 90.0 & 55.0 \\
\hline 129 & 2.5 & 48.0 & 218.0 & 90.0 & 55.0 \\
\hline 130 & 3.0 & 48.0 & 248.0 & 90.0 & 55.0 \\
\hline 131 & 2.5 & 42.0 & 278.0 & 90.0 & 55.0 \\
\hline 132 & 2.0 & 42.0 & 173.0 & 105.0 & 55.0 \\
\hline 133 & 1.5 & 48.0 & 203.0 & 105.0 & 55.0 \\
\hline 134 & 2.0 & 42.0 & 233.0 & 105.0 & 55.0 \\
\hline 135 & 1.0 & 48.0 & 263.0 & 105.0 & 55.0 \\
\hline 136 & 3.0 & 60.0 & 323.0 & 15.0 & 65.0 \\
\hline 137 & 1.5 & 60.0 & 353.0 & 15.0 & 65.0 \\
\hline 138 & 2.0 & 54.0 & 383.0 & 15.0 & 65.0 \\
\hline 139 & 3.0 & 54.0 & 413.0 & 15.0 & 65.0 \\
\hline 140 & 2.5 & 60.0 & 443.0 & 15.0 & 65.0 \\
\hline 141 & 1.0 & 54.0 & 338.0 & 30.0 & 65.0 \\
\hline 142 & 1.5 & 54.0 & 368.0 & 30.0 & 65.0 \\
\hline 143 & 2.5 & 54.0 & 398.0 & 30.0 & 65.0 \\
\hline 144 & 2.0 & 54.0 & 428.0 & 30.0 & 65.0 \\
\hline 145 & 2.0 & 60.0 & 323.0 & 45.0 & 65.0 \\
\hline 146 & 1.0 & 54.0 & 353.0 & 45.0 & 65.0 \\
\hline 147 & 3.0 & 60.0 & 383.0 & 45.0 & 65.0 \\
\hline 148 & 3.0 & 54.0 & 413.0 & 45.0 & 65.0 \\
\hline 149 & 1.5 & 54.0 & 443.0 & 45.0 & 65.0 \\
\hline 150 & 3.0 & 54.0 & 338.0 & 60.0 & 65.0 \\
\hline 151 & 2.5 & 60.0 & 368.0 & 60.0 & 65.0 \\
\hline 152 & 1.5 & 60.0 & 398.0 & 60.0 & 65.0 \\
\hline 153 & 3.0 & 60.0 & 428.0 & 60.0 & 65.0 \\
\hline 154 & 2.5 & 54.0 & 323.0 & 75.0 & 65.0 \\
\hline 155 & 2.5 & 60.0 & 353.0 & 75.0 & 65.0 \\
\hline 156 & 1.0 & 54.0 & 383.0 & 75.0 & 65.0 \\
\hline 157 & 1.5 & 60.0 & 413.0 & 75.0 & 65.0 \\
\hline 158 & 2.0 & 60.0 & 338.0 & 90.0 & 65.0 \\
\hline 159 & 1.5 & 54.0 & 368.0 & 90.0 & 65.0 \\
\hline 160 & 2.0 & 54.0 & 398.0 & 90.0 & 65.0 \\
\hline 161 & 1.0 & 60.0 & 428.0 & 90.0 & 65.0 \\
\hline 162 & 2.0 & 60.0 & 323.0 & 105.0 & 65.0 \\
\hline
\end{tabular}


Table A.1 - continued from previous page

\begin{tabular}{l|r|r|r|r|r}
$\mathbf{I D}$ & $\mathbf{d}[\mathbf{m m}]$ & $\mathbf{z}[\mathbf{m m}]$ & $\mathbf{x}[\mathbf{m m}]$ & $\mathbf{y}[\mathbf{m m}]$ & $\mathbf{t}[\mathbf{m m}]$ \\
\hline 163 & 1.0 & 60.0 & 353.0 & 105.0 & 65.0 \\
164 & 1.0 & 60.0 & 383.0 & 105.0 & 65.0 \\
165 & 2.5 & 54.0 & 413.0 & 105.0 & 65.0 \\
\hline \hline
\end{tabular}

Table A.2: Measured response amplitudes in copper samples

\begin{tabular}{c|r|r|r|r|r} 
ID & $\begin{array}{r}\text { Backwall } \\
\text { echo }\end{array}$ & $\begin{array}{r}\text { Backwall } \\
\text { echo FBH }\end{array}$ & $\begin{array}{r}\text { Maximum } \\
\text { amplitude }\end{array}$ & Noise & SNR \\
\hline 1 & 280.00 & 195.00 & 64.20 & 6.00 & 10.70 \\
2 & 270.00 & 145.00 & 71.40 & 10.00 & 7.14 \\
3 & 270.00 & 179.00 & 15.40 & 10.00 & 1.54 \\
4 & 270.00 & 148.00 & 74.00 & 10.00 & 7.40 \\
5 & 280.00 & 127.00 & 67.30 & 32.00 & 2.10 \\
6 & 280.00 & 133.00 & 74.90 & 16.00 & 4.68 \\
7 & 280.00 & 158.00 & 66.70 & 7.00 & 9.53 \\
8 & 290.00 & 191.00 & 32.50 & 19.00 & 1.71 \\
9 & 290.00 & 177.00 & 64.40 & 12.00 & 5.37 \\
10 & 280.00 & 196.00 & 32.90 & 12.00 & 2.74 \\
11 & 290.00 & 168.00 & 40.40 & 12.00 & 3.37 \\
12 & 280.00 & 178.00 & 57.20 & 22.00 & 2.60 \\
13 & 270.00 & 169.00 & 44.30 & 11.00 & 4.03 \\
14 & 270.00 & 190.00 & 31.30 & 11.00 & 2.85 \\
15 & 300.00 & 167.00 & 57.40 & 9.00 & 6.38 \\
16 & 290.00 & 210.00 & 26.00 & 9.00 & 2.89 \\
17 & 290.00 & 227.00 & 31.40 & 5.00 & 6.28 \\
18 & 300.00 & 188.00 & 42.50 & 9.00 & 4.72 \\
19 & 290.00 & 185.00 & 12.50 & 12.00 & 1.04 \\
20 & 280.00 & 200.00 & 13.00 & 13.00 & 1.00 \\
21 & 290.00 & 233.00 & 13.00 & 6.00 & 2.17 \\
22 & 290.00 & 223.00 & 22.00 & 5.00 & 4.40 \\
23 & 280.00 & 250.00 & 3.20 & 4.00 & 0.80 \\
24 & 290.00 & 160.00 & 61.00 & 4.00 & 15.25 \\
25 & 290.00 & 216.00 & 48.50 & 4.00 & 12.13 \\
26 & 300.00 & 230.00 & 16.10 & 2.00 & 8.05 \\
27 & 300.00 & 181.00 & 51.60 & 2.00 & 25.80 \\
28 & 300.00 & 153.00 & 48.50 & 2.00 & 24.25 \\
29 & 280.00 & 171.00 & 50.00 & 2.00 & 25.00 \\
30 & 310.00 & 122.00 & 63.30 & 5.00 & 12.66 \\
31 & 310.00 & 138.00 & 62.90 & 5.00 & 12.58 \\
32 & 300.00 & 152.00 & 62.60 & 5.00 & 12.52 \\
33 & 310.00 & 130.00 & 66.30 & 2.00 & 33.15 \\
& & & Continued on next page
\end{tabular}


Table A.2 - continued from previous page

\begin{tabular}{l|r|r|r|r|r} 
ID & $\begin{array}{r}\text { Backwall } \\
\text { echo }\end{array}$ & $\begin{array}{r}\text { Backwall } \\
\text { echo FBH }\end{array}$ & $\begin{array}{r}\text { Maximum } \\
\text { amplitude }\end{array}$ & Noise & SNR \\
\hline 34 & 310.00 & 244.00 & 7.90 & 1.50 & 5.27 \\
35 & 300.00 & 157.00 & 72.50 & 1.50 & 48.33 \\
36 & 300.00 & 222.00 & 17.70 & 1.50 & 11.80 \\
37 & 290.00 & 161.00 & 32.90 & 6.00 & 5.48 \\
38 & 290.00 & 170.00 & 25.30 & 6.00 & 4.22 \\
39 & 290.00 & 187.00 & 29.40 & 6.00 & 4.90 \\
40 & 300.00 & 226.00 & 10.70 & 5.00 & 2.14 \\
41 & 300.00 & 201.00 & 32.10 & 5.00 & 6.42 \\
42 & 300.00 & 188.00 & 19.60 & 5.00 & 3.92 \\
43 & 310.00 & 142.00 & 49.20 & 5.00 & 9.84 \\
44 & 300.00 & 198.00 & 9.60 & 6.00 & 1.60 \\
45 & 290.00 & 205.00 & 7.90 & 6.00 & 1.32 \\
46 & 310.00 & 235.00 & 10.40 & 6.00 & 1.73 \\
47 & 300.00 & 172.00 & 28.40 & 2.00 & 14.20 \\
48 & 290.00 & 236.00 & 10.40 & 2.00 & 5.20 \\
49 & 300.00 & 203.00 & 10.80 & 2.00 & 5.40 \\
50 & 300.00 & 193.00 & 18.10 & 2.00 & 9.05 \\
51 & 270.00 & 190.00 & 10.10 & 3.00 & 3.37 \\
52 & 270.00 & 136.00 & 16.40 & 1.50 & 10.93 \\
53 & 260.00 & 121.00 & 48.40 & 1.50 & 32.27 \\
54 & 270.00 & 116.00 & 56.80 & 1.50 & 37.87 \\
55 & 280.00 & 92.00 & 64.50 & 5.00 & 12.90 \\
56 & 270.00 & 101.00 & 58.80 & 5.00 & 11.76 \\
57 & 260.00 & 116.00 & 59.60 & 5.00 & 11.92 \\
58 & 300.00 & 172.00 & 36.10 & 1.50 & 24.07 \\
59 & 290.00 & 127.00 & 32.10 & 1.00 & 32.10 \\
60 & 280.00 & 95.00 & 53.50 & 1.00 & 53.50 \\
61 & 280.00 & 169.00 & 20.30 & 1.00 & 20.30 \\
62 & 290.00 & 143.00 & 30.20 & 6.00 & 5.03 \\
63 & 280.00 & 154.00 & 29.60 & 5.00 & 5.92 \\
64 & 270.00 & 157.00 & 31.30 & 5.00 & 6.26 \\
65 & 320.00 & 230.00 & 8.60 & 3.00 & 2.87 \\
66 & 310.00 & 192.00 & 21.00 & 3.00 & 7.00 \\
67 & 310.00 & 127.00 & 71.00 & 3.00 & 23.67 \\
68 & 300.00 & 179.00 & 18.20 & 3.00 & 6.07 \\
69 & 320.00 & 201.00 & 6.30 & 5.00 & 1.26 \\
70 & 300.00 & 196.00 & 9.80 & 5.00 & 1.96 \\
71 & 300.00 & 212.00 & 10.40 & 5.00 & 2.08 \\
72 & 310.00 & 125.00 & 72.90 & 1.00 & 72.90 \\
73 & 300.00 & 162.00 & 33.00 & 1.00 & 33.00 \\
& & & \\
& & & & \\
& & & & & \\
& & & & &
\end{tabular}

Continued on next page 
Table A.2 - continued from previous page

\begin{tabular}{c|r|r|r|r|r} 
ID & $\begin{array}{r}\text { Backwall } \\
\text { echo }\end{array}$ & $\begin{array}{r}\text { Backwall } \\
\text { echo FBH }\end{array}$ & $\begin{array}{r}\text { Maximum } \\
\text { amplitude }\end{array}$ & Noise & SNR \\
\hline 74 & 290.00 & 89.00 & 64.60 & 1.50 & 43.07 \\
75 & 300.00 & 119.00 & 67.60 & 1.00 & 67.60 \\
76 & 110.00 & 50.00 & 26.80 & 6.00 & 4.47 \\
77 & 170.00 & 68.00 & 31.80 & 6.00 & 5.30 \\
78 & 220.00 & 102.00 & 52.70 & 6.00 & 8.78 \\
79 & 260.00 & 121.00 & 26.70 & 6.00 & 4.45 \\
80 & 290.00 & 170.00 & 8.50 & 6.00 & 1.42 \\
81 & 130.00 & 61.00 & 16.40 & 6.00 & 2.73 \\
82 & 170.00 & 109.00 & 10.00 & 6.00 & 1.67 \\
83 & 220.00 & 150.00 & 7.30 & 6.00 & 1.22 \\
84 & 250.00 & 112.00 & 27.30 & 6.00 & 4.55 \\
85 & 120.00 & 64.00 & 8.10 & 3.50 & 2.31 \\
86 & 130.00 & 47.00 & 36.70 & 3.50 & 10.49 \\
87 & 150.00 & 58.00 & 29.90 & 3.50 & 8.54 \\
88 & 170.00 & 103.00 & 4.00 & 3.50 & 1.14 \\
89 & 190.00 & 125.00 & 13.80 & 3.50 & 3.94 \\
90 & 120.00 & 60.00 & 22.90 & 6.00 & 3.82 \\
91 & 110.00 & 44.00 & 24.50 & 6.00 & 4.08 \\
92 & 100.00 & 60.00 & 12.80 & 6.00 & 2.13 \\
93 & 100.00 & 54.00 & 10.50 & 6.00 & 1.75 \\
94 & 140.00 & 102.00 & 5.60 & 3.50 & 1.60 \\
95 & 120.00 & 70.00 & 15.70 & 3.50 & 4.49 \\
96 & 110.00 & 78.00 & 3.30 & 3.50 & 0.94 \\
97 & 120.00 & 54.00 & 27.10 & 3.50 & 7.74 \\
98 & 170.00 & 87.00 & 12.80 & 6.00 & 2.13 \\
99 & 160.00 & 78.00 & 32.20 & 6.00 & 5.37 \\
100 & 150.00 & 89.00 & 18.20 & 6.00 & 3.03 \\
101 & 160.00 & 96.00 & 6.00 & 6.00 & 1.00 \\
102 & 210.00 & 68.00 & 58.10 & 1.00 & 58.10 \\
103 & 190.00 & 96.00 & 39.80 & 1.00 & 39.80 \\
104 & 180.00 & 112.00 & 13.60 & 1.00 & 13.60 \\
105 & 180.00 & 61.00 & 43.70 & 1.00 & 43.70 \\
106 & 330.00 & 208.00 & 42.70 & 6.00 & 7.12 \\
107 & 320.00 & 212.00 & 41.00 & 6.00 & 6.83 \\
108 & 300.00 & 216.00 & 10.90 & 6.00 & 1.82 \\
109 & 300.00 & 177.00 & 57.70 & 6.00 & 9.62 \\
110 & 280.00 & 226.00 & 9.60 & 6.00 & 1.60 \\
111 & 290.00 & 205.00 & 8.10 & 5.00 & 1.62 \\
112 & 280.00 & 181.00 & 18.40 & 5.00 & 3.68 \\
113 & 260.00 & 160.00 & 18.10 & 5.00 & 3.62 \\
& & & Continued on next page
\end{tabular}


Table A.2 - continued from previous page

\begin{tabular}{|c|c|c|c|c|c|}
\hline ID & $\begin{array}{c}\text { Backwall } \\
\text { echo }\end{array}$ & $\begin{array}{c}\text { Backwall } \\
\text { echo FBH }\end{array}$ & $\begin{array}{l}\text { Maximum } \\
\text { amplitude }\end{array}$ & Noise & SNR \\
\hline 114 & 230.00 & 117.00 & 46.90 & 5.00 & 9.38 \\
\hline 115 & 220.00 & 147.00 & 5.00 & 3.50 & 1.43 \\
\hline 116 & 220.00 & 132.00 & 39.30 & 3.50 & 11.23 \\
\hline 117 & 220.00 & 107.00 & 39.70 & 3.50 & 11.34 \\
\hline 118 & 210.00 & 89.00 & 48.20 & 3.50 & 13.77 \\
\hline 119 & 190.00 & 101.00 & 21.90 & 3.50 & 6.26 \\
\hline 120 & 110.00 & 79.00 & 9.30 & 5.00 & 1.86 \\
\hline 121 & 110.00 & 60.00 & 21.20 & 5.00 & 4.24 \\
\hline 122 & 110.00 & 77.00 & 7.40 & 5.00 & 1.48 \\
\hline 123 & 100.00 & 62.00 & 10.50 & 5.00 & 2.10 \\
\hline 124 & 130.00 & 57.00 & 32.10 & 1.00 & 32.10 \\
\hline 125 & 130.00 & 72.00 & 30.30 & 1.00 & 30.30 \\
\hline 126 & 130.00 & 97.00 & 7.10 & 1.00 & 7.10 \\
\hline 127 & 130.00 & 54.00 & 33.40 & 1.00 & 33.40 \\
\hline 128 & 160.00 & 114.00 & 19.30 & 5.00 & 3.86 \\
\hline 129 & 160.00 & 99.00 & 28.80 & 5.00 & 5.76 \\
\hline 130 & 150.00 & 85.00 & 34.40 & 5.00 & 6.88 \\
\hline 131 & 140.00 & 83.00 & 30.10 & 5.00 & 6.02 \\
\hline 132 & 150.00 & 90.00 & 21.60 & 1.00 & 21.60 \\
\hline 133 & 140.00 & 106.00 & 9.70 & 1.00 & 9.70 \\
\hline 134 & 140.00 & 76.00 & 15.80 & 1.00 & 15.80 \\
\hline 135 & 130.00 & 101.00 & 1.00 & 1.00 & 1.00 \\
\hline 136 & 220.00 & 150.00 & 44.70 & 6.00 & 7.45 \\
\hline 137 & 190.00 & 152.00 & 13.60 & 6.00 & 2.27 \\
\hline 138 & 160.00 & 105.00 & 16.10 & 6.00 & 2.68 \\
\hline 139 & 140.00 & 73.00 & 28.40 & 6.00 & 4.73 \\
\hline 140 & 120.00 & 78.00 & 12.40 & 6.00 & 2.07 \\
\hline 141 & 160.00 & 125.00 & 8.10 & 4.00 & 2.03 \\
\hline 142 & 130.00 & 98.00 & 6.30 & 4.00 & 1.58 \\
\hline 143 & 100.00 & 61.00 & 17.70 & 4.00 & 4.43 \\
\hline 144 & 90.00 & 54.00 & 6.50 & 4.00 & 1.63 \\
\hline 145 & 140.00 & 108.00 & 14.70 & 3.00 & 4.90 \\
\hline 146 & 130.00 & 98.00 & 6.20 & 3.00 & 2.07 \\
\hline 147 & 110.00 & 69.00 & 18.80 & 3.00 & 6.27 \\
\hline 148 & 90.00 & 57.00 & 19.10 & 3.00 & 6.37 \\
\hline 149 & 90.00 & 62.00 & 5.10 & 3.00 & 1.70 \\
\hline 150 & 80.00 & 44.00 & 13.40 & 5.00 & 2.68 \\
\hline 151 & 90.00 & 70.00 & 9.80 & 5.00 & 1.96 \\
\hline 152 & 90.00 & 75.00 & 5.50 & 5.00 & 1.10 \\
\hline 153 & 90.00 & 59.00 & 13.40 & 5.00 & 2.68 \\
\hline
\end{tabular}

Continued on next page 
Table A.2 - continued from previous page

\begin{tabular}{r|r|r|r|r|r} 
ID & $\begin{array}{c}\text { Backwall } \\
\text { echo }\end{array}$ & $\begin{array}{r}\text { Backwall } \\
\text { echo FBH }\end{array}$ & $\begin{array}{c}\text { Maximum } \\
\text { amplitude }\end{array}$ & Noise & SNR \\
\hline 154 & 100.00 & 49.00 & 17.80 & 1.50 & 11.87 \\
155 & 90.00 & 70.00 & 11.10 & 1.50 & 7.40 \\
156 & 90.00 & 66.00 & 1.50 & 1.50 & 1.00 \\
157 & 80.00 & 66.00 & 3.20 & 1.50 & 2.13 \\
158 & 100.00 & 81.00 & 9.30 & 4.00 & 2.33 \\
159 & 100.00 & 75.00 & 3.60 & 4.00 & 0.90 \\
160 & 100.00 & 66.00 & 8.90 & 4.00 & 2.23 \\
161 & 90.00 & 79.00 & 3.60 & 4.00 & 0.90 \\
162 & 110.00 & 69.00 & 10.50 & 2.00 & 5.25 \\
163 & 110.00 & 97.00 & 1.30 & 2.00 & 0.65 \\
164 & 110.00 & 94.00 & 1.20 & 2.00 & 0.60 \\
165 & 110.00 & 69.00 & 23.00 & 2.00 & 11.50 \\
\hline \hline
\end{tabular}




\section{Appendix B}

\section{Cast iron specimen}

In this appendix, a cast iron specimen KLM162 used for the experiment with ultrasonic TRL probe is described. Technical drawings of the specimen are given in figure B.1 and figure B.2. Recorded response signal amplitudes from semi-elliptical flaws with the TRL probe are given in table B.1. In the first column an ID number of the flaw is provided (compare with drawings). In the second and the third columns the length of the flaw (length of the axis of the ellipse) and depth extension of the flaw (half the length of the axis) are given respectively. The last column displays measured signal-to-noise ratios. 


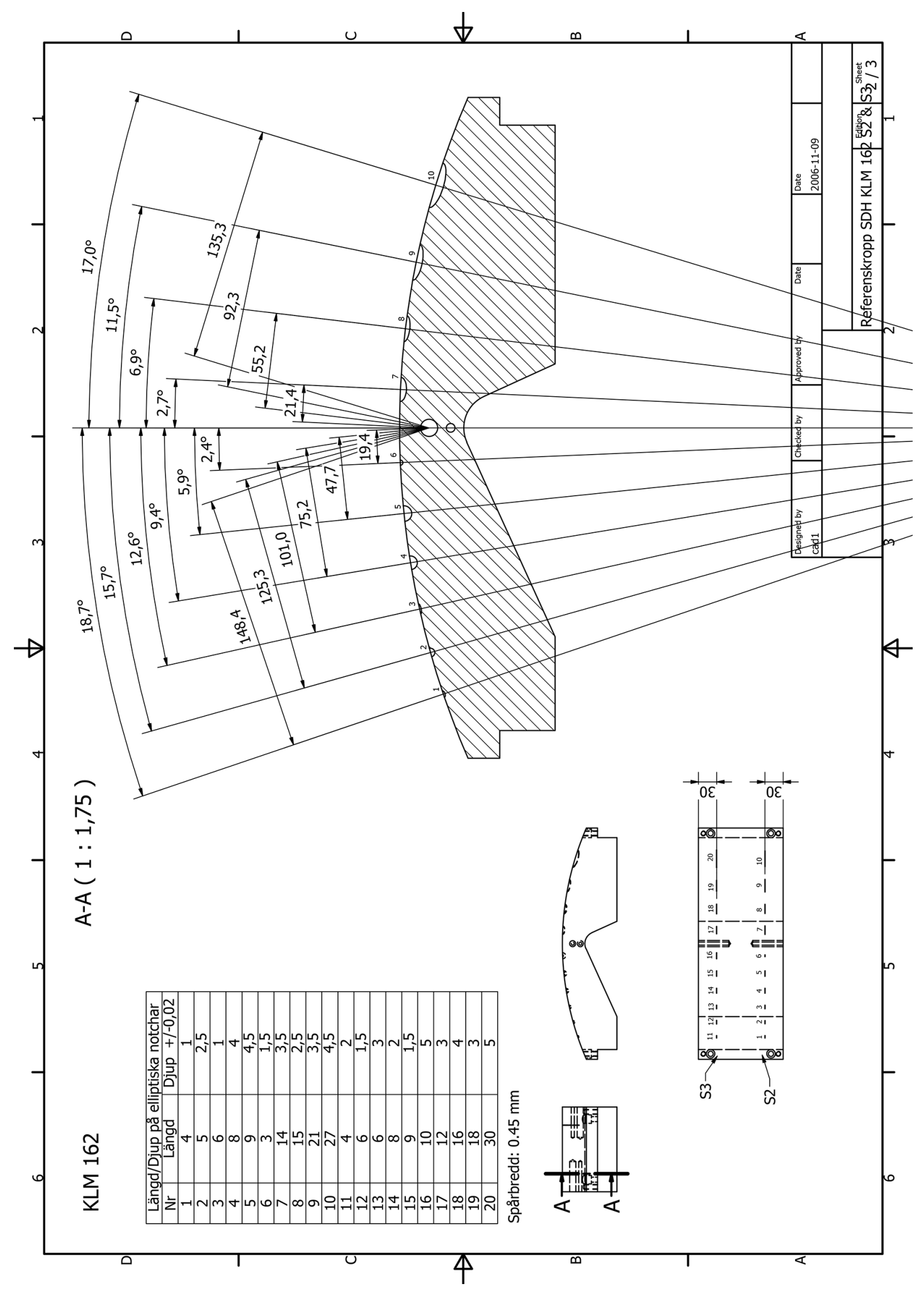

Figure B.1: Cast iron specimen KLM162, section A-A - Iron cast specimen KLM162 with semi-elliptical flaws in section A-A 


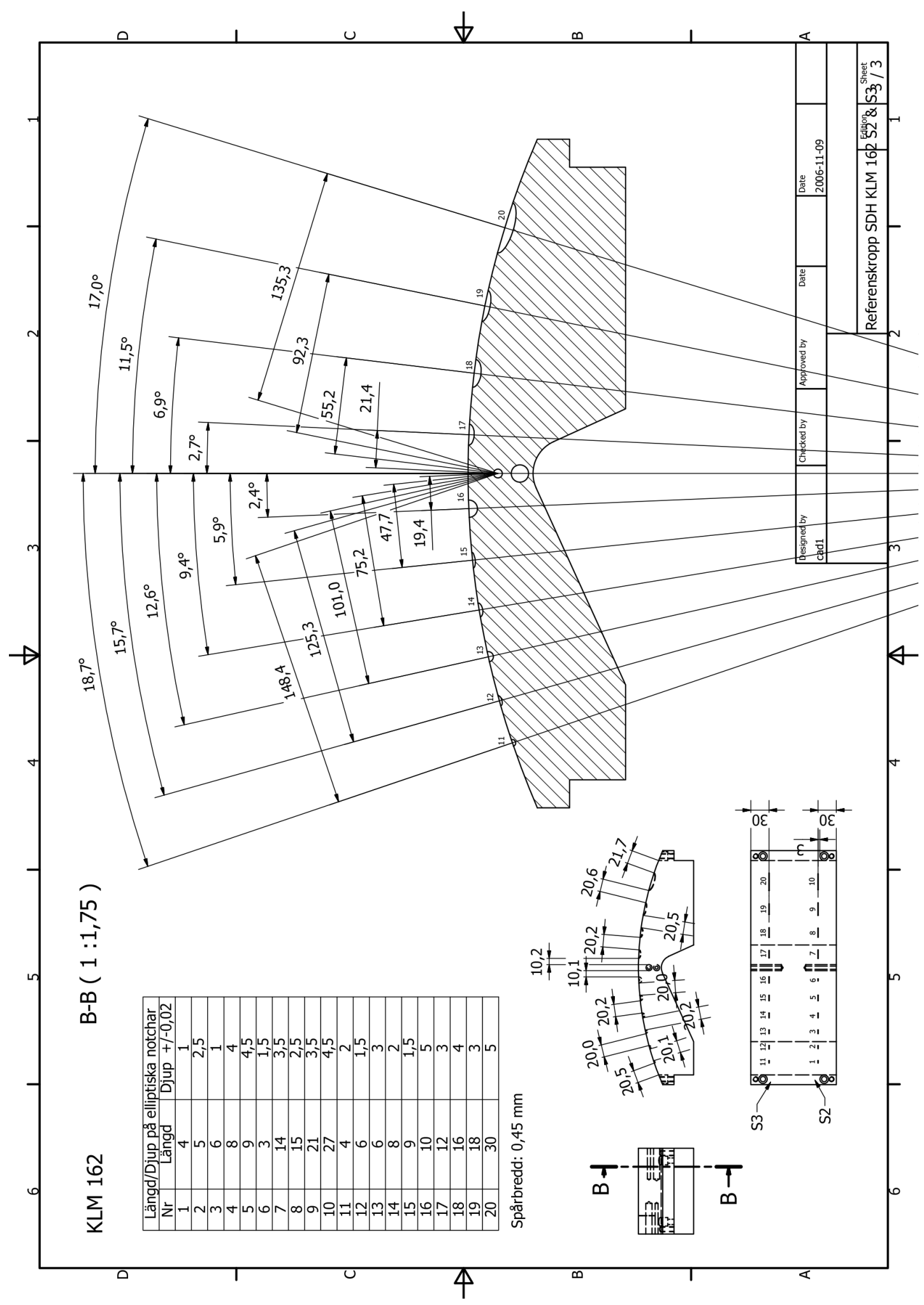

Figure B.2: Cast iron specimen KLM162, section B-B - Iron cast specimen KLM162 with semi-elliptical flaws in section B-B 
Table B.1: Response signal amplitudes, TRL

\begin{tabular}{crrr} 
ID & $\mathbf{l}[\mathbf{m m}]$ & $\mathbf{d}[\mathbf{m m}]$ & $\mathbf{S N R}$ \\
\hline 1 & 4 & 1 & 1.0533 \\
2 & 5 & 2.5 & 3.1500 \\
3 & 6 & 1 & 1.8833 \\
4 & 8 & 4 & 7.3281 \\
5 & 9 & 4.5 & 8.2976 \\
6 & 3 & 1.5 & 1.2133 \\
7 & 14 & 3.5 & 9.3940 \\
8 & 15 & 2.5 & 5.4841 \\
9 & 21 & 3.5 & 7.5014 \\
10 & 27 & 4.5 & 8.8121 \\
11 & 4 & 2 & 2.3233 \\
12 & 6 & 1.5 & 2.1533 \\
13 & 6 & 3 & 5.5423 \\
14 & 8 & 2 & 4.7880 \\
15 & 9 & 1.5 & 3.0100 \\
16 & 10 & 5 & 10.7859 \\
17 & 12 & 3 & 7.7450 \\
18 & 16 & 4 & 8.5867 \\
19 & 18 & 3 & 5.8802 \\
20 & 30 & 5 & 7.1042
\end{tabular}




\section{References}

[1] M. Neil James. Engineering Materialism and Structural Integrity. Journal of Engineering Design, 3:329342, 1998. 1

[2] ASTM E1316 - 07B. Standard Terminology for Nondestructive Examinations. 2007. 1,13

[3] Christina Mueller, Matt Golis, and Tom Taylor. Basic Ideas of the American-European Workshops 1997 in Berlin and 1999 in Boulder. Proceedings of the 15th World Conference on Non-Destructive Testing, Rome, 2000. 126

[4] Ward D. Rummel. Probability of Detection As a Quantitative Measure of Nondestructive Testing End-To-End Process Capabilities. Materials Evaluation, 56, 1998. 2

[5] MIL-HDBK-1823A. Nondestructive Evaluation System Reliability Assessment. DoD, 2009. 227

[6] Luca Gandossi and Charles Annis. ENIQ TGR Technical Document - Probability of Detection Curves: Statistical Best-Practices, ENIQ Report No 41. Technical report, Publications Office of the European Union, 2010. 234

[7] R.H. Burkel, C.-P. Chiou, T.K. Keyes, W.Q. Meeker, J.H. Rose, D.J. Sturges, R.B. Thompson, and W.T. Tucker. A Methodology for the Assessment of the Capability of Inspection Systems for Detection of Subsurface Flaws in Aircraft Turbine Engine Components. Technical report, U.S. Depatment of Transportation, Federal Aviation Administration, 2002. 2

[8] IAEA. Scientific and Technical Basis for Geological Disposal of Radioactive Wastes (IAEA Technical Reports Series No 413). Technical report, International Atomic Energy Agency, 2003. 2015

[9] SKBF/KBS. Final Storage of Spent Nuclear Fuel KBS-3, Summary. Technical report, SKBF/KBS, 1983. 216

[10] Posiva Oy. Safety Case for the Disposal of Spent Nuclear Fuel at Olkiluoto. Technical report, Posiva Oy, 2012. 2

[11] William D. Callister. Materials Science and Engineering. John Wiley and Sons, 2007. 3

[12] Wolfgang W. Seidel and Frank Hahn. Werkstofftechnik. Carl Hanser Verlag München, 2010. 4
[13] Åsa Martinsson, Henrik C.M. Andersson-兰stling, FACredin Seitisleam, Rui Wu, and Rolf Sandström. Creep testing of nodular iron at ambient and elevated temperatures. Technical report, SKB, 2010. 4

14] Claes-Göran Andersson, Peter Eriksson, Westman MARIKa, and Göran EMILsSon. Status report, canister fabrication. Technical report, SKB, 2004. 4

[15] Charles Hellier. Handbook of Nondestructive Evaluation. McGraw-Hill, 2003. 46 6

[16] T.L. Anderson. Fracture Mechanics, Fundamentals and Applications. CRC Press, 1995. 5

[17] I. Milne, R.O. Ritchie, and B. Karihaloo, editors. Comprehensive Structural Integrity: Fracture of Materials from Nano to Macro. Elsevier Science Ltd, 2003. 5

[18] R.B. Thompson. The role of model based inversion Materials Evaluation, 66:707-712, 2008. 6

[19] George A. Matzkanin and H. Thomas Yolken. Probability of Detection (POD) for Nondestructive Evaluation (NDE). Technical report, Nondestructive Testing Information Analysis Center, 2001. 6

[20] Peter J Shull. Nondestructive Evaluation Theory, Techniques, and Applications. Marcel Dekker, Inc., 2002. 7 8

[21] Frederick J Keller, W Edwards Gettys, and Malcolm J Skove. Physics. McGraw-Hill, 1993. 7

[22] Michael Moles and Eric Sjerve. Phased Array IIW Handbook. DVS Media, 2012. 8

[23] Noel Dube, editor. Introduction to Phased Array Ultrasonic Technology Applications. R/D Tech inc., 2004. 8 1277

[24] A. ERHARD. Verfahren der Zerstörungsfreien Materialprüfung, Grundlagen. 2009. 8

[25] Josef Krautkrämer and Herbert Krautkrämer. Werkstoffprüfung mit Ultraschall. Springer-Verlag, 1966. 9 1012

26] Steve Mahaut, Jean-Louis Godefroit, Olivier Roy, and Grard Cattiaux. Application of phased array techniques to coarse grain components inspection. $\mathrm{Ul}$ trasonics, 42(1-9):791-796, 2004. 31. 9

[27] Volker Schuster, Michael Lach, and Michael Platte. Die Qual der Wahl: Welcher Prüfkopf für welchen Einsatz? DACH - Jahrestagung 2004 Salzburg, 2004. 9

28] M. Delaide, G. Maes, and D. Verspeelt, Design and Application of Low-Frequency Twin Side-by-Side Phased Array Transducers for Improved UT Capability on Cast Stainless Steel Components. Procedeeings of the 2nd International Conference on NDE in Relation to Structural Integrity for Nuclear and Pressurized Components, New Orleans, 2000. 9

[29] ASNT. Nondestructive Testing Handbook, 3rd edition, Volume 7: Ultrasonic Testing. ASNT, 1991. 10 
[30] Jonathan J. Kaufman, Wei Xu, Alessandro E. Chiabrera, and Robert S. Siffert. Diffraction effects in insertion mode estimation of ultrasonic group velocity. IEEE Trans. on Ultrasonics, Ferroelectrics, and Frequency Control, Vol. 42(2):232-242, 1995. 11

[31] Terence P. Lerch, Raina Cepel, and Steven P. Neal. Attenuation coefficient estimation using experimental diffraction corrections with multiple interface reflections. Ultrasonics, 44:83-92, 2006. 12

[32] IEA. Key World Energy Statistics 2011. Technical report, International Energy Agency, 2011. 14

[33] Nuclear Energy Institute. Nuclear Energy Around the World 2009. 14

[34] Mazen M. Abu-Khader. Recent advances in nuclear power: A review. Progress in Nuclear Energy, 51:225235, 2009. 14

[35] Michael MeHnert. Endlagerung radioaktiver Abfälle als nationale Aufgabe. Technical report, Bundesamt für Strahlenschutz, 2005. 14

[36] Norbert T. Rempe. Permanent underground repositories for radioactive waste. Progress in Nuclear Energy, 49:365-374, 2007. 15

[37] Michael R. Greenberg, Bernadette M. West, Karen W. Lowrie, and Henry J. MaYer. The Reporter's Handbook on Nuclear Materials, Energy, and Waste Management. Vanderbilt University Press, 2009. 15

[38] Ian G. MCKinley. The management of long lived nuclear waste. Energy Policy, 20:683-692, 1992. 15

[39] P.L. Airey and M. IVAnovich. Geochemical analogues of high-level radioactive waste repositories. Chemical Geology, 55:203-213, 1986. 15

[40] James F. Baldwin, Trevor P. Martin, and Athena ToCATLIDOU. Uncertainty management in radioactive waste repository site assessment. Fuzzy Sets and Systems, 74:81-91, 1995. 15

[41] SKBF/KBS. Final Storage of Spent Nuclear Fuel KBS-3, General. Technical report, SKBF/KBS, 1983. 16

[42] SKBF/KBS. Final Storage of Spent Nuclear Fuel KBS-3, Geology. Technical report, SKBF/KBS, 1983. 16

[43] SKBF/KBS. Final Storage of Spent Nuclear Fuel KBS-3, Barriers. Technical report, SKBF/KBS, 1983. 16

[44] SKBF/KBS. Final Storage of Spent Nuclear Fuel KBS-3, Safety. Technical report, SKBF/KBS, 1983. 16

[45] SKB. Design, production and initial state of the canister. Technical report, SKB, 2010. 18

[46] SKB. Design, production and initial state of the buffer. Technical report, SKB, 2010. 18
[47] SKB. Design, production and initial state of the backfill and plug in deposition tunnels. Technical report, SKB, 2010. 18

[48] SKB. Design, production and initial state of the closure. Technical report, SKB, 2010. 18

[49] SKB. Design and production of the KBS-3 repository. Technical report, SKB, 2010. 18

[50] SKB. Spent nuclear fuel for disposal in the KBS-3 repository, Technical Report TR-10-13. Technical report, SKB, 2010. 18

[51] Karl-Fredrik Nilsson, Frantisek Lofaj, Martin Burström, and Claes-Göran Andersson. Pressure tests of two KBS-3 canister mock-ups TR-05-18. Technical report, SKB, 2005. 20

[52] Peter Dillström and Tobias Bolinder. Damage tolerance analysis of canister inserts for spent nuclea fuel in the case of an earthquake induced rock shear load TR-10-34. Technical report, SKB, 2010. 204445

[53] W.H. Bowyer. A Study of Defects which Might Arise in the Copper Steel Canister. Technical report, SKI, 1999. 20 2122

[54] H. Wirdelius and Elena Österberg. Study of Defect Characteristics Essential for NDT Testing Methods ET, UT and RT. Technical report, SKI, 2000. 20

[55] W.H. Bowyer. Defects Which Might Occur in the Copper-Iron Canister Classified According to their Likely Effect on Canister Integrity. Technical report, SKI, 2000. 20

[56] Carl-Gustaf Öhnfeldt. Feasibility study of detection of defects in thick welded copper. Technical report, SKBF/KBS, 1983. 20

[57] John R. TAYLor. An Introduction to Error Analysis: The Study of Uncertainties in Physical Measurements. University Science Books, 1996. 25

[58] Duane P. Johnson. Inspection uncertainty: The key element in nondestructive inspection. Nuclear Engineering and Design, 43:219 - 226, 1977. 25

[59] M. G. Silk, A.M. Stoneham, and J. A. G. Temple. The Reliability of Non-destructive Inspection. Adam Hilger, 1987. 25]

[60] R.W. Nichols. A review of work related to defining the reliability of non-destructive testing. Nuclear Engineering and Design, 114:1 - 32, 1989. 26

[61] William Q. Meeker. Statistical Methods for Reliability Data. Wiley-Interscience Publication, 1998. 26

[62] A.E. Green And A.J. Bourne. Reliability Technology. Wiley-Interscience Publication, 1972. 26

[63] Merriam-Webster, editor. Merriam-Webster's Collegiate Dictionary. Merriam-Webster, Inc., 2008. 26

[64] n F Haines, D B Lanston, A J Green, and R Wilson. An assessment of the reliability of ultrasonic inspection methods. Periodic Inspection of Pressurized Components, pages 239-255, 1982. 26 
[65] Christina Mueller, Thomas Fritz, Gerd-Ruediger Tillack, Carsten Bellon, and Martina Scharmach. Theory and application of the modular approach to NDT reliability. Materials Evaluation, 59:871-874, 2001. 27

[66] George A. Georgiou. Probability of Detection (POD) curves: Derivation, applications and limitations. Technical report, Prepared by Jacobi Consulting Limited for the Health and Safety Executive, 2006. 27 36

[67] John Swets and Date Arthur. Evaluation of Diagnostic Systems, Methods from Signal Detection Theory. Academic Press, 1982. 28

[68] Thomas D. Wickens. Elemetary Signal Detection Theory. Oxford University Press, 2002. 28

[69] JR CG ANnIS, DL Simms, and JA Harris. A recommended methodology for quantifying NDE/NDI based on aircraft engine experience. AGARD-LS190, 1993. 34.

70] A.H. Ali, D. S. Balint, J. A. G. Temple, and P. LeevERS. Quantifying and Improving the Reliability of NDE Through Modeling Manual Ultrasonic Inspections. AIP Conference Proceedings, 2010. 34

[71] S.K. Nath, Krishnan Balasubramaniam, C.V. Krishnamurthy, and B.H. Narayana. Reliability Analysis of Time of Flight Diffraction Characterization of Inclined Cracks. Materials Evaluation, pages 342-353, 2009. 34

[72] Alan P. Berens. NDE Reliability Data Analysis. ASM International, 17:689-701, 1989. 34 35 $36 \quad 37$

[73] E Ginzel. Ermolov Sizing Equations Revisited. NDT.net, $\mathbf{7}, 2002$. 34

[74] Michele Carboni and Stefano Cantini. A New Approach for the Definition of Probability of Detection Curves. 10th European Conference on NonDestructive Testing, Moscow, 2010. 34

[75] David S. Forsyth and John C. Aldrin. Build Your Own POD. Proceedings of the 4 th European-American Workshop on the Reliability of NDE, Berlin, Germany, 2009. 36

[76] Morris H. Degroot and Mark J. Schervish. Probability and Statistics. Addison-Wesley, 2002. 36

[77] R C H Cheng and T C Iles. Confidence bands for cumulative distribution functions of continuous random variables. Technometrics, 25:77-86, 1983. 36

[78] R. C. H. Cheng and T. C. Iles. One-sided confidence bands for cumulative distribution functions. Technometrics, 30:155-159, 1988. 36

[79] DAVID A BRUCE. NDT reliability estimation from small samples and in-service experience. Technical report, Defence Evaluation and Research Agency UK, 1998. 36
[80] Charles Annis and Luca Gandossi. ENIQ TGR Technical Document - Influence of Sample Size and Other Factors on Hit/Miss Probability of Detection Curves - ENIQ Report nr. 47. Technical report, European Commission Directorate General Joint Research Centre Institute for Energy Petten, The Netherlands, 2012. 36

[81] Hkan Wirdelius and Alexander Blomquist. The Application of Mathematical Modelling of Ultrasonic NDT in the Qualification Process.pdf. Proceedings of the 7th European Conference on Non-Destructive Testing, 1998. 41

[82] R. Bruce Thompson, L. J. Brasche, D. S. Forsyth, E. Lindgren, P. Swindell, and W. Winfree. Recent Advances in Model-Assisted Proability of Detection. Proceedings of the 4th European-American Workshop on the Reliability of NDE, Berlin, Germany, 2009. 41

[83] M. Wall, F. A. Wedgwood, and S. Burch. Modelling of NDT Reliability (POD) and applying corrections for human factors. Proceedings of the 7th European Conference on Non-Destructive Testing, 1998. 41

[84] Martin SpIES. Transducer-modeling in general transversely isotropic media via point-sourcesynthesis: Theory. Journal of Nondestructive Evaluation, 13:85-99, 1994. 41

[85] Martin Spies and Hans Rieder. Synthetic aperture focusing of ultrasonic inspection data to enhance the probability of detection of defects in strongly attenuating materials. $N D T \& S E$ International, 43:425-431, 2010. 41

[86] Rainer Boehm and Anton Erhard. Simulationsgestützte Entwicklung von Ultraschallprüfköpfen. DACH - Jahrestagung Salzburg, 2004. 41

[87] Mato Pavlovic, Kazunori Takahashi, Christina Mueller, Rainer Boehm, and Ulf Ronneteg. NDT reliability Final report, Reliability in non-destructive testing (NDT) of the canister components. Technical report, SKB, 2008. 42

[88] JAN HeRnelind. Modelling and analysis of canister and buffer for earthquake induced rock shear and glacial load TR-10-34. Technical report, 5 T Engineering $\mathrm{AB}, 2010.44$

[89] S.K. Nath, Krishnan Balasubramaniam, C.V. KrishnaMURTHY, AND B.H. NARAYANA. Reliability assessment of manual ultrasonic time of flight diffraction (TOFD) inspection for complex geometry components. NDT \& E International, 43:152-162, 2010. 48

[90] Xavier E. Gros. NDT Data Fusion. Elsevier, 1997. 48

[91] D. HORN AND W. R. MAYO. NDE reliability gains from combining eddy-current and ultrasonic testing. NDT \& E International, 33:351-362, 2000. 48

[92] Bahador Khaleghi, Alaa Khamis, Fakhreddine O. Karray, AND SAIEDEH N. RazaVi. Multisensor data fusion: A review of the state-of-the-art. Information Fusion, 2011. 48 
[93] Mehmet Simsir and Alpay Ankara. Comparison of two non-destructive inspection techniques on the basis of sensitivity and reliability. Materials $\&$ Design, 28:1433-1439, 2007. 48

[94] Mato Pavlovic, Christina Muller, and Christian Boller. The Volume POD as an Optimisation Tool of Multiple NDT Inspections of Complex Geometries. Proceedings of the 2nd International Symposium on NDT in Aerospace, 2010. 48

[95] European methodology for qualification of nondestructive testing - Third issue - ENIQ Report nr. 31. Technical report, European Commission Directorate General Joint Research Centre Institute for Energy Petten, The Netherlands, 2007. 51

[96] ENIQ Recommended Practice 1 - Influential / Essential Parameters - Issue 2 - ENIQ Report nr. 24. Technical report, European Commission Directorate General Joint Research Centre Institute for Energy Petten, The Netherlands, 2005. 52

[97] ENIQ Recommended Practice 2 - Strategy and Recommended Contents for Technical Justifications Issue 2 - ENIQ Report nr. 39. Technical report, European Commission Directorate General Joint Research Centre Institute for Energy Petten, The Netherlands, 2010. 52

[98] Ward D. Rummel. Recommended Practice for a Demonstration of Nondestructive Evaluation (NDE) Reliability on Aircraft Production parts. Materials Evaluation, 40, 1982. 52

[99] Sascha FeistKonn. Gütebewertung qualitativer Prüfaufgaben in der zerstörungsfreien Prüfung im Bauwesen am Beispiel des Impulsradarverfahrens. PhD thesis, Technischen Universotät Berlin, 2012. 52

[100] R.L. Sмітн. The effect of grain size distribution on the frequency dependence of the ultrasonic attenuation in polycrystalline materials. Ultrasonics, 20:211-214, 1982. 65
[101] L Yang and JA Turner. Attenuation of ultrasonic waves in rolled metals. $J$ Acoust Soc Am, 2004. 65

[102] L. Yang, O.I. Lobkis, AND S.I. Rokhlin. Shape effect of elongated grains on ultrasonic attenuation in polycrystalline materials. Ultrasonics, 51:697-708, 2011. 65

103] ASTM E664 / E664M. Standard Practice for the Measurement of the Apparent Attenuation of Longitudinal Ultrasonic Waves by Immersion Method. 2010. 65

[104] V. Rajendran, N. Palanivelu, and B.K. Chaudhuri. A device for the measurement of ultrasonic velocity and attenuation in solid materials under different thermal conditions. Measurement, 38:248-256, 2005. 65

105] Xinbao Liu, Susumu Takamori, Yoshiaki Osawa, and FuXING YIN. Diffraction correction in the measurement of ultrasonic attenuation. Materials Science and Engineering: A, 442:527-531, 2006. 65

[106] Lei Rao, Lian Bing Zhu, Qi Yao Hu, and Xiao Long Li. The Influence of Pore Defects Distribution Characteristic on Casting Service Performance and Fatigue Life. Advanced Materials Research, 2530:476$478,2012.90$

[107] Kazunori Takahashi, Mato Pavlovic, and Christina MÜLLER. Reliability analysis of ultrasonic nondestructive testing by means of probability of detection of real defects using C-scan data. Proceedings of the 4th European-American Workshop on the Reliability of NDE, Berlin, Germany, 2009, 2011. 91

[108] Mato Pavlovic, Kazunori Takahashi, Christina Mueller, and Jorma Pitknen. The Total Reliability of NDT Methods for Inspection of the EB Weld of the Copper Canister used for a Long-term Storage of Spent Nuclear Fuel. Proceedings of the 7th In ternational Conference on NDE in Relation to Structural Integrity for Nuclear and Pressurized Components, 12-15 May 2009, Yokohama, Japan, 2010. 91 